\title{
Cold atoms in cavity-generated dynamical optical potentials
}

\author{
Helmut Ritsch* \\ Institut für theoretische Physik, \\ Universität Innsbruck, \\ Technikerstr. 25, \\ A-6020 Innsbruck, \\ Austria \\ Peter Domokos ${ }^{\dagger}$ \\ Wigner Research Centre for Physics, \\ Hungarian Academy of Sciences, \\ H-1525 Budapest P.O. Box 49, \\ Hungary \\ Ferdinand Brennecke ${ }^{\ddagger}$ and Tilman Esslinger ${ }^{\S}$ \\ Institute for Quantum Electronics, \\ ETH Zürich, CH-8093 Zürich, \\ Switzerland
}

(Dated: 28. September 2012)

\begin{abstract}
We review state-of-the-art theory and experiment of the motion of cold and ultracold atoms coupled to the radiation field within a high-finesse optical resonator in the dispersive regime of the atom-field interaction with small internal excitation. The optical dipole force on the atoms together with the back-action of atomic motion onto the light field gives rise to a complex nonlinear coupled dynamics. As the resonator constitutes an open driven and damped system, the dynamics is non-conservative and in general enables cooling and confining the motion of polarizable particles. In addition the emitted cavity field allows for real-time monitoring of the particle's position with minimal perturbation up to sub-wavelength accuracy. For many-body systems, the resonator field mediates controllable long-range atom-atom interactions, which set the stage for collective phenomena. Besides correlated motion of distant particles, one finds critical behavior and non-equilibrium phase transitions between states of different atomic order in conjunction with superradiant light scattering. Quantum degenerate gases inside optical resonators can be used to emulate opto-mechanics as well as novel quantum phases like supersolids and spin glasses. Non-equilibrium quantum phase transitions as predicted by e.g. the Dicke Hamiltonian can be controlled and explored in real-time via monitoring the cavity field. In combination with optical lattices, the cavity field can be utilized for non-destructive probing Hubbard physics and tailoring long-range interactions for ultracold quantum systems.
\end{abstract}

\section{CONTENTS}

\section{INTRODUCTION}

II. SINGLE ATOMS IN A CAVITY

A. Mechanical effects of light on atoms in a cavity

1. A two-level atom in a cavity

2. Dispersive limit

3. Semiclassical description of atomic motion

B. Cavity cooling

1. Cavity cooling with blue-detuned probe light

2. Cavity cooling and trapping with far red-detuned light

3. Temperature limit

4. Cooling in multimode cavities

\footnotetext{
* helmut.ritsch@uibk.ac.at

† domokos.peter@wigner.mta.hu

¥ brennecke@phys.ethz.ch

$\S$ esslinger@phys.ethz.ch
}

C. Extensions of cavity cooling 12

1. Cooling trapped atoms and ions 12

2. Cooling nanoparticles and relation to optomechanics

3. Cooling molecules

4. Cooling and lasing

5. Monitoring and feedback control

III. COLD ATOMIC ENSEMBLES IN A CAVITY 15

A. Collective coupling to the cavity mode $\quad 15$

1. Cavity-mediated atom-atom interaction $\quad 15$

2. Collective cooling, scaling laws 17

3. Back-action, nonlinear dynamics 18

B. Non-equilibrium phase transitions and collective instabilities

1. Spatial self-organization into a Bragg-crystal

2. Collective atomic recoil laser

C. Phase-space and mean-field descriptions for large particle numbers

1. Critical point

2. Stability analysis and phase diagram

3. Non-equilibrium steady-state distributions 15 (1) 
IV. QUANTUM GASES IN OPTICAL CAVITIES

A. Experimental realizations

B. Theoretical description

C. Cavity opto-mechanics with ultracold atomic ensembles

1. Experimental realizations

2. Nonlinear dynamics and bistability for low photon number

3. Quantum-measurement back-action upon collective atomic motion

4. Cavity cooling in the resolved sideband regime

D. Non-equilibrium phase transitions

1. Self-organization of a Bose-Einstein condensate

2. Open-system realization of the Dicke quantum phase transition

3. Phases in highly degenerate cavities

E. Extended Hubbard-type models for ultracold atoms in cavities

1. Bose-Hubbard model with cavity-mediated atom-atom interactions

2. Cavity-enhanced light scattering for quantum measurement and preparation

3. Self-consistent Bose-Hubbard models in cavity mean-field approximation

\section{OUTLOOK}

References

\section{INTRODUCTION}

Laser light is a versatile tool to cool, prepare and manipulate atoms. Laser cooling (Chu, 1998; Cohen Tannoudji, 1998; Phillips, 1998) and optical pumping (Happer, 1972) relies on spontaneous emission, which is particularly important if the laser frequency is tuned close to the energy of an atomic transition. It is suppressed if the laser frequency is tuned far from any internal excited atomic state. In this limit coherent scattering of photons dominates and the resulting light force, the dipole force, can be derived from an optical potential proportional to the laser intensity inducing a Stark-shift. This forms the basis for trapping and the manipulation of cold atoms (Grimm et al., 2000), Bose-Einstein condensates (Cornell and Wieman, 2002; Ketterle, 2002), quantum gases (Bloch et al., 2008; Giorgini et al., 2008) and mesoscopic particles (Gordon and Ashkin, 1980), where spontaneous emission has to be avoided. In free space the back-action of the particles onto the trapping laser is negligible. In a microscopic picture, this means that the probability of a photon to be scattered by a particle is so small, that the chance for a second scattering event involving the same photon is negligibly small. Hence, the modifications of the field are not felt by the particles and the light forms a conservative optical potential.

The situation changes drastically when the light field is confined in a high-quality optical resonator. Due to multiple round trips of intracavity photons not only the dipole force gets strongly enhanced, but also the backaction of the atoms on the light gets significant. Since atomic motion and cavity field dynamics influence each other, they have to be treated on equal footing. In most cases the dipole force then can no longer be derived from a conservative potential (Horak et al., 1997) and the field dynamics get nonlinear (Vukics et al., 2009).

To get an intuitive picture, consider for example a moving point-like atom, or an entire atomic cloud, forming a dielectric medium with refractive index inside a cavity. This induces a phase shift on the light field that depends on the position and shape of the medium relative to the resonator mode structure. Correspondingly, the cavity resonance frequency is dynamically shifted with respect to the empty cavity. If this shift is comparable to the cavity linewidth, the cavity field intensity, induced by an external pump laser, can undergo a resonant enhancement and so can the back-action on the motion of the medium. For several atoms this coupled atom-field dynamics has the character of a long-range inter-particle interaction. It also generates a strong nonlinear field response, even if the particles are linearly polarizable, as e.g. atoms in the low saturation regime. Coupling to further light modes gives rise to interference effects, which are the origin of collective instabilities and self-organization phenomena. Photons leaking out of the cavity cause a damping of this coupled dynamics. This designable decay channel can be utilized to cool the motion of the medium independent of its specific characteristics.

Historically, cavity quantum electrodynamics (QED) was born as a research field devoted to studying the radiation properties of atoms when boundaries are present (Berman, 1994; Haroche, 1992; Purcell, 1946). Advances in cavity technology over more than 30 years allowed to reach, both in the microwave (Raimond et al., 2001; Walther, 2002) and optical (Kimble, 1998; Mabuchi and Doherty, 2002) frequency domains, the strong coupling regime where the coherent interaction between an atomic transition and a single radiation field mode dominates over all dissipation processes. As a next step, cold and slow atoms have been integrated successfully within optical cavity QED experiments, which led to significant coupling of the atomic motion to the cavity field. It got possible to generate sufficiently strong forces in order to trap an atom in the field of a single photon (Hood et al., 2000; Pinkse et al., 2000). Several experiments achieved strong coupling even in the dispersive regime of cavity QED where the detuning between the light field and the internal atomic transitions is large. Although the resonant energy exchange between atom and field is suppressed in this regime, the position-dependent cavity frequency shift exceeds the cavity linewidth. Motioninduced changes of the effective resonator frequency and its back-action on mechanical motion is also the physical ground of cavity optomechanics (Kippenberg and Vahala, 2008), which can be considered as an extension of dispersive cavity QED towards macroscopic objects.

In this review we survey the recent advancements of cavity QED systems in which coherent momentum ex- 
change between particles and radiation field is the dominating effect of the light-matter interaction. The external degree of freedom of the material component ranges from the center-of-mass motion of a single atom, or a cloud of cold atoms, to the density distribution of a continuous medium such as the quantized matter-wave field of an ultracold gas.

The review illuminates different generic features of the cavity-generated optical dipole force and is structured in three main sections. Briefly summarizing, in Sect. II we discuss the consequences of the retardation between atomic motion and the cavity field dynamics. This time delay leads to an irreversible dynamics that can be the basis of cooling schemes, as presented for single atoms in a cavity. Sect. III discusses how the field modification induced by an atom acts back on the motion of other atoms moving within the cavity. This cavity-mediated atom-atom interaction is a source of collective effects in atomic clouds. Finally, in Sect. IV we consider the collective dynamics of an ultracold gas induced by its strong coupling to the cavity field. Owing to the low temperature, the dynamics involves a reduced set of motional degrees of freedom, and the system becomes a realization of various paradigmatic models of quantum many-body physics and quantum optics (Lewenstein et al., 2007).

The most elementary situation that we will discuss is the dispersive atom-field dynamics of a single atom, or polarizable particle, inside a laser-driven high-finesse cavity (Domokos and Ritsch, 2003; Pinkse and Rempe, 2002). The cavity field dynamically responds to the position and velocity of the particle, thereby generating a time-dependent dipole force acting back onto the particle motion. It is the finite response time of the cavity field which gives rise to the velocity dependent component of the force. It can have the character of a friction force shuffling kinetic energy from the particle to the cavity field and dissipating it via the cavity loss channel. (Vuletić and Chu, 2000). This allows for cooling and self-trapping of particles in the cavity field (Maunz et al., 2004; Nussmann et al., 2005). Sub-recoil cavity cooling of an ultracold atomic cloud has been achieved recently (Wolke et al., 2012), which paves the way towards reaching quantum degeneracy without relying on evaporative cooling techniques. Cavity cooling allows for slowing of any sufficiently polarizable particle with small absorption, without the need of a cycling transition. The possibility of extending the applicability of cavity cooling beyond atoms has been the subject of extensive research in the past years. The light field leaking out the cavity carries information on the trajectory of the particle (Hood et al., 1998; Maunz et al., 2003). Continous monitoring of the atomic motion, in turn, can be used for feedback control (Fischer et al., 2002), which became by today the standard tool to capture single atoms inside a cavity for quantum manipulation (Kubanek et al., 2009).

For cold atomic ensembles inside a laser-driven cavity
(Elsasser et al., 2004; Kruse et al., 2003b) the atom-field coupling strength increases and the dynamics becomes more complex. In many cases the effective coupling strength between particles and cavity field scales with the square root of the particle number (Raizen et al., 1989; Sauer et al., 2004; Tavis and Cummings, 1968; Tuchman et al., 2006). As a consequence of this, the cooling of the center-of-mass motion is correspondingly more effective. Additional complexity arises from the relative motion of the particles, as the local intensity of the cavity field experienced by one atom depends on the position of all other atoms (Horak and Ritsch, 2001c). This gives rise to an effective long-range (Münstermann et al., 2000), or global atom-atom interaction, described by an overall dispersive shift. The contribution of each particle to this shift depends on the local field intensity, which is proportional to the square of the cavity mode function at the position of the atom. In the low excitation regime this can be captured by a collective potential. Furthermore, dissipative forces acting on the relative motion of the particles have been identified (Chan et al., 2003) and interesting correlations between particles can build up (Asbóth et al., 2004).

The cavity-meditated long-range interactions have a different character when the mode of the driving field is not identical with the cavity mode. In this case, the atoms can be considered as sources for the intracavity field and interference between these sources becomes crucial. Correspondingly, the effective cavity driving strength depends on the position of all atoms within the cavity mode profile and it is the field amplitude rather than its intensity, which mediates the long-range interaction. For the case of a transversally laser-driven atomic ensemble in a linear cavity, the long-range interaction causes a phase transition to a self-organized phase, in which the atoms arrange themselves in a checkerboard pattern, thereby maximizing scattering into the cavity mode (Black et al., 2003; Domokos and Ritsch, 2002). In a unidirectionally driven ring cavity geometry, collective scattering between the two counter-propagating cavity modes results in a collective instability, referred to as collective atomic recoil lasing (Kruse et al., 2003a). Various mean-field type theories can be used to describe the non-equilibrium dynamics and asymptotic behavior of large atomic ensembles, including the derivation of scaling laws characterizing the above described critical phenomena (Asbóth et al., 2005; Grießer et al., 2010).

Coupling ultracold atomic ensembles or Bose-Einstein condensates to the radiation field inside a high-finesse resonator, requires a quantized description of the atomic motion and reduces the number of relevant external degrees of freedom (Brennecke et al., 2007; Colombe et al., 2007; Gupta et al., 2007; Slama et al., 2007a). In the case of a laser-driven cavity, situations can be realized where the cavity field couples dominantly to a single collective motional mode of the atomic ensemble, provid- 
ing a direct analogy to cavity optomechanics (Brennecke et al., 2008; Murch et al., 2008; Stamper-Kurn, 2012). Coupling a laser-driven Bose-Einstein condensate to the vacuum field of a cavity, leads to a quantum phase transition between a superfluid and a self-organized phase (Baumann et al., 2010; Nagy et al., 2008). This provides an open-system realization of the Dicke Hamiltonian and its quantum phase transition (Dicke, 1954; Dimer et al., 2007; Hepp and Lieb, 1973; Nagy et al., 2010). The selforganized state can also be considered as a supersolid resulting from a broken Ising-type symmetry. More complex situations occur in highly degenerate multi-mode cavities (Gopalakrishnan et al., 2009, 2011b; Strack and Sachdev, 2011).

Ultracold gases in optical lattices are one of the most intriguing systems in which the power of atomic and laser physics can be exploited to explore generic phenomena of solid-state physics (Bloch et al., 2008). The Hubbard model describing the dynamics of periodically arranged bosons or fermions can be de facto realized with adjustable parameters and variable dimensionality. When the optical lattice potential is created by the field sustained by an optical high-finesse cavity, the corresponding cavity Hubbard-model predicts exotic new phases of matter (Larson et al., 2008a; Maschler and Ritsch, 2005). In many cases the cavity fields provide for a convenient, built-in real-time observation tool. Analyzing the emitted fields allows for dynamical monitoring of quantum phase transitions with minimum and well controlled measurement back-action (Mekhov et al., 2007c).

\section{SINGLE ATOMS IN A CAVITY}

A central objective of cavity quantum electrodynamics (QED) is the perfect control of light-matter interaction at the single-atom and single-photon level in the regime of strong coupling where atom and cavity field form a single entity. A long lifetime of such an 'atomphoton molecule' requires slow and very cold atoms to ensure long interaction times and precise control of the atomic position. At sufficiently small kinetic energies, however, the light forces induced by even a few intracavity photons influence the atomic trajectory. The first cavity QED experiments with cold atoms have already manifested that the cavity light forces guide or deflect slowly moving atoms. In addition, extra diffusion takes place in cavity-sustained dipole traps which may remove the atom from the interaction volume. Clear signatures of such effects have been observed in transmission spectroscopy experiments (Hood et al., 1998; Mabuchi et al., 1996; Münstermann et al., 1999). Time-resolved detection of the transmitted light signal allowed for the reconstruction of atomic trajectories (Hood et al., 2000; Pinkse et al., 2000). These experiments set the stage to include the atomic center-of-mass degrees of freedom and the optical forces in the cavity QED theory. In the following decade, the theoretical and experimental efforts resulted in an extension of the interaction time from the transit-time range of microseconds to the range of minutes (Figueroa et al., 2011; Kubanek et al., 2011).

\section{A. Mechanical effects of light on atoms in a cavity}

The theoretical description of the coupled atom-field dynamics has been presented in detail by Domokos and Ritsch (2003). Here, we recapitulate the notations and methods. Within the vast field of single-atom cavity QED, we restrict ourselves to the atomic motion in a cavity, in particular, to the important concept of cavity cooling. We review the recent experiments demonstrating cavity cooling of single atoms. It is a manifestation of the time-delayed action of the electric dipole force on atoms within the cavity. The understanding at a singleatom level complements nicely another facet of cavity cooling which we will encounter in the case of many atom systems, where it appears in the form of the imaginary part of the collective excitation spectrum.

\section{A two-level atom in a cavity}

We consider a single two-level atom with transition frequency $\omega_{A}$ coupled to a single mode of the electromagnetic field inside an optical resonator with resonance frequency $\omega_{C}$. These frequencies will be referenced to the frequency $\omega$ of an external pump laser by defining the cavity detuning $\Delta_{C}=\omega-\omega_{C}$ and the atomic detuning $\Delta_{A}=\omega-\omega_{A}$. The two relevant atomic states are the ground state $|g\rangle$ and the excited state $|e\rangle$. We introduce the atomic raising and lowering operators, $\sigma^{\dagger}=|e\rangle\langle g|$ and $\sigma=|g\rangle\langle e|$. The cavity mode variables are the photon creation and annihilation operators, $a^{\dagger}$ and $a$, respectively. In the electric-dipole and the rotating-wave approximations and in a frame rotating at the angular frequency $\omega$, the atom-field coupling is described by

$$
H_{\mathrm{JC}} / \hbar=-\Delta_{C} a^{\dagger} a-\Delta_{A}(\mathbf{r}) \sigma^{\dagger} \sigma+i g\left(\sigma^{\dagger} a f(\mathbf{r})-f^{*}(\mathbf{r}) a^{\dagger} \sigma\right),
$$

which is usually referred to as the Jaynes-Cummings Hamiltonian (Jaynes and Cummings, 1963) and, in the quantum optical context, has been reviewed by Shore and Knight (1993). The emphasis here is on that the position $\mathbf{r}$ of the atom is explicitly taken into account. The spatial dependence of the atomic detuning, $\Delta_{A}(\mathbf{r})=$ $\Delta_{A}-\Delta_{S}(\mathbf{r})$, may account for a differential AC-Stark shift $\Delta_{S}(\mathbf{r})$ which can be induced by auxiliary, far-detuned optical trapping fields. The coupling strength in Eq. (1) is spatially modulated according to the intracavity electric field strength which is proportional to the cavity mode function $f(\mathbf{r})$. For the effects reviewed in this paper, it is sufficient to consider modulations on the op- 
tical wavelength scale, thus writing $f(\mathbf{r})=\cos (k x)$ for a standing-wave mode of a Fabry-Pérot resonator, or $f(\mathbf{r})=e^{ \pm i k x}$ for the running-wave modes sustained by a ring resonator ( $k=\omega / c$ is the optical wavenumber). The maximum coupling strength is given by the singlephoton Rabi frequency, $g=d \sqrt{\hbar \omega_{C} / 2 \epsilon_{0} \mathcal{V}}$, where $d$ is the atomic dipole moment along the cavity mode polarization and $\mathcal{V}=\int d^{3} \mathbf{r}|f(\mathbf{r})|^{2}$ denotes the effective cavity mode volume (the maximum of $|f(\mathbf{r})|$ is set to 1 ). The rotating wave approximation relies on that the characteristic frequencies of $H_{\mathrm{JC}}$ are much smaller than the optical frequency, i.e. , $\left(\left|\Delta_{A}\right|,\left|\Delta_{C}\right|, g \ll \omega\right)$. The atomic centerof-mass (CM) motion is a dynamical component of the system, which is described by the Hamiltonian

$$
H_{\text {mech }}=\frac{\mathbf{p}^{2}}{2 m}+V_{\mathrm{cl}}(\mathbf{r}),
$$

where $m$ is the mass of the atom and the term $V_{\text {cl }}$ can represent an arbitrary external trapping potential. For the case of a far off-resonance optical dipole trap, this term, together with the differential AC-Stark shift $\Delta_{S}(\mathbf{r})$ in Eq. (1), fully describe the effect of the trapping laser. The characteristic frequency of the CM motion is given by the kinetic energy of an atom carrying one unit of photon momentum, $|\mathbf{p}|=\hbar k$. We will use throughout the paper the notion of recoil frequency (Cohen-Tannoudji, 1992), with the notation

$$
\omega_{R} \equiv \frac{\hbar k^{2}}{2 m} .
$$

The system can be excited with a coherent laser field at frequency $\omega$, which either drives the cavity mode with driving amplitude $\eta$, or directly the atomic internal degree of freedom at Rabi frequency $\Omega$, described by

$$
H_{\mathrm{pump}} / \hbar=i \eta\left(a^{\dagger}-a\right)+i \Omega h(\mathbf{r})\left(\sigma^{\dagger}-\sigma\right) .
$$

For the case of pumping the atom with a standing-wave laser field from a transverse direction perpendicular to the cavity axis, the spatial mode function is given by $h(\mathbf{r})=\cos (k z) . \quad H_{\text {pump }}$ is effectively time-independent since we work in the frame rotating at the angular frequency $\omega$ of the monochromatic pump laser.

Cavity QED systems in the optical domain are strongly influenced by dissipative coupling to the vacuum modes of the electromagnetic field environment (thermal photons can be neglected at optical frequencies). Correspondingly, the dynamics of the system is described by a quantum master equation (Carmichael, 2003)

$$
\dot{\rho}=-\frac{i}{\hbar}[H, \rho]+\mathcal{L}_{\text {cav }} \rho+\mathcal{L}_{\text {atom }} \rho
$$

with $H=H_{\mathrm{JC}}+H_{\text {mech }}+H_{\text {pump }}$ and $\rho$ denoting the density operator for the atomic (motional and internal) and cavity degrees of freedom. The dissipative processes are captured by the Liouville operators in Born-Markov approximation

$$
\mathcal{L}_{\text {cav }} \rho=-\kappa\left(a^{\dagger} a \rho+\rho a^{\dagger} a-2 a \rho a^{\dagger}\right)
$$

describing decay of the cavity field at rate $\kappa$, and

$$
\begin{aligned}
\mathcal{L}_{\text {atom }} \rho= & -\gamma\left(\sigma^{\dagger} \sigma \rho+\rho \sigma^{\dagger} \sigma\right. \\
& \left.-2 \int d^{2} \mathbf{u} N(\mathbf{u}) \sigma e^{-i k_{A} \mathbf{u r}} \rho e^{i k_{A} \mathbf{u r}} \sigma^{\dagger}\right)
\end{aligned}
$$

describing spontaneous decay of the excited state $|e\rangle$ at rate $\gamma$ accompanied by the emission of a photon into the free-space modes of the electromagnetic field environment. This process involves a recoil of $k_{A}=\omega_{A} / c \approx k$ opposite to the direction $\mathbf{u}$ of the emitted photon, which is averaged over the directional distribution function $N(\mathbf{u})$ characterizing the given atomic transition.

In general, the full quantum dynamics of the system defined by Eq. (4) including all degrees of freedom - the CM motion, the internal electronic dynamics and the cavity photon field - cannot be solved analytically even for a single atom.

\section{Dispersive limit}

For a broad class of cavity QED parameters, atomic saturation effects are negligible and the atoms can be considered as linearly polarizable particles. This holds true when the internal atomic variables $\sigma, \sigma^{\dagger}$ evolve on a much faster time scale as compared to the other variables due to a large atomic detuning $\Delta_{A}$ or a large spontaneous decay rate $\gamma$. In either case, following the usual technique of adiabatic elimination, the atomic polarization operator $\sigma$ can be 'slaved' to the cavity mode and atomic position 'master' variables. In the absence of direct atom driving, i.e. , $\Omega=0$ in Eq. (3), one obtains

$$
\sigma \approx \frac{g f(\mathbf{r}) a}{-i \Delta_{A}+\gamma}
$$

This approximation is valid if the population in the excited atomic state is negligible (low saturation regime). By inserting the slaved variable $\sigma$ into $H_{\mathrm{JC}}$ and into the Liouville operator Eq. (5b), an effective master equation is obtained. Of particular interest is the large detuning limit in which the CM motion and the cavity mode are coupled dispersively by

$$
H_{\mathrm{eff}}=-\hbar\left(\Delta_{C}-U_{0}|f(\mathbf{r})|^{2}\right) a^{\dagger} a .
$$

It captures, on the one hand, the atom-induced dispersive shift of the cavity mode resonance frequency which depends on the momentary position of the atom. On the other hand, the cavity field gives rise to an optical potential $\propto|f(\mathbf{r})|^{2}$ felt by the atom whose depth depends 
on the dynamical photon number. Dissipation can be treated analogously and the effective Liouville operator was presented by Domokos et al. (2001). The dispersive and absorptive effects of the atom are expressed in terms of the parameters

$$
\begin{aligned}
& U_{0}=\frac{g^{2} \Delta_{A}}{\Delta_{A}^{2}+\gamma^{2}}=-\frac{\omega_{C}}{\mathcal{V}} \chi^{\prime}, \\
& \Gamma_{0}=\frac{g^{2} \gamma}{\Delta_{A}^{2}+\gamma^{2}}=-\frac{\omega_{C}}{\mathcal{V}} \chi^{\prime \prime},
\end{aligned}
$$

respectively. These relations reveal the connection between the cavity QED parameters and the complex susceptibility $\chi=\chi^{\prime}-i \chi^{\prime \prime}$ of a linearly polarizable object with electric polarization $\mathbf{P}=\varepsilon_{0} \chi \mathbf{E}$. With this connection at hand, the theory presented in this section can be used to describe a much broader class of particles than only two-level atoms, and most of the findings can directly be applied to polarizable particles of subwavelength size. In Sect. II.C.3, the linear polarizability picture is refined for the case of molecules.

Using the dispersive interaction Hamiltonian, Eq. (7), the quantized one-dimensional motion of a single atom strongly coupled to single-mode cavity field has been numerically simulated (Vukics et al., 2005). The calculation confirmed the basic assumption of semiclassical theories (see Sec. II.A.3), stating that the coherence length of the atomic wavefunction reduces well below the optical wavelength after a few irreversible scattering events. This happens although in the dispersive limit the coupling to the environment is provided by cavity photon loss rather than spontaneous photon scattering into free space. An efficient numerical code has been developed providing a general framework for Monte-Carlo wavefunction simulations of systems composed of the 'quantum optical toolbox' (Vukics, 2012; Vukics and Ritsch, 2007).

If the atom is laser-driven from a transverse direction, i.e. , $\Omega \neq 0$ in Eq. (3), the adiabatic elimination of the internal degrees of freedom leads to

$$
\sigma \approx \frac{g f(\mathbf{r}) a+\Omega h(\mathbf{r})}{-i \Delta_{A}+\gamma} .
$$

Consequently, additional terms appear in the effective adiabatic Hamiltonian Eq. (7) and the Liouvillean Eq. (5b). In particular, coherent photon scattering between the transverse laser field and the cavity mode gives rise to the effective cavity pump term

$$
H_{\text {pump }} / \hbar=\eta_{\text {eff }} h(\mathbf{r})\left(f^{*}(\mathbf{r}) a^{\dagger}+f(\mathbf{r}) a\right),
$$

with the effective cavity drive amplitude

$$
\eta_{\mathrm{eff}}=\frac{\Delta_{A} g \Omega}{\Delta_{A}^{2}+\gamma^{2}} .
$$

The atomic recoil accompanied by photon scattering is accounted for by the spatial dependence of this term.

\section{Semiclassical description of atomic motion}

In many cavity QED experiments, cold atoms are released from a magneto-optical trap into the resonator volume. As the temperature $T$ of the atoms is well above the recoil temperature, $k_{B} T \gg \hbar \omega_{R}$, where $k_{B}$ is the Boltzmann constant, one can assume that the reduced density matrix is almost diagonal both in position and momentum representation. This allows to treat the position $\mathbf{r}$ and momentum $\mathbf{p}$ of the atom as stochastic c-number variables. The regime of ultracold atoms $k_{B} T \lesssim \hbar \omega_{R}$ will be treated in Sec. IV.

a. Langevin-type equation The separation of the quantized internal and the classical motional degrees of freedom was developed for the description of lasercooling (Cohen-Tannoudji, 1992; Dalibard and CohenTannoudji, 1985; Gordon and Ashkin, 1980). This approach has been adopted to the cavity QED scenario by extending the internal degrees of freedom to the combined space of the atomic polarization and cavity mode (Hechenblaikner et al., 1998; Horak et al., 1997). By eliminating the internal degrees of freedom, the dynamics of the CM variables can be formulated in terms of a stochastic differential equation

$$
\begin{aligned}
\dot{\mathbf{r}} & =\frac{\mathbf{p}}{m} \\
\dot{\mathbf{p}} & =\mathbf{f}+\beta \frac{\mathbf{p}}{m}+\boldsymbol{\Xi},
\end{aligned}
$$

where $\mathbf{f}$ denotes the classical force and $\beta$ a friction coefficient in a non-conservative and velocity dependent force term. In general, $\beta$ can be a tensor in the threedimensional space as atomic motion along any direction gives rise to friction in all three spatial directions (Vukics et al., 2004). When the eigenvalues of the tensor $\beta$ (or scalar in 1D) are negative, one encounters cavity cooling. The noise term $\boldsymbol{\Xi}$ induces the stochastic behavior. It has vanishing mean value and is defined via the diffusion matrix $\mathbf{D}$ according to

$$
\left\langle\boldsymbol{\Xi}(t) \circ \boldsymbol{\Xi}\left(t^{\prime}\right)\right\rangle=\mathbf{D} \delta\left(t-t^{\prime}\right),
$$

where $\circ$ denotes the dyadic product. The exact noise correlation function has a width in the range of the dissipative parameters $\kappa$ and $\gamma$ of the internal dynamics. Therefore, it can be approximated by a Dirac- $\delta$ only on the much slower CM motion time scale set by the inverse of the recoil frequency, $\omega_{R}^{-1} \gg \min \left\{\kappa^{-1}, \gamma^{-1}\right\}$. The method of calculating the $c$-number parameters $\mathbf{f}$, $\beta$, and $\mathbf{D}$ of the Langevin-type equation from the master equation concerning the internal degrees of freedom was presented by Hechenblaikner et al. (1998) for the onedimensional, and by Domokos and Ritsch (2003) for the three-dimensional case. This method accounts for the 
quantum effects of the internal dynamics; hence the full approach is semiclassical.

The practical use of this method is strongly limited: the nonlinear quantum master equation for the internal and cavity degrees of freedom has to be solved numerically and for all atomic positions $\mathbf{r}$. Moreover, the Hilbert space of the photon field has to be truncated at low photon numbers. This approach was adopted by Doherty et al. (2000) and Fischer et al. (2001) to simulate the experiments conducted by Hood et al. (2000) and Pinkse et al. (2000).

Analytical approximations can be obtained for the low atomic saturation regime (Murr, 2003), where the atomic polarization can be replaced by a bosonic operator and hence the internal dynamics is described by linear equations of motion. It is then possible to calculate the friction coefficient $\beta$ and, corresponding to its sign, the cooling versus heating regions can be mapped as a function of the detunings $\Delta_{A}$ and $\Delta_{C}$, as shown for example in Fig. 1.
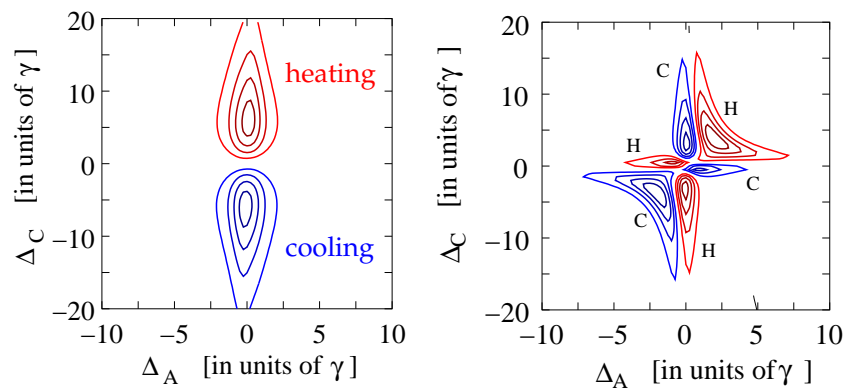

FIG. 1 (Color online) Cooling and heating regions as a function of atom and cavity detunings. Shown are contour plots of the friction coefficient $\beta$ (averaged over an optical wavelength) acting along the cavity axis on a laser-driven atom. Left: Bad-cavity regime, $g=\gamma / 2, \kappa=10 \gamma$; Right: Good cavity regime, $g=3 \gamma, \kappa=\gamma$, where the dressed-state picture can be invoked for interpretation. Blue contour lines indicate cooling $(\mathrm{C}, \beta<0)$, red ones heating $(\mathrm{H}, \beta>0)$ regions. Note that the spatially averaged friction coefficient is shown here; tightly confined atoms localized within a small fraction of the wavelength can follow completely different behavior depending on their position.

b. Semiclassical theory in the dispersive limit In the dispersive limit of atom-cavity coupling presented in Sect. II.A.2, there is an alternative semiclassical approach (Domokos et al., 2001). The Wigner quasi-probability distribution function can be defined in the joint phase space of the atomic CM motion and the cavity field amplitude. The quantum master equation translates then into a partial differential equation for the Wigner function. By dropping all terms containing higher than second-order derivatives, the resulting Fokker-Planck equation corresponds to the evolution of classical stochastic variables associated with the atomic motion and the cavity field. One can consider this approach as the construction of a semiclassical model which lies closest to the true quantum dynamics. As compared to Eq. (11), here large intracavity photon numbers are allowed, in fact, the validity of this approach requires photon numbers larger than 1.

Consider the generic example of a single atom moving in one dimension along the axis of an externally driven linear cavity with the mode function $f(x)=\cos (k x)$, described by the equations (Domokos et al., 2001)

$$
\begin{aligned}
\dot{x} & =\frac{p}{m}, \\
\dot{p} & =-\hbar U_{0}|\alpha|^{2} \frac{\partial}{\partial x} f^{2}(x)+\xi_{p}, \\
\dot{\alpha} & =\eta-i\left(U_{0} f^{2}(x)-\Delta_{C}\right) \alpha \\
& \quad-\left(\kappa+\Gamma_{0} f^{2}(x)\right) \alpha+\xi_{\alpha} .
\end{aligned}
$$

Apart from the noise terms $\xi_{p}$ and $\xi_{\alpha}$, these equations coincide with the classical description in the initial cavity cooling paper by Horak et al. (1997). The force in (13b) acting on the atom is formally identical to the gradient of the optical dipole potential of the cavity mode. The amplitude $\alpha$, however, depends not only on the momentary position of the atom but has a memory effect because of the finite bandwidth $\kappa$ of its linear response. The actual force has therefore a velocity-dependent character which can give rise to a viscous friction force, that is, cavity cooling. Within this approach the friction cannot be determined by a single coefficient $\beta$, on the other hand, the friction effect is correctly described for arbitrary velocity.

The noise sources are taken into account in a consistent way as imposed by quantum mechanics. This results in non-trivial correlations $\left\langle\xi_{p} \xi_{\alpha}\right\rangle \neq 0$. The result for the diffusion matrix as well as the generalization for several atoms was published by Asbóth et al. (2005). The general model is used for numerically studying many-body systems, c.f. Sect. III, well above the temperature of quantum degeneracy.

c. Scattering model The semiclassical Langevin-type equation (11) can be constructed without mode decomposition of the radiation field. This approach is required when, instead of a simple Fabry-Pérot-type cavity geometry, one considers an atom interacting with the radiation field of an interferometer which is composed of an arbitrary one-dimensional configuration of beam splitters. To deal with this situation a scattering model has been established by Xuereb et al. (2009) and solved for the force terms acting on a particle as in Eq. (11) by Xuereb et al. (2010). In the scattering model the atoms and beam splitters are treated on an equal footing as 'scatterers' characterized by a single polarizability parameter. Thereby, a unified framework is created to describe 
opto-mechanical systems in general, which has revealed the close relationship between cavity cooling of atoms and radiation-pressure cooling of mirrors (Arcizet et al., 2006; Gigan et al., 2006; Kleckner and Bouwmeester, 2006; Metzger and Karrai, 2004; Schliesser et al., 2009).

\section{B. Cavity cooling}

One of the most promising results from the understanding of the complex cavity QED dynamics involving atomic motion is the realization of cavity cooling, i.e. , the dissipation of kinetic energy through the cavity photon loss channel in a controlled manner.

Early ideas about using an optical resonator to enhance the efficiency of laser-cooling relied on the modification of the spectral mode density of the electromagnetic radiation field in the presence of spatial boundary conditions (Lewenstein and Roso, 1993; Mossberg et al., 1991). In the most general form, the notion of cavity cooling in the perturbative regime has been expressed by Vuletić and Chu (2000). If an atom, placed inside an optical cavity, is laser driven at a frequency below the cavity resonance, $\Delta_{C}<0$, scattering favors the emission of photons at frequencies higher than the pump frequency due to the increased mode density around the cavity resonance. The energy needed to upshift the photon frequency is provided by the loss in kinetic energy in processes of inelastic scattering. With this very simple picture, a robust three-dimensional cooling effect can be interpreted (Vuletić et al., 2001). However, the picture holds only true in the regime of weak atom-photon coupling, see the left panel of Fig. 1. When the reabsorption of a photon starts to become non-negligible, which happens in a high-finesse cavity, the cooling mechanism substantially changes. This drastic change is illustrated in Fig. 1, where the right panel presents the friction coefficient for a ratio $g / \kappa$ in the the single-atom strongcoupling regime. In the case of a standing-wave cavity, the dynamical cavity cooling effect can be interpreted in the frequency domain by means of a Sisyphus-type argument (Horak et al., 1997), using the dressed-state picture of the strong-coupling regime of cavity QED (Haroche, 1992), see Fig. 2. For the case of a ring cavity, interestingly, the intuitive photon scattering picture can be pursued also in the strong-coupling regime and the full velocity dependence of the radiation pressure for arbitrary coupling constant $g$ can be obtained (Murr, 2006).

In the following we will survey two regimes where cavity cooling has been demonstrated experimentally, and present the corresponding intuitive pictures of the cooling effect. Both regimes are in the dispersive limit of atom-photon interaction keeping the atomic saturation low.

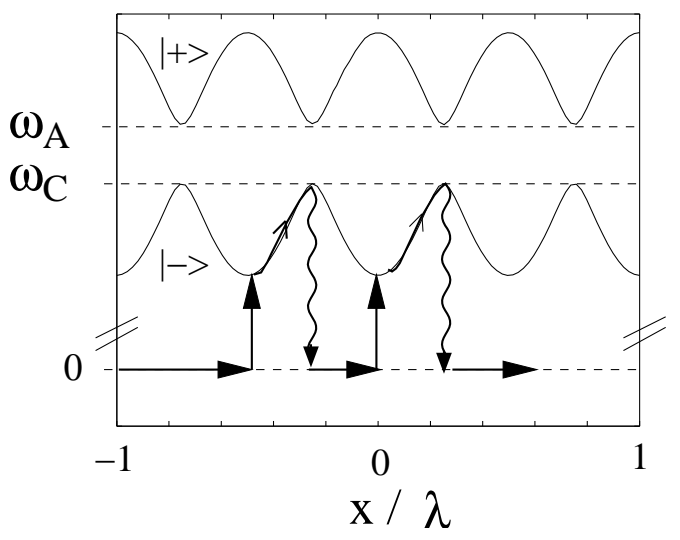

FIG. 2 Sisyphus-type cooling mechanism underlying the hyperbolic-shaped cooling region in the right panel of Fig. 1. The atomic motion leads to a modulation of the internal dressed-state energy levels $| \pm\rangle$ which are linear combinations of the $|g, 1\rangle$ and $|e, 0\rangle$ states with mixing determined by the mode function $f(x)=\cos (k x)$. This amounts to a state-dependent potential for the CM degree of freedom. For $\Delta_{A}<0$ and $\Delta_{C} \approx-\kappa+\frac{g^{2}}{\Delta_{A}}$, the transition from the ground state $|g, 0\rangle$ to the lower dressed state $|-\rangle$ is resonantly excited at an antinode. Thus the excitation happens more likely at the minimum of the potential wells, whereas spontaneous or cavity decay transfers the atom-cavity system back to the ground state homogeneously in space. From Domokos and Ritsch (2003).

\section{Cavity cooling with blue-detuned probe light}

Cavity cooling of single atoms has been first demonstrated by Maunz et al. (2004) via the observation of extended storage times and improved localization of single ${ }^{85} \mathrm{Rb}$ atoms in an intracavity dipole trap. The trap field was red-detuned with respect to the atom, however, the cooling was induced by a weak, blue-detuned probe field. The cooling rate has been estimated to exceed that achieved in free-space cooling methods by at least a factor of five, for comparable excitation of the atom. Maunz et al. (2004) present a very intuitive interpretation of the cooling effect in terms of the classical notion of the refractive index. Consider a standing-wave optical cavity resonantly excited by a weak probe laser, $\Delta_{C}=0$, which is blue-detuned from the atomic resonance by $\Delta_{A}=2 \pi \times 35$ $\mathrm{MHz}>0$, see Fig. 3. The resulting light shift parameter, Eq. (8a), far exceeds the cavity linewidth, $U_{0}>5 \kappa$, so that even one atom can significantly influence the optical path length between the cavity mirrors. Due to Eq. (7), the atom placed at a node of the standing-wave mode profile does not couple to the cavity field and the intracavity intensity is maximum. In contrast, placed at an antinode, the atom shifts the cavity resonance towards higher frequency, i.e. , out of resonance with the probe laser, resulting in a reduced intracavity intensity. In a high-finesse cavity, however, the intensity cannot drop instantaneously when the atom moves away from a node. 
The induced blue-shift of the cavity frequency at almost constant photon number leads to an increase of the energy stored in the field, before the photons are able to leak out of the cavity. This occurs at the expense of kinetic energy of the atoms. The reverse, accelerating effect occurring when the atom moves from an antinode towards a node is much weaker, because the cavity is initially out of resonance with the probe laser and consequently only a small number of photons are present and undergo a corresponding red-shift. This argument also reveals that the delicate correlation between the atomic motion and the photon number variation, underlying the cooling effect, imposes an upper bound on the atomic velocity, $k v<\kappa$, which sets the velocity capture range of cavity cooling.

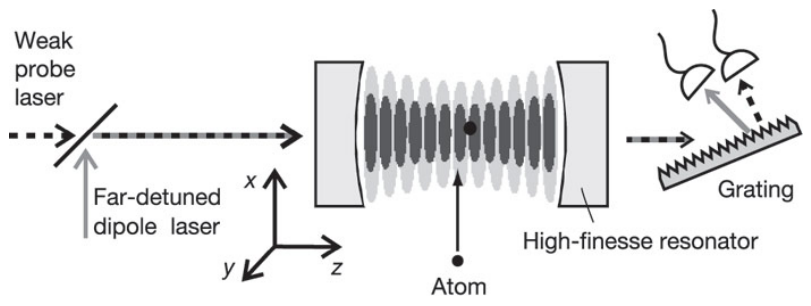

FIG. 3 Experimental scheme used for the observation of cavity cooling. Single atoms are captured by an optical dipole trap formed by far red-detuned light in a longitudinal cavity mode which is different from the one used for cavity cooling. The characteristic parameters of the interaction between the weak probe and the atoms are $(g, \kappa, \gamma)=2 \pi \times(16,1.4,3)$ MHz. From Maunz et al. (2004).

In the experiment, single atoms injected into the cavity are trapped at the field antinodes of a strong intracavity dipole trap. To be detectable in cavity transmission of the weak probe beam, the atoms simultaneously have to be close to an antinode of the probe field mode. As the probe field induces cavity cooling, the resulting stronger confinement can be directly read out of the transmitted signal, as shown in Fig. 4. Time-resolved detection of the cavity transmission allowed to extract a cooling rate of $\beta / m=21 \mathrm{kHz}$, which is large compared to the estimated cooling rate of $4 \mathrm{kHz}$ expected for blue-detuned Sisyphus cooling of a two-level atom in free space, or with the Doppler cooling rate of $1.5 \mathrm{kHz}$ at equivalent atomic saturation.

\section{Cavity cooling and trapping with far red-detuned light}

Far-off-resonance dipole traps are commonly used for long-time capturing and localization of neutral atoms (Grimm et al., 2000). The suppression of spontaneous emission results in an almost conservative trapping potential. However, with the elimination of spontaneous emission $\left(\left|\Delta_{A}\right| \gg \gamma\right)$, any free-space cooling mechanism also disappears. The far-off-resonant trapping scheme

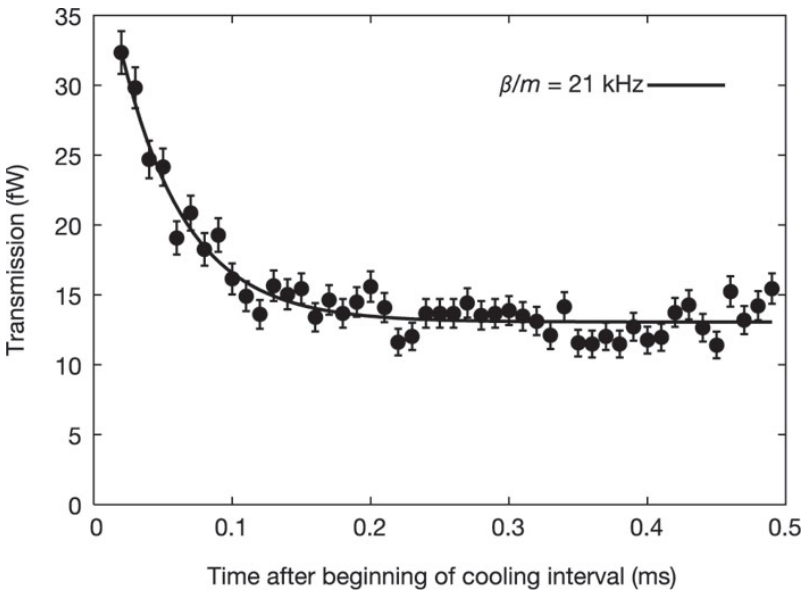

FIG. 4 Demonstration of cavity cooling. Time-resolved reduction of the averaged cavity transmission of a weak resonant probe beam indicating improved localization of the atom at the antinodes of a far red-detuned trapping field. The closer the atom resides at an antinode, the larger the detuning of the cavity resonance with respect to the probe frequency. From Maunz et al. (2004).

has been revisited for a strongly coupled atom-cavity system where the cavity mode provides a new dissipation channel.

Surprisingly, cavity cooling can remain very efficient in the limit of large atomic detuning $\left|\Delta_{A}\right| \rightarrow \infty$. For optimal cooling the driving frequency has to be set slightly below the cavity resonance frequency $\Delta_{C} \approx-\kappa+U_{0}$. Underlying the cooling mechanism is a polariton resonance of the strongly coupled atom-cavity system (corresponding to the dressed state $|-\rangle$ in the weak excitation limit where only the lowest excitations manifold of the Jaynes-Cummings spectrum matters, see Fig. 2). Even if $\omega_{A}$ and $\omega_{C}$ are very different, the bare cavity resonance is slightly modified because the photon excitation mixes with a small amount of the atomic excitation. In an inhomogeneous system, the mixing leads to a dependence of the polariton resonance on the atomic position (see Fig. 2). Although the modulation is tiny in amplitude, the resonance is comparably narrow, having a width in the range of $\kappa$ for the cavity-type polariton. Thus the system can be very sensitive to the atomic motion and even slow atom velocities induce large non-adiabatic modulations of the steady-state field amplitude (Domokos et al., 2004; Murr, 2006).

For demonstration, we consider the simplest case of an atom moving along the cavity axis in the field generated by an external driving laser. It has been shown that the standing-wave cavity field simultaneously traps and cools the atom (Vukics and Domokos, 2005). For a standing- 
wave mode, $f(x)=\cos (k x)$, the cooling rate is given by

$$
\begin{aligned}
& \frac{\beta}{2 \gamma P_{e}}=\frac{\omega_{R}}{\gamma} 4 \sin ^{2}(k x) \times \\
& \times \frac{2 g^{2}\left(\Delta_{C}-U_{0} \cos ^{2}(k x)\right)\left(\kappa+\Gamma_{0} \cos ^{2}(k x)\right)}{\left(\left(\Delta_{C}-U_{0} \cos ^{2}(k x)\right)^{2}+\left(\kappa+\Gamma_{0} \cos ^{2}(k x)\right)^{2}\right)^{2}},
\end{aligned}
$$

where $P_{e}$ denotes the mean population in the excited state $|e\rangle$. Choosing the cavity detuning as $\Delta_{C} \approx-\kappa+U_{0}$ leads to the optimum friction coefficient which, spatially averaged, reads

$$
\frac{\beta}{2 \gamma P_{e}}=\frac{\omega_{R}}{\gamma}\left(\frac{g}{\kappa}\right)^{2}
$$

On the left-hand side the cooling rate is normalized to the rate of spontaneous photon scattering. This expression shows that the friction coefficient at a fixed saturation $P_{e}$ is independent of the atomic detuning, which gives rise to the perspective of cooling molecules or other objects without closed cycling transition (see Sec. II.C.3).

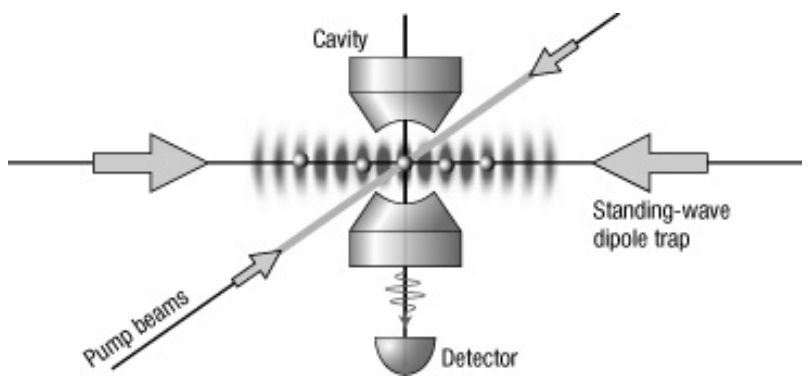

FIG. 5 Transverse pump scheme of cavity QED. Atoms are transported into the cavity using an optical conveyor belt. Instead of driving the cavity directly, the atoms are transversely laser-driven giving rise to photon scattering into the cavity mode. The standing-wave dipole trap yields a large differential AC-Stark shift, i.e. , a modulation of the atomic detuning $\Delta_{A}(\mathbf{r})$, see Eq. (1). In this geometry the cavity vacuum field, the weak driving laser tuned according to the optimum choice in Eq. (15), and the trap laser together form a very efficient three-dimensional cooling scheme. From Nussmann et al. (2005).

Further studies revealed that the previous setting of detunings and intensities can be extended to more general geometries, including the motion perpendicular to the cavity axis, or the external driving of the atom instead of the cavity. What is required for cooling is an inhomogeneity in the system on the wavelength scale which leads to a position-dependent steady-state of the coupled atom-cavity system. This inhomogeneity can arise from the cavity mode function, as for the result in Eq. (14), but also from a standing-wave pump field, or from the spatially modulated AC-Stark shift in a strong standingwave laser field (Murr et al., 2006b). All these sources contribute to the cooling efficiency. The resulting cooling effect has been demonstrated experimentally by Nussmann et al. (2005), making use of an orthogonal arrangement of a cooling laser, a trapping laser and a cavity vacuum mode (see Fig. 5). This combination gives rise to friction forces along all three spatial directions. The achieved cooling efficiency led to microkelvin temperatures and to an average single-atom trapping time in the high-finesse cavity as long as 17 seconds, during which the strongly coupled atom could be observed continuously, see Fig. 6.
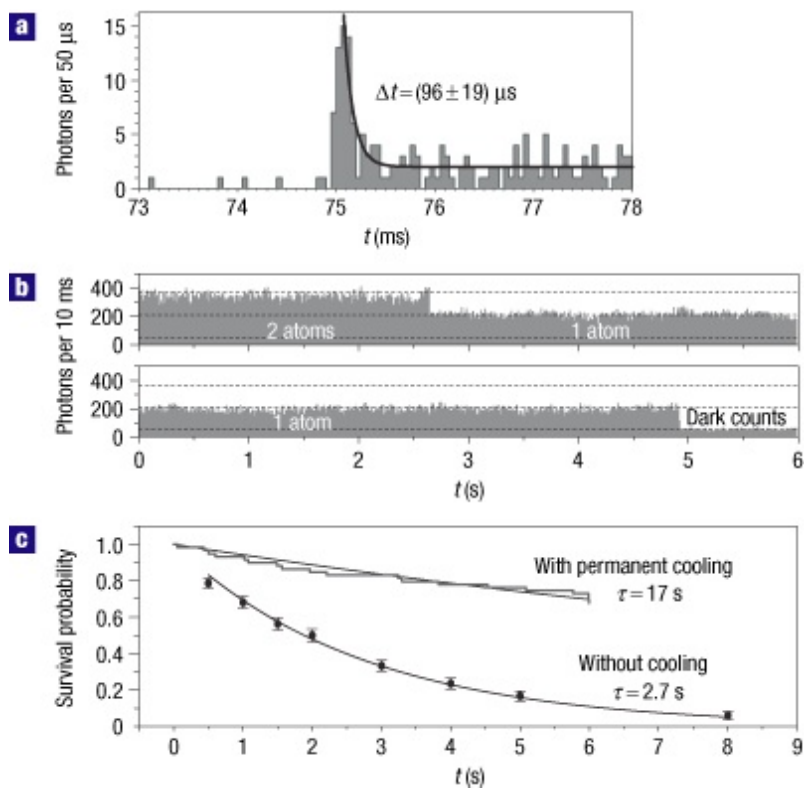

FIG. 6 Demonstration of cavity cooling and long-time trapping of a controlled number of atoms inside the cavity. (a) Shown is a single trace of the recorded photon-count rate indicating the capture of an atom $75 \mathrm{~ms}$ after switching on the pump laser (see Fig. 5). Within $100 \mu \mathrm{s}$, the scattering rate reaches a steady-state value. (b) The recorded photon-count rate allows for determining the atom number and the trapping time. (c) The analysis of 50 traces, each of $6 \mathrm{~s}$ duration and starting with one atom, yields an average lifetime $\tau$ of $17 \mathrm{~s}$ (upper curve), whereas single atoms that are not exposed to the pump laser only reside for $2.7 \mathrm{~s}$ in the cavity volume (lower curve). From Nussmann et al. (2005).

In the experiment (Fig. 5), a far-detuned standingwave dipole trap which is oriented perpendicular to the cavity axis is used to transport atoms into the cavity (Dotsenko et al., 2005; Kuhr et al., 2003). The combination of controlled insertion of single atoms into and retrieval out of a high-finesse optical resonator with cavity cooling led to a deterministic strategy for assembling a permanently bound and strongly coupled atom-cavity system. Long storage times well above 10 seconds and the controlled positioning of single or a given small number of atoms on the submicrometer scale are simultaneously available (Khudaverdyan et al., 2008; Nussmann et al., 
2005).

The exploration of cavity cooling was a stimulating and essential step for the experimental achievement of strongly-coupled cavity QED systems combined with the control over the atomic motion. With the implementation of free-space laser cooling and trapping techniques in cavity experiments sufficiently long atom-cavity interaction times were demonstrated. These achievements led to remarkable experimental breakthroughs and applications in single-atom cavity QED, recently. For example, high-precision measurements demonstrated the basic cavity QED model in the optical domain, i.e. , by resolving the doublet of the lowest-lying excitations of the atom-cavity system Boca et al. (2004) and Maunz et al. (2005), as well as the quantum anharmonic domain of the Jaynes-Cummings spectrum (Kubanek et al., 2008; Schuster et al., 2008) where squeezed light can be readily generated (Ourjoumtsev et al., 2011). Furthermore, the achieved trapping times permitted the development of a deterministic single-photon source (Kuhn et al., 2002; McKeever et al., 2004), for having full polarization control (Wilk et al., 2007b), and to realize the long time sought atom-photon quantum interface (Boozer et al., 2007; Wilk et al., 2007a) and single-atom quantum memory (Specht et al., 2011). Many prosperous directions can grow out from the realization of the elementary case of electromagnetically induced transparency with a single atom (Figueroa et al., 2011; Kampschulte et al., 2010), such as for example all-optical switching with single photons.

\section{Temperature limit}

The extra friction term induced by the cavity is closely connected to the modification of the zero-point field fluctuations. Indeed, even for large atom-field detuning the diffusion within a cavity-sustained optical dipole trap can be an order of magnitude larger than for a free-space field (van Enk et al., 2001; Murr et al., 2006a; Puppe et al., 2007b). The heating rate due to fluctuations can be explicitly calculated in a semiclassical approach (see Sec. II.A.3) which allows to estimate the stationary temperature attained by the atoms. Under optimal conditions one finds the intuitive result

$$
k_{B} T \approx \hbar \kappa,
$$

which is independent of the atomic parameters. This result was confirmed by numerical simulations (Domokos et al., 2001) and fits very well to experimental observations. Interestingly, the result remains largely valid in the limit where the temperature reaches the recoil limit $k_{B} T \approx \hbar \omega_{R}$ which is not governed by the semiclassical description anymore. For a particle trapped in a harmonic potential with vibrational frequency $\nu>\kappa$ (resolved-sideband regime) efficient ground state cooling was proposed by Zippilli and Morigi (2005). Quantum interference effects in the spontaneous emission of a trapped particle in a cavity allow for ground-state cooling even in the bad-cavity regime where $\nu<\kappa$ (Cirac et al., 1995). This prediction does not contradict Eq. (16), since it was made for a strongly localized trapped atom (LambDicke regime) at a precisely given position, whereas the temperature limit above assumes spatial averaging over the cavity wavelength.

According to Eq. (16), there seems to be no lower bound on the temperature as long as the cavity finesse can be increased. However, with decreasing loss rate, the capture range of the cavity cooling mechanism also shrinks. This relation is thoroughly discussed by Murr (2006), based on an explicit expression for the friction force obtained for arbitrary velocity. When applying very strong cavity fields, $\alpha \gg 1$, in close analogy to the mean field treatment of optomechanical models (Genes et al., 2008), it is possible to effectively enhance the weak atomfield interaction appearing at very large detunings to an effective strong coupling $g_{\mathrm{eff}}=g_{0} \alpha$ at the expense of introducing extra fluctuation terms (Nimmrichter et al., 2010). This setting can considerably speed up the cooling process and enhance the capture range, while still leading to a similar final temperature as given by Eq. (16).

\section{Cooling in multimode cavities}

The atom-field dynamics qualitatively changes when invoking several cavity modes to participate as dynamical degrees of freedom. In simple terms, not only the magnitude but also the spatial shape of the optical potential and the associated light forces become a dynamical quantity.

This can be easily demonstrated at the generic example of a ring cavity geometry (Gangl and Ritsch, 2000). In the regime of dispersive atom-field coupling, the atom not only modifies the resonance frequencies of the two counter-propagating cavity modes, thereby tuning their field amplitudes, but also gives rise to phase locking by coherent photon redistribution between the cavity modes. This determines the position of the nodes and antinodes of the emergent standing-wave interference pattern of the cavity radiation field. For a red-detuned pump field, $\Delta_{A}<0$, the particle is drawn to an antinode of the field which, at the same time, gets dragged along with the slowly moving atom (assuming $k v<\kappa, \gamma$ ). Due to the delayed response of the intracavity field, however, the particle is permanently running uphill and thus experiences a friction force. The two-mode geometry of a ring cavity has been shown to result in faster cooling and larger velocity capture range as compared to a singlemode standing-wave cavity (Gangl et al., 2000; Schulze 
et al., 2010). Moreover, the laser pump configuration used for polarization gradient cooling or velocity-selective coherent population trapping can be envisaged within a ring cavity, for which case very efficient cavity cooling is predicted without fundamental lower limit on the temperature (Gangl and Ritsch, 2001)

The more modes in a cavity are available in the vicinity of the pump frequency, the smaller is the transverse length scale on which the field shape gets modulated in the presence of an atom. On the one hand, this leads to stronger three-dimensional localization of atoms around their self-generated intensity maximum (Salzburger et al., 2002). On the other hand, the cooling time reduces in a highly-degenerate confocal cavity more or less quadratically, whereas the diffusion increases only linearly with the effective number of modes involved (Domokos et al., 2002; Nimmrichter et al., 2010).

The scope of cavity-mediated optical manipulation of atoms significantly enlarges also in the case of many-atom systems, which we will review in Sect. IV.D.3.

\section{Extensions of cavity cooling}

The general principle of cavity cooling is expected to be applicable in a broad range of other systems with different radiation field geometries or other material components.

\section{Cooling trapped atoms and ions}

There are several experimental systems in which trapped atoms are strongly coupled to a high-finesse cavity. Ion trap setups have been combined with highfinesse cavities in the moderate coupling regime (Herskind et al., 2009; Keller et al., 2004). There are alloptical schemes, too, where different longitudinal modes of a standing-wave cavity are used to separate the optical trap modes from the cooling ones (Maunz et al., 2004; Schleier-Smith et al., 2011). State-insensitive cooling and trapping of single atoms employing light field at magic wavelengths, which induces an almost identical AC-Stark shift of the two relevant electronic states, has been demonstrated (McKeever et al., 2003). Further, trapping of atoms in low field regions of a blue-detuned intracavity dipole potential has been investigated experimentally (Puppe et al., 2007a). In a similar intracavity dipole trap, the axial atomic motion was cooled down to the ground state by way of coherent Raman transitions on the red vibrational sideband, meanwhile the atomic motion was inferred from the recorded Raman spectrum by Boozer et al. (2006).

The cavity cooling mechanism operates also in the case of tightly confined particles. In the Lamb-Dicke regime for tightly confined particles, $\sqrt{\omega_{R} / \nu} \ll 1$ where $\nu$ de- notes the harmonic trap frequency, explicit expressions for the cooling and heating rates of the CM motion of an atom, trapped in an optical resonator and driven by a laser field, have been derived both in the regime of weak and strong atom-cavity coupling. In the former, a variant of sideband cooling appears (Cirac et al., 1995; Vuletić et al., 2001). Experimentally, the cavity cooling of a single trapped ${ }^{88} \mathrm{Sr}^{+}$ion in the resolved-sideband regime has been demonstrated and quantitatively characterized recently (Leibrandt et al., 2009). The spectrum of cavity transmission, the heating and cooling rates, and the steady-state cooling limit have been measured in perfect agreement with a rate equation theory. The final temperature corresponding to $22.5(3)$ occupied vibrational quanta was limited by the moderate coupling between the ion and the cavity.

The calculations have been extended to the strongcoupling regime, where higher-order transitions between eigenstates of the coupled system have been identified and novel non-trivial parameter regimes leading to cooling have been revealed (Blake et al., 2011; Zippilli and Morigi, 2005). In the resolved-sideband regime, $\nu \gg \kappa, \gamma$, the discreteness of the vibrational spectrum, which is the same for the electronic ground and excited states, gives rise to interference between different transition paths in analogy to the cooling of trapped multilevel atoms (Morigi et al., 2000). Ground state cooling is achievable according to the theoretical predictions (Zippilli and Morigi, 2007).

\section{Cooling nanoparticles and relation to optomechanics}

The fact that cavity cooling requires only linear polarizability suggests that it could be directly applicable to large objects, such as nanobeads (Barker and Shneider, 2010; Chang et al., 2009), thin reflective membranes (Genes et al., 2009), or even small biological objects such as viruses (Romero-Isart et al., 2010). Moreover, since membranes, being macroscopic objects, can have large static polarizability (refractive index), the cooling can be much more efficient than for single atoms or molecules. Indeed, there is a strong connection between cavity cooling of atoms and dispersive cavity optomechanics (Jayich et al., 2008; Thompson et al., 2008), which can easily be seized in the framework of the scattering models (Xuereb et al., 2009). Cavity cooling of membranes experimentally shows great success down to the vibrational quantum ground state (Jayich et al., 2011).

As the local field strength is strongly enhanced inside a resonator, optical dipole traps can be operated at very large detunings, where only the static polarizability of the particle is relevant (Deachapunya et al., 2008; Nimmrichter et al., 2010). In such a setting of coupled optical and mechanical systems, the ring cavity with degenerate pairs of counterpropagating modes, or other configura- 
tions where degenerate modes are available, can offer the realization of various effective models.

Consider, for example, a symmetrically pumped ring cavity. The field can be written as a superposition of the strongly pumped and thus highly excited cosine mode and the empty sine mode. The cosine mode fulfills two purposes: (i) it generates the trapping potential, and (ii) it feeds the sine mode through photon scattering off the particle (atom, molecule, membrane). The model Hamiltonian is of the form (Schulze et al., 2010):

$H=\frac{p^{2}}{2 m}-\hbar \Delta_{C}\left(a_{c}^{\dagger} a_{c}+a_{s}^{\dagger} a_{s}\right)-\hbar U(x)+i \hbar\left(\eta a_{c}^{\dagger}-\eta^{*} a_{c}\right)$,

where $U(x)$ is the dispersive interaction potential, and $a_{c}$ $\left(a_{s}\right)$ denote the field amplitudes of the cosine (sine) mode, respectively. Linearizing the position around the trap minimum, we can recover the standard optomechanical Hamiltonian,

$$
\begin{aligned}
H=\left[\frac{p^{2}}{2 m}\right. & \left.+\frac{1}{2} m 2 \hbar U_{0} a_{c}^{\dagger} a_{c}(k x)^{2}\right] \\
& -\hbar\left(\Delta_{C}-U_{0}\right) a_{c}^{\dagger} a_{c}-\hbar \Delta_{C} a_{s}^{\dagger} a_{s} \\
& -\hbar U_{0}^{\prime}\left(a_{s}+a_{s}^{\dagger}\right) x,
\end{aligned}
$$

with quadratic coupling to the cosine trapping mode and linear coupling to the sine cooling mode. As the particle couples the two modes there appears an energy splitting which allows to extract via inelastic scattering kinetic energy from the vibrational motion in the optical trap (Elsasser et al., 2003). As for standard cavity cooling the final temperature in the classical regime is again limited by the cavity linewidth $k_{B} T \approx \hbar \kappa$. However, in a very good cavity, when the pump field is sufficiently strong, one can reach the resolved-sideband regime, where the trap frequency $\nu$ exceeds the cavity linewidth, and the final temperature would correspond to less than a single excitation $k_{B} T<\hbar \nu$. In this ground-state cooling limit one has to resort to a quantum description of motion and the optical fields. Interestingly, the sine mode automatically acts as a built-in monitoring system which continuously observes the vibrational quantum state of the particle in the cosine mode. Hence close to $T=0$ one can observe quantum jumps of the particle via the sine mode photon counts (Schulze et al., 2010).

\section{Cooling molecules}

a. Cooling the translational motion of molecules Molecular structure fundamentally alters and complicates the picture conceived for laser-cooling two-level atoms. Upon excitation from the pump field, the molecule can relax by either Rayleigh scattering back to the ground state $|g\rangle$ at the rate $\gamma_{\mathrm{Ry}}$ or Raman scattering to metastable states at the rate $\gamma_{\mathrm{Rn}}$. There is a multitude of metastable molecular states (spin-orbit, rotational, and vibrational) available via inelastic Raman scattering. The generally low free-space branching ratio $\gamma_{\mathrm{Ry}} / \gamma_{\mathrm{Rn}}$ results in population shelving after only a few photon scattering events, thereby prematurely quenching the cooling process. Because of the prohibitive expense of building multiple repumping laser systems, optical cooling of molecules via free-space dissipative scattering of photons is thought not to be practicable.

Since cavity-assisted laser-cooling relies on the cavity dissipation channel, it has been suggested as a potential method to mitigate Raman loss. Spontaneous photon scattering, in principle, can be entirely suppressed by using large detuning. However, as discussed in Sec. II.B.2, in order to keep the cooling efficiency constant, one needs to preserve a given level of excitation in the atom or molecule. Therefore, merely the large detuning does not solve the branching ratio problem of molecules (Lev et al., 2008). To overcome this severe problem, the use of an optical cavity with cooperativity parameter much larger than unity is mandatory, in accordance with Eq. (15). In this case the enhanced coherent Rayleigh scattering into a decaying cavity mode can ensure a vanishingly small probability of the molecule to Raman scatter during the cooling time. For CN diatomic molecules, the cavity cooling process has been calculated numerically ( $\mathrm{Lu}$ et al., 2007).

b. Cooling the rotation and vibration of molecules While theoretical models and experiments have mostly concentrated on the center-of-mass motion of structureless polarizable particles or two-level atoms, the complex rovibrational structure of molecules is one of the central obstacles preventing efficient laser-cooling of molecules. In many common beam sources the initial temperature can be designed to be low enough to freeze most vibrations and only leave few rotational quanta (Rangwala et al., 2003). Nevertheless, the interaction with the cooling laser light will in general start to redistribute the population within the rovibrational manifolds strongly altering the optical properties of the molecules and hampering further cooling. Only a few exceptions of this rule have been discovered and investigated lately (Shuman et al., 2010). Cavity cooling, however, can in principle be designed to counteract this heating process and even further cool the rovibrational energy of molecule. As an enourmous spread of transition frequencies is required to facilitate this, it proves advantageous to simultaneously apply a multitude of different longitudinal cavity modes (Kowalewski et al., 2007; Morigi et al., 2007). Simulations show that the rovibrational cooling can be combined with motional cooling, e.g., in a trap (Kowalewski et al., 2011), to get a cold molecular gas in all degrees of freedom. At this point a practical implementation would require precooling by other methods, such as the opto- 
electrical scheme proposed by Zeppenfeld et al. (2009), to achieve sufficient interaction times and densities within the cavity mode volume.

\section{Cooling and lasing}

Collective coherent emission of a laser-driven atomic ensemble into the field of an optical cavity accompanied by a very fast and efficient cooling of atomic motion was observed in an experiment conducted by Chan et al. (2003). Although the effect has not yet been fully understood, it is attributed to Raman gain within a Zeeman manifold. The combination of cavity cooling with intracavity gain is an intriguing prospect. It was initially suggested by Vuletic (2001) to transform a bad cavity effectively into a good cavity with fast cooling towards an even lower temperature. While the principle idea proves to be correct, a more realistic and detailed modeling, which accounts for fluctuations to consistently treat the gain, gives a higher limit of the achievable temperature (Salzburger and Ritsch, 2006). This observation was also confirmed in the optomechanical regime of cavity cooling, where intracavity gain leads to faster cooling but a higher final temperature (Genes et al., 2009).

In a standard setup, the intracavity gain could be generated by an additional inverted medium placed within the cavity. This would lead to a technically challenging setup, if one aims to operate in the strong-coupling regime. Interestingly, it turns out that in a conceptually much simpler configuration, the gain can also be provided by the same atomic medium which is aimed to be cooled in the setup. Of course, such a scheme requires a suitable pumping mechanism which transfers atoms from the lower to the upper level of the cooling transition, without introducing too much extra noise. In the ultimate limit one can envisage a single atom, which is externally pumped within a high-finesse cavity. Stimulated emission into the cavity mode provides gain to create a trapping potential for the atom. For a blue-detuned cavity this gain simultaneously extracts motional energy from the particle and thus provides cooling (Salzburger and Ritsch, 2004). Fortunately, an inverted atom is a high-field seeker in the blue-detuned light field, so that it will be trapped close to optimal gain. Hence this setup provides for lasing, trapping and cooling of a single atom within a resonator forming the most minimalistic implementation of a laser (Salzburger et al., 2005). The system can be generalized to several particles, which strongly reduces the requirements on the pump mechanism (Salzburger and Ritsch, 2006). In the limit of ultracold gases in an optical lattice, stimulated optical gain occurs concurrent with Bose enhanced coherent population of the lowest energy band. While for a pulsed setup this constitutes in principle a very fast and efficient cooling method, a CW setup could provide a possible route towards the realization of a CW atom laser (Salzburger and Ritsch, 2007, 2008).

\section{Monitoring and feedback control}

Starting from the early days of cavity QED, a strongly coupled atom-cavity system was considered as a numberresolving neutral particle detector (Mabuchi et al., 1996), a concept which is still being developed and implemented in miniaturized devices (Teper et al., 2006). Going one step further, the high-finesse resonator acts as a microscope with which the trajectory of individual atoms can be reconstructed from the recorded cavity transmission with high spatial $(<\mu \mathrm{m})$ and temporal $(<\mu \mathrm{s})$ resolution (Hood et al., 2000). The method can be considerably improved by the use of multimode cavities. The particle does not only modify the phase and intensity of the intracavity field, but redistributes light between the different spatial modes. The output field imaged on a CCD camera therefore allows to monitor directly and in real time the motion of the particle (Horak et al., 2002; Maunz et al., 2003). Note that even for incomplete position information at any given time, the most likely trajectory of single atoms can be reconstructed with the help of inversion algorithms based on the coupled equations of motion.

Once the position and motion of the particle are known, it is straightforward to apply feedback on the motion of a single atom by adjusting the pump lasers to steer the particle motion within the cavity and increase its trapping time (Fischer et al., 2002). The cavity field both provides particle detection and mediates the feedback force. This method has been successfully refined by several groups and resulted in an increase of singleparticle trapping times by several orders of magnitude (Kubanek et al., 2009, 2011). By applying controlled and delayed feedback forces on the particle, its kinetic energy can be reduced as well. This kind of feedback cooling resembles stochastic cooling techniques applied in high-energy physics. Strongly enhanced cooling has been predicted when the feedback scheme, consisting of timedependent switching of the trapping field as a function of the intracavity intensity, is operated in the dispersive bistability regime (Vilensky et al., 2007). This method should also give new prospects to optomechanical setups. 


\section{COLD ATOMIC ENSEMBLES IN A CAVITY}

New research directions opened in cavity QED when cold and ultracold atomic ensembles were successfully prepared within high-finesse optical resonators. In the many-body configuration, the common coupling of atoms to the cavity field creates a wealth of new possibilities to implement tailored atom-atom interactions over large distances, an ingredient which usually is absent in freespace cold atom experiments.

The atom-atom coupling is mediated by the cavity radiation field between the $\mathrm{AC}$ electric dipole moments. However, its nature is inherently different from the freespace dipole-dipole interaction. In a cavity, the interaction strength does not decay with the interatomic distance and depends only on the local coupling of the atoms to the cavity field. Fundamentally, the interaction is not binary: the ensemble of atoms collectively acts onto the state of the radiation field which then reacts back on the individual atoms. This scenario is generally referred to as global coupling. The range of the interaction is given by the size of the cavity mode, which can be macroscopic. In cases where single-atom strong coupling is not achieved, the collective energy exchange still can be dominated by coherent interaction.

After discussing the nature of the long range atomatom interaction mediated by a cavity field in various geometries, we consider first the many-body influence on the cavity cooling scheme. Then we address the most spectacular collective effects realized by cold atoms within linear and ring cavities. Critical phenomena, instability thresholds, and scaling laws will be discussed by means of various mean field theories in the end of this section.

\section{A. Collective coupling to the cavity mode}

Resonant coherent coupling between an ensemble of $N$ two-level atoms and a single standing-wave cavity mode is described by the many-body generalization of Eq. (1)

$$
\begin{aligned}
H / \hbar=-\Delta_{C} a^{\dagger} a-\sum_{j} & \Delta_{A}\left(\mathbf{r}_{j}\right) \sigma_{j}^{\dagger} \sigma_{j} \\
& +\sum_{j} i g f\left(\mathbf{r}_{j}\right)\left(\sigma_{j}^{\dagger} a-a^{\dagger} \sigma_{j}\right)
\end{aligned}
$$

where $j=1 \ldots N$ labels the atoms, and the mode function $f(\mathbf{r})$, for simplicity, is real. The atomic ensemble can be represented by a single collective dipole with effective coupling strength only if (i) the atomic motion can be averaged out (ii) only the cavity mode is laser-driven, and (iii) the atoms are in the low saturation regime. In this case the atoms collectively couple to the cavity mode with an effective strength of $g_{\text {eff }}=g \sqrt{\sum_{j} f^{2}\left(\mathbf{r}_{j}\right)}$ (the summation index runs from 1 to $N$ ). Correspondingly, an
$N$-fold enhancement appears for the many-atom system in terms of the single-atom cooperativity, $\mathcal{C}=g^{2} /(2 \kappa \gamma)$, which measures the ratio of light scattering into cavity mode versus surrounding vacuum modes (Tuchman et al., 2006). For example, the strong distortions of the singleatom normal mode splitting in the cavity transmission spectrum induced by a thermal beam of atoms crossing the cavity could be interpreted by such a collective mode picture (Raizen et al., 1989). Employing an optical conveyor belt, an adjustable number, $N=1 \ldots 100$, of cold atoms has been transported into a microcavity, and large nonlinearities have been achieved as evidenced by the observation of absorptive optical bistability in the real-time transmission spectrum (Sauer et al., 2004).

In general, however, one has to consider the manybody system composed of a large number of internal and motional degrees of freedom. We will exhibit this in the following at the simplest nontrivial case of two atoms in the same mode.

\section{Cavity-mediated atom-atom interaction}

Let us discuss the character of the cavity-mediated atom-atom interaction in two different pump geometries, namely, pumping the cavity field either directly or indirectly via light scattering off the laser-driven atoms.

a. Cavity pumping Consider $N$ atoms moving in the field of a laser-driven optical cavity. The detuning between the driving laser and the dispersively shifted cavity resonance depends on the position of all atoms, which in turn experience the optical dipole force of the intracavity field. For small atomic velocities and in the low saturation limit, an adiabatic potential can be deduced (Fischer et al., 2001)

$$
V\left(\mathbf{r}_{1}, \ldots \mathbf{r}_{N}\right)=\frac{\hbar \Delta_{A}|\eta|^{2}}{\Delta_{A} \kappa+\Delta_{C} \gamma} \operatorname{atan} \frac{\gamma \kappa-\Delta_{A} \Delta_{C}+g_{\mathrm{eff}}^{2}}{\Delta_{A} \kappa+\Delta_{C} \gamma},
$$

which is analogous to the Born-Oppenheimer approximation used for describing the motion of nuclei in molecules in the averaged electronic potential. The potential $V$ depends on the atomic positions solely via the collective coupling strength $g_{\text {eff }}$, and thus is valid for any number of atoms. This is not surprising as the adiabatic force is calculated by freezing the atomic motion. The resulting cavity-mediated long-range atom-atom interaction gives rise to an asymmetric deformation of the normal-mode splitting as was observed experimentally by Münstermann et al. (2000).

In the case of two atoms with positions $x_{1}$ and $x_{2}$ the interaction potential landscape $V\left(x_{1}, x_{2}\right)$ along the cavity axis is shown in Fig. 7 for two different parameter settings. The upper graph corresponds to the experimental parameters used in the Garching group (Münstermann 


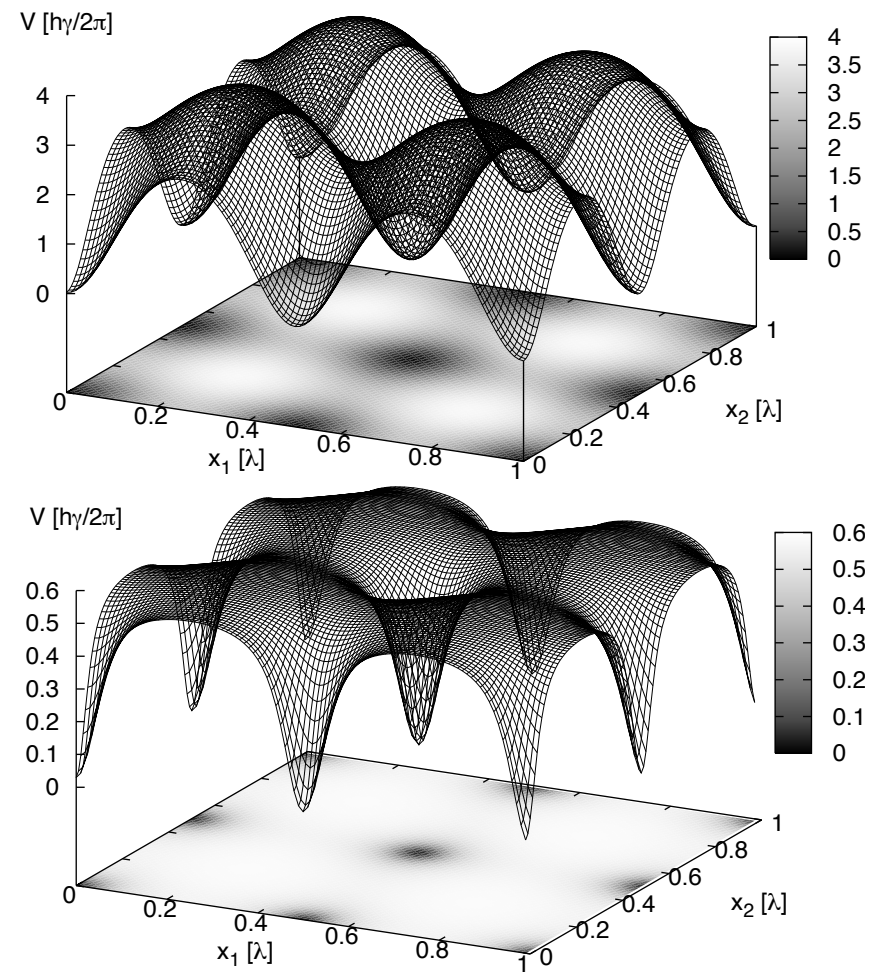

FIG. 7 The adiabatic cavity potential $V$ as a function of the atomic positions $x_{1}$ and $x_{2}$. The cavity is quasi-resonantly excited by a pump laser with detuning $\Delta_{C}=-\kappa+U_{0}$. The detuning from the atomic resonance is set to $\Delta_{A}=-50 \gamma$ to ensure the suppression of spontaneous photon scattering. In the upper graph, typical experimental cavity parameters $(\kappa=\gamma / 2, g=5 \gamma)$ (Münstermann et al., 2000) have been used, whereas in the lower graph $g$ was increased fourfold. In the first case, the potential is well approximated by a sum of two single-particle potentials. In the second case, either both atoms are trapped or free. From Asbóth et al. (2004).

et al., 2000). Although the single-atom light shift is comparable with the cavity linewidth $\left|U_{0}\right| \approx \kappa$, the effective interaction between the atoms is relatively weak and the potential resembles the familiar "egg-carton" surface proportional to $\sin ^{2}\left(k x_{1}\right)+\sin ^{2}\left(k x_{2}\right)$. For 'artificially' enlarged atom-field coupling $g=20 \gamma$, shown in Fig. 7 (lower graph), the atom-atom interaction strongly affects the potential landscape felt by the second atom, depending on the position of the first atom and vice versa. For this parameter setting the single-atom light shift is sufficiently large, $U_{0} \gg \kappa$, that removing one atom from the cavity antinode makes the potential experienced by the other atom vanish. Note that the trap is deeper for the smaller coupling of the upper graph. Interestingly, as was shown by Asbóth et al. (2004), the motion of the two atoms gets correlated even for the parameter setting of the upper graph as a consequence of additional nonconservative forces (see Sec. III.A.2). b. Atom pumping The situation drastically changes if the atoms are laser-driven from a direction perpendicular to the cavity axis. Intracavity photons are then created by Rayleigh scattering of laser photons into the cavity mode. Due to light interference, the scattered intracavity field exhibits a very sensitive dependence on the interatomic distance. For two atoms separated by odd integer multiples of the half-wavelength, the corresponding scattering amplitudes into the mode have the same magnitude but opposite sign, resulting in destructive interference and a vanishing cavity field amplitude. On the other hand, for atoms separated by even integer multiples of the half-wavelength, the field components scattered off the two atoms interfere constructively. Compared to the field intensity created by a single scatterer, the latter case yields a fourfold enhancement of the intensity, referred to as superradiance (DeVoe and Brewer, 1996; Dicke, 1954).

In the case of pumping directly the atoms the force along the cavity axis acting on the individual atoms due to light scattering cannot be expressed as a gradient of a collective potential, at variance to Eq. (20). One can admit this by checking that $\nabla_{i} \mathbf{F}_{j} \neq \nabla_{j} \mathbf{F}_{i}$, where $\nabla_{i}$ is the gradient with respect to the coordinate $\mathbf{r}_{i}$, and $\mathbf{F}_{j}$ is the force acting on atom $j$. If there was a potential $V$ such that $\mathbf{F}_{j}=-\nabla_{j} V$, the two sides should be equal as they are the second derivatives of the potential and the order of taking the derivatives is irrelevant according to Young's theorem. The fact that the force can not be derived from a potential is not so surprising, in hindsight, as we are dealing with an open system with continuous energy exchange with the environment and an unlimited energy resource in the form of the pump laser. Actually, the existence of a potential Eq. (20) for the cavity-driving geometry is the exceptional case.

Approximately, in the limit of $U_{0}, \Gamma_{0} \rightarrow 0$, more precisely $N^{2} U_{0} \ll\left(\kappa,\left|\Delta_{C}\right|\right)$, the motion of the atoms is governed by the collective potential

$$
V\left(\mathbf{r}_{1}, \ldots \mathbf{r}_{N}\right)=\hbar \frac{\eta_{\text {eff }}^{2} \Delta_{C}}{\Delta_{C}^{2}+\kappa^{2}}\left(\sum_{j=1}^{N} \cos \left(k x_{j}\right) \cos \left(k z_{j}\right)\right)^{2},
$$

where cosine mode functions were assumed for the cavity and the pump laser field. The interference effect is manifest: when scanning the atom-atom distance over a wavelength, the contrast of the interference in the cavity field intensity is unity regardless the atom-cavity coupling constant $g$. This is not the case for cavity pumping, where, in the limit of small coupling constant $g$, the atoms cause only a small modulation of the cavity intensity. Therefore, the atom pumping geometry lends itself to observe spectacular many-body effects even in the weak-coupling regime.

The superradiant light scattering into the cavity is the basis of various collective dynamical effects, which have been more profoundly studied theoretically. Although we 

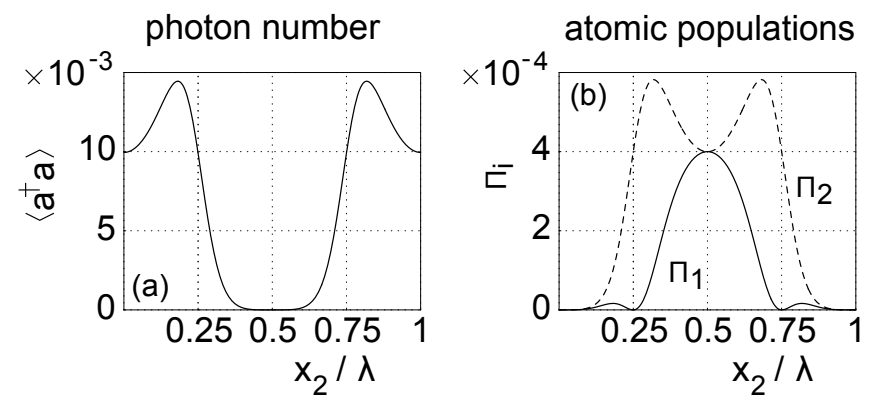

FIG. 8 Collective scattering of two atoms into the cavity mode including internal atomic excitation. (a) Mean number of photons scattered into the cavity mode at pump-cavity resonance, $\Delta_{C}=0$, with coupling strength $g \cos (k x)$. The atoms are placed at $x_{1}=0$ and $x_{2}$, respectively. (b) Excited state populations $\Pi_{1}$ and $\Pi_{2}$ of the two atoms (dashed and solid lines). The parameters are $\kappa=0.2 \gamma, \eta_{\mathrm{eff}}=\gamma, g=10 \gamma, \Delta_{A}=$ $100 \gamma$. From Zippilli et al. (2004b).

mostly neglect atomic saturation effects in this review, it is important to reveal modifications of the interference effect in the collective scattering when a small but finite atomic saturation is taken into account. Since the saturation also depends on the relative distance of the particles, new types of nonlinear behavior take place. For example, as shown in Fig. 8, the destructive interference for a separation of half-wavelength between the atoms is not perfect any more and the photon scattering generates a nonclassical cavity field with zero amplitude but finite photon number (Vidal et al., 2007; Zippilli et al., 2004a).

\section{Collective cooling, scaling laws}

As was discussed in Sec. II.B.1, the cavity cooling force on single atoms stems from a delicate correlation between the atomic motion and the retarded dynamics of the cavity field. In a many-atom system it is at first unclear what happens to these correlations in the presence of other moving atoms. Furthermore, the cavity-mediated crosstalk between atoms has a component sensitive to the atomic velocities (Domokos and Ritsch, 2003), i.e., atom 1 moving at velocity $v_{1}$ induces a linear friction force on atom 2, which might yield correlations in velocity space. To answer this question, one can straightforwardly generalize the semiclassical model, presented in Sec. II.A.3, for many atoms. In general, however, this leads to an analytically intractable problem. The dynamics of the many-atom system cannot be reduced to that of an effective mode, as was the case for the adiabatic potential Eq. (20) for atoms at rest. The two-atom case has been discussed in detail by Asbóth et al. (2004), who found, using the parameter regime of Fig. 7a, a buildup of strong correlations in the motion of two atoms due to the velocity-dependent cavity forces.

The scaling of the cavity cooling efficiency with the number of particles has been studied by means of numerical simulations for $N=1 \ldots 100$ in the limit of a weakly driven single-mode field, where the optical dipole potential negligibly perturbs the free motion of atoms along the cavity axis (Horak and Ritsch, 2001c). If the parameter $U_{0}$ is chosen sufficiently small so that the collective light shift is still below the cavity linewidth, $N U_{0}<\kappa$, the rate of kinetic energy dissipation is independent of the number of atoms. This suggests that the individual atoms in the cloud are cooled independently from each other, although they are all coupled to the same cavity mode. This holds only in the weak-coupling limit which is not practical for cooling since the cooling time is long.

When the collective coupling to the cavity mode is significant with respect to the linewidth $\kappa$, the scaling behavior of cooling with the number of atoms has been studied by keeping $N U_{0}$ and $\eta / \sqrt{N}$ constant while varying the atom number $N$. The former ensures an identical maximum collective light shift induced by the atoms, the latter amounts to a nearly constant optical potential depth (proportional to $U_{0} \eta^{2} / \kappa^{2}$ ). With this rescaling of the parameters, the effect of individual atoms on the cavity field diminishes as the number of atoms increases. The final temperature was found invariant, however, the cooling time increases linearly with the atom number $N$. As long as the driving $\eta$ is weak enough to result in a shallow optical potential depth, in which the atoms move almost freely, all the motional degrees of freedom along the cavity axis are cooled.

In the limit of tightly confined atoms, both theoretical calculations (Asbóth et al., 2004; Nagy et al., 2006a) and experiments (Schleier-Smith et al., 2011) proved that only the center-of-mass motion is damped by the cavityinduced friction force (Gangl and Ritsch, 1999, 2000). An efficient sideband cooling scheme has been proposed by Elsasser et al. (2003) for particles confined in the optical lattice potential generated by two counter-propagating degenerate modes of a ring cavity. The scheme relies on the collective atom-field coupling which lifts the degeneracy and creates two standing-wave modes phase-locked by the back-scattering of light. The lower-lying mode sustains the optical lattice with an intensity adjusted such that the upper-lying mode becomes resonant with the vibrational anti-Stokes Raman transition. The sideband cooling allows to reach the vibrational ground state.

A collective enhancement of friction on the center-ofmass motion has been demonstrated experimentally in the transverse pump configuration, as an accompanying effect of the self-organization into a Bragg-scattering lattice (see Sec. III.B.1). Peak decelerations of $-10^{3} \mathrm{~m} / \mathrm{s}^{2}$ have been observed, and the damping effect has been demonstrated with light-atom detunings up to $\Delta_{A} / 2 \pi=$ $-6 \mathrm{GHz}$. For smaller detunings $\Delta_{A} / 2 \pi \approx-160 \mathrm{MHz}$, similarly large velocity-dependent friction forces (up to decelerations of $-1500 \mathrm{~m} / \mathrm{s}^{2}$ and temperatures as low as $7 \mu \mathrm{K})$ have been observed in another set of experiments 


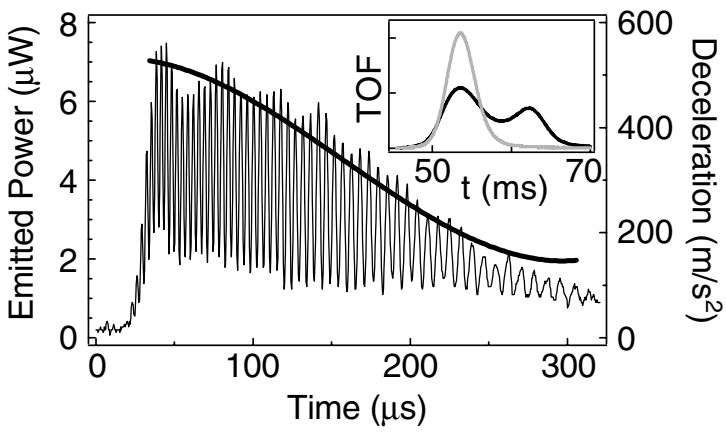

FIG. 9 Observation of collective friction force on the centerof-mass motion. The cavity output power (thin line, left scale) is shown during illumination of a freely falling atomic cloud (initial velocity $15 \mathrm{~cm} / \mathrm{s}$ ) with a transverse laser beam. The initial increase signifies the self-ordering into a Braggscattering lattice (see Sec. III.B.1). Deceleration of the centerof-mass motion is recorded via the beat signal recorded over $300 \mu \mathrm{s}$. The modulation stems from the spatial variation of the atom-cavity coupling with period $\lambda / 2$, whereby atoms cannot scatter at a node of the intracavity standing wave. The changing modulation frequency indicates the atomic deceleration (thick line, right scale). The inset shows the density profile (a.u.) of the atomic cloud after free expansion without (gray line) and with (black line) a $400 \mu$ s exposure to the pump beam. A fraction of about one third of the atoms is delayed significantly in accordance with the measured deceleration. Here the pump $I / I_{s}=420\left(I_{s}=1.1 \mathrm{~mW} / \mathrm{cm}^{2}\right.$ is the $\mathrm{D}$ line saturation intensity of $\mathrm{Cs}), \Delta_{A} / 2 \pi=-1.58 \mathrm{GHz}$, and $\Delta_{C} / 2 \pi=-10 \mathrm{MHz}$. The atom number is $N=2.6 \times 10^{7}$. From Black et al. (2003).

(Chan et al., 2003). Both the large friction and the low temperature cannot be explained in terms of the interaction between single atoms and the cavity field. In this detuning regime a theoretical description is more involved because the entire hyperfine manifold has to be taken into account and the interaction can lead to Raman lasing between different magnetic sublevels.

\section{Back-action, nonlinear dynamics}

In general, the interplay between the mechanical effect of light on the atomic motion and light scattering inside the cavity off the spatial atomic density distribution can lead to highly non-linear dynamics. Having a large atomic ensemble organized in a lattice structure, for example, the collective Bragg scattering much more efficiently redistributes the light between modes than Rayleigh scattering from the individual atoms. The enhancement factor, being on the order of the number of atoms, can give rise to significant sensitivity of light scattering to small variations of the spatial distribution. As an example, the back scattering between the counterpropagating modes (denoted by + and - ) of a ring cavity was found to depend strongly on the bunching parameter of the atomic distribution around the trapping sites of an optical lattice. In experiments performed by the Hamburg group (Elsasser et al., 2004; Nagorny et al., 2003), the amplitude $\alpha_{+}$in one of the modes was actively stabilized by a feedback loop, $\dot{\alpha}_{+}=0$, and thus the other mode obeyed the nonlinear equation of motion

$$
\dot{\alpha}_{-}=i N U_{0} B \frac{\alpha_{-}^{2}}{\alpha_{+}}-\kappa \alpha_{-}-i N U_{0} \alpha_{+} B^{*}+\eta_{-} .
$$

Here, $\eta_{-}$denotes the driving amplitude of this mode and $B=\left\langle e^{-2 i k z}\right\rangle$ the bunching parameter (see also Sec. III.B.2). For a thermal cloud the atomic bunching follows approximately $B \propto$ $\alpha_{-}^{*} /\left|\alpha_{-}\right| \exp \left\{-\right.$ const $\left./ \sqrt{\left|\alpha_{-}\right|}\right\}$. The resulting nonlinear dynamics was exemplified by a new kind of optical bistability in the dispersive atom-field coupling regime, which is outside the range of optical bistability effects relying on the nonlinearity of the internal atom-field coupling (Lugiato, 1984). Subsequent experiments (Klinner et al., 2006) also revealed the mechanical effect of light on the atomic distribution in the dispersive regime through the normal-mode splitting.

\section{B. Non-equilibrium phase transitions and collective instabilities}

The nonlinear collective dynamics of thermal atoms in a high-finesse resonator can give rise to non-equilibrium phase transitions and collective instabilities. In the following we present two, experimentally evidenced examples which have been theoretically studied both in the thermodynamic limit and by means of microscopic models.

\section{Spatial self-organization into a Bragg-crystal}

A thermal cloud of cold atoms interacting with a single mode of a high-finesse Fabry-Pérot cavity undergoes a phase transition upon tuning the power $P$ of a fardetuned laser beam (wavelength $\lambda$ ) which illuminates the atoms from a direction perpendicular to the cavity axis (Asbóth et al., 2005; Domokos and Ritsch, 2002). Below a threshold power $P_{\mathrm{cr}}$, the thermal fluctuations stabilize the homogeneous density distribution of the atomic cloud, and light which is scattered off the atoms into the cavity destructively interferes, rendering the mean cavity field amplitude to be zero. Above threshold, $P>P_{\mathrm{cr}}$, the atoms self-organize into a $\lambda$-periodic crystalline checkerboard order which is bound by the interference between the pump field and the macroscopic cavity field, resulting from Bragg scattering into the cavity mode.

This self-organization effect can be described in terms of a semiclassical model similar to Eq. (13), generalized to many atoms. A set of variables $\mathbf{p}_{j}$ and $\mathbf{r}_{j}$ is introduced, 
the index $j=1, \ldots, N$ labeling the atoms. For simplicity, the atomic motion is considered in two dimensions spanned by the cavity axis and the pump laser direction, with coordinates $x$ and $z$, respectively. The equation of motion for the coherent cavity field amplitude $\alpha$ is given by

$$
\begin{aligned}
\dot{\alpha}=i\left[\Delta_{C}-\right. & \left.U_{0} \sum_{j} \cos ^{2}\left(k x_{j}\right)\right] \alpha-\left[\kappa+\Gamma_{0} \sum_{j} \cos ^{2}\left(k x_{j}\right)\right] \alpha \\
& -i \eta_{\mathrm{eff}} \sum_{j} \cos \left(k x_{j}\right) \cos \left(k z_{j}\right)+\xi_{\alpha}, \quad \text { (23a) }
\end{aligned}
$$

where the effective pumping strength of the cavity mode is denoted by $\eta_{\text {eff }}=\frac{\Omega g \Delta_{A}}{\Delta_{A}^{2}+\gamma^{2}}$, see Eq. (10). Due to the interference term $\sum_{j} \cos \left(k x_{j}\right) \cos \left(k z_{j}\right)$ light scattering into the cavity vanishes for a homogeneous atomic density distribution. It can be small even if all the atoms are maximally coupled but the signs of the summands alternate. The light forces exerted on the individual atoms along the cavity and pump direction are given by

$$
\begin{gathered}
\dot{p}_{x_{j}}=-\hbar U_{0}|\alpha|^{2} \frac{\partial}{\partial x_{j}} \cos ^{2}\left(k x_{j}\right) \\
-\hbar \eta_{\mathrm{eff}}\left(\alpha+\alpha^{*}\right) \frac{\partial}{\partial x_{j}} \cos \left(k z_{j}\right) \cos \left(k x_{j}\right)+\xi_{x_{j}}, \\
\dot{p_{z j}}=-\hbar U_{0}(\Omega / g)^{2} \frac{\partial}{\partial z_{j}} \cos ^{2}\left(k z_{j}\right) \\
-\hbar \eta_{\mathrm{eff}}\left(\alpha+\alpha^{*}\right) \frac{\partial}{\partial z_{j}} \cos \left(k x_{j}\right) \cos \left(k z_{j}\right)+\xi_{z j},
\end{gathered}
$$

These equations include Langevin noise terms $\xi_{\alpha}, \xi_{x_{j}}$, and $\xi_{z j}$, defined by the non-vanishing second-order correlations,

$$
\begin{aligned}
\left\langle\xi_{\alpha}^{*} \xi_{\alpha}\right\rangle & =\kappa+\sum_{j=1}^{N} \Gamma_{0} \cos ^{2}\left(k x_{j}\right), \\
\left\langle\xi_{n} \xi_{\alpha}\right\rangle & =i \hbar \Gamma_{0} \partial_{n} \mathcal{E}\left(\mathbf{r}_{j}\right) \cos \left(k x_{j}\right), \\
\left\langle\xi_{n} \xi_{m}\right\rangle & =2 \hbar^{2} k^{2} \Gamma_{0}\left|\mathcal{E}\left(\mathbf{r}_{j}\right)\right|^{2} \overline{u_{n}^{2}} \delta_{n m}+\hbar^{2} \Gamma_{0} \\
& {\left[\partial_{n} \mathcal{E}^{*}\left(\mathbf{r}_{j}\right) \partial_{m} \mathcal{E}\left(\mathbf{r}_{j}\right)+\partial_{n} \mathcal{E}\left(\mathbf{r}_{j}\right) \partial_{m} \mathcal{E}^{*}\left(\mathbf{r}_{j}\right)\right], }
\end{aligned}
$$

with indices $n, m=x_{j}, z_{j}$. The noise terms associated with different atoms are not correlated. The complex dimensionless electric field is given by $\mathcal{E}(\mathbf{r})=\cos (k x) \alpha+$ $\cos (k z) \Omega / g$.

In Fig. 10 a numerical simulation of the trajectories of 40 atoms during the first $50 \mu \mathrm{s}$ of illumination are shown. The initial configuration is given by an ensemble of thermal atoms with random positions from a uniform, and velocities from a thermal distribution. The cavity mode initially is in the vacuum state $(\alpha=0)$. With the right choice of parameters the emergence of a periodic pattern in the spatial density distribution of the atoms

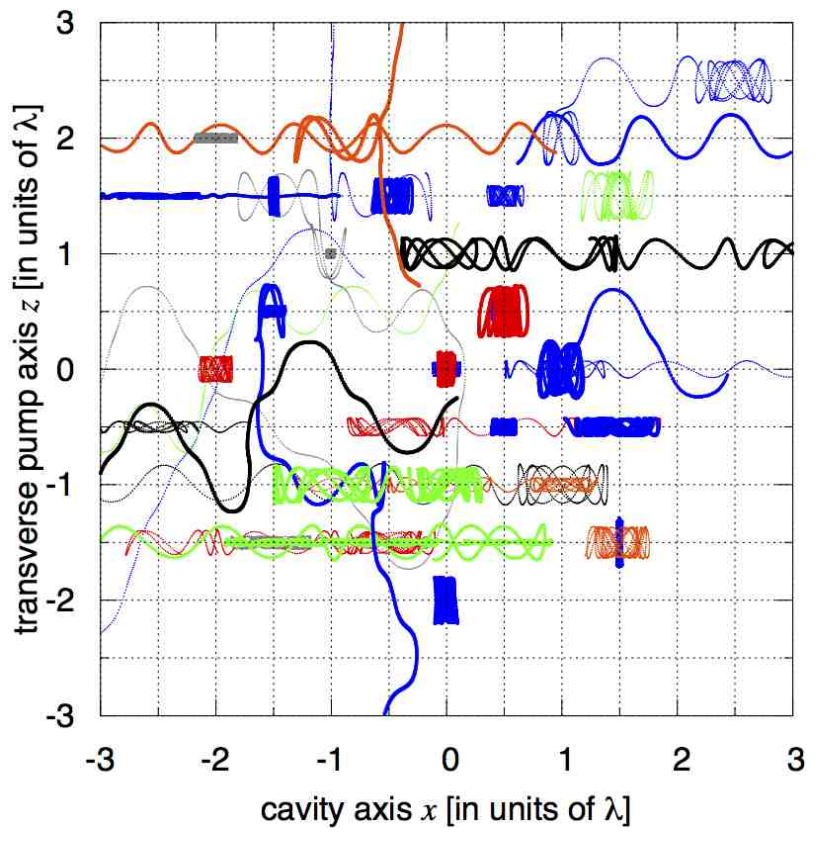

FIG. 10 (Color online) Self-organization of laser-driven atoms in a cavity. Numerically simulated two-dimensional trajectories during the first $50 \mu \mathrm{sec}$ of transverse illumination. A checkerboard pattern of trapped atoms emerges, in which the occupied trapping positions are separated by even multiples of $\lambda / 2$ (=1 edge). The grid lines indicate points of maximum coupling to the standing-wave cavity or pump field. There is a possible complementary configuration with atoms occupying the other set of intersections. Parameters: $\gamma=20 / \mu \mathrm{sec}$, $(g, \kappa)=(2.5,0.5) \gamma$, atomic detuning $\Delta_{A}=-500 \gamma$, cavity detuning $\Delta_{C}=-\kappa+N U_{0}$, and the pumping strength $\Omega=50 \gamma$. From Asbóth et al. (2005).

is observed, accompanied by the buildup of a coherent cavity field amplitude (Fig. 11). In the emerging configuration, assuming a red-detuned pump laser, the trapped atoms are oscillating about intensity maxima of the interfering pump-cavity field. Along the cavity and pump direction these are separated by even multiples of the optical wavelength. Since only the black or white fields of the underlying checkerboard lattice pattern are occupied, constructive interference leads to efficient Bragg scattering of pump photons into the cavity.

As will be shown in more detail later, the process of self-organization relies on the right choice of the detuning $\delta_{C}=\Delta_{C}-N U_{0} / 2$ between the pump laser and the dispersively shifted cavity resonance $\Delta_{C}$. For the case $\delta_{C}<0$, the potential term $\cos \left(k x_{j}\right) \cos \left(k z_{j}\right)$ in Eqs. (23b,c) attracts atoms towards the "majority" sites and repels them from the "minority" sites, providing positive feedback. Initiated by density fluctuations, one of the two possible Bragg lattices is then formed in a runaway process. For the case $\delta_{C}>0$, the scattered cavity field creates potential maxima (minima) at the positions of the majority (minority) sites, counteracting the amplification of density fluctuations and preventing a dynam- 

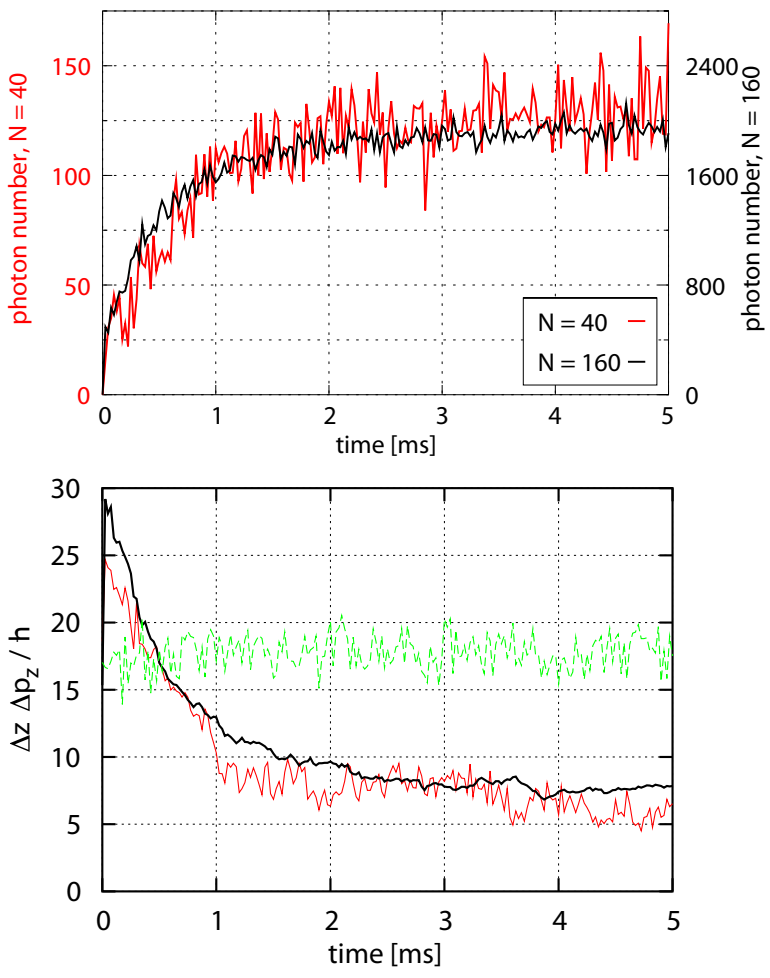

FIG. 11 (Color online) Cavity cooling in the self-organized phase. The time evolution of the photon number in the cavity (left) and the phase space density of the atoms (right) on a long time scale, for $N=40$ and $N=160$ atoms (different vertical scalings are used for illustrating the superradiance $\left.|\alpha|^{2} \propto N^{2}\right)$. In the right panel, the green curve fluctuating around a constant value corresponds to a uniformly distributed $N=40$ atoms driven below the self-organization threshold. The parameters are the same as in Fig. 10. From Asbóth et al. (2005).

ical instability. Furthermore, in this regime the delayed cavity response causes cavity heating of the atomic motion which obscures an equilibrium situation in the lack of other dissipative process.

For $\Delta_{C}-N U_{0}<0$, the initial fast buildup of a coherent cavity field continues over a longer timescale. The kinetic energy of the oscillating and the untrapped atoms dissipates owing to the cavity cooling mechanism, which leads to an increase of the number of trapped atoms and a stronger localization in the potential wells. This further improves coherent scattering into the cavity, as indicated by the slow increase in the cavity field intensity shown in Fig. 11. Comparing the time evolution of the intracavity photon number for the self-organization process of 40 and 160 atoms (rescaled in Fig. 11 by a factor of 16) demonstrates the superradiance effect, i.e. the field intensity scales cooperatively as the square of the particle number. The right panel of the figure shows that the cooling rate, described by the decrease of the phase space density of the atoms, is also similar for $N=40$ and $N=160$ and that the self-organization leads to smaller phase space densities than the homogeneous distribution below threshold.

Self-organization of laser-cooled atoms has been observed in experiments of the MIT group with $N \approx 10^{7}$ Cs atoms prepared at a temperature of $6 \mu \mathrm{K}$ in a nearly confocal Fabry-Pérot cavity (Black et al., 2003). Above a threshold intensity of the transverse pump beam, collective emission of light into the cavity was observed at a rate which exceeds the free-space single-atom Rayleigh scattering rate by a factor of up to $10^{3}$. This experiment clearly demonstrated the process of spontaneous symmetry breaking by measuring $\pi$-jumps in the phase of the emitted cavity field relative to the transverse pump field, corresponding to self-organization into the black or white lattice sites of a checkerboard pattern (Fig. 12). Retardation between the cavity field and the atomic motion resulted in a collective friction force on the center-of-mass degree of freedom. A deceleration of up to $1000 \mathrm{~m} / \mathrm{s}^{2}$ has been achieved with atom-cavity detunings as large as $\Delta_{A}=-2 \pi \times 1.58 \mathrm{GHz}$.

For finite atom number $N$ and finite measurement time, an interesting hysteresis effect accompanies selforganization, as shown in Fig. 13. The thermodynamic limit $N \rightarrow \infty$ is approached by simulations of Eq. (23) with the atomic density $N / V \propto N g^{2}$ and the cavity loss rate kept constant. The percentage of defect atoms after $4 \mathrm{~ms}$ of simulation time as a function of the pumping laser strength clearly shows the transition. However, the transition point is dependent on $N$ and on whether

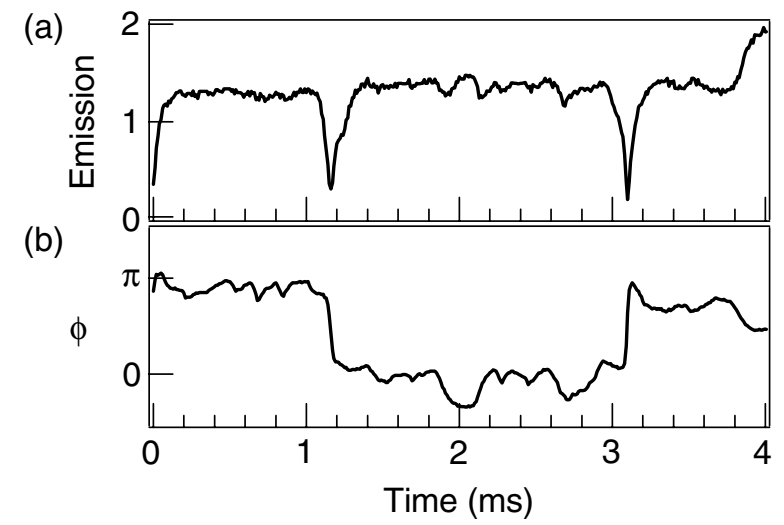

FIG. 12 Observation of spontaneous symmetry breaking in the self-organization phase transition. Simultaneous time traces of the (a) intracavity intensity (in arbitrary units) and (b) the relative pump-cavity phase. Drops in the intensity correspond to time intervals during which the beams of the magneto-optical trap (MOT) are switched on forcing the atomic density distribution to randomize. After switching off the MOT beams, the atoms self-organize again into one of the two possible checkerboard patterns, as indicated by the relative phase signal. Experimental parameters were $N=8.2 \times 10^{6}, \Delta_{A}=-2 \pi \times 1.59 \mathrm{GHz}, \Delta_{C}=-2 \pi \times 20 \mathrm{MHz}$, and $I / I_{\text {sat }}=440$. From Black et al. (2003). 


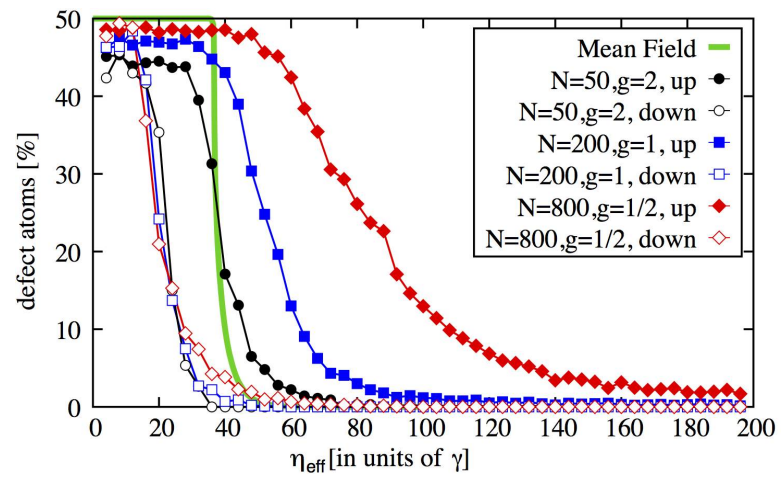

FIG. 13 (Color online) Hysteresis effect for finite measurement time. Ratio of atoms in the "defect" positions against pumping strength $\eta$, $4 \mathrm{~ms}$ after the loading of the trap with a uniform ("up") or organized ("down") gas of atoms. The different curves show the approach towards the thermodynamic limit. The parameters are $\kappa=\gamma / 2, \Delta_{A}=-500 \gamma$, $N g^{2}=200 \gamma^{2} \Delta_{C}=-\kappa-N g^{2} /\left|\Delta_{A}\right|, k_{B} T=\hbar \kappa$. From Asbóth et al. (2005).

the initial positions were uniformly distributed ("up") or at "odd" points of maximal coupling ("down"). The breadth of the hysteresis increases with the atom number, but decreases with the measurement time. This behavior can be explained by taking into account statistical fluctuations arising from the finite atom number $N$. Assuming that the self-organization from a uniform distribution ("up" curves) is triggered when the fluctuating energy difference between the even and odd sites momentarily exceeds the mean kinetic energy, a scaling $\mathrm{Ng}^{4}$ of the threshold with the pump intensity was found in accordance with the numerical results of the plot. The disappearance of the lattice pattern for decreasing pump power ("down" curves) when the system is started from the ordered phase occurs at the half of the mean-field threshold (see Eq. (33) in the next subsection), independently of the atom number $N$.

Self-organization of laser-driven atoms occurs also in a transversally driven ring-resonator geometry supporting two running-wave modes (Nagy et al., 2006b). In contrast to the linear single-mode cavity case, here the transition from the homogeneous to the organized density distribution involves spontaneous breaking of a continuous (rather than a discrete) translational symmetry (see also IV.D.3).

\section{Collective atomic recoil laser}

Collective atomic recoil lasing (CARL) is the prominent many-body instability effect in a ring cavity, originally predicted by Bonifacio et al. (1994). An ensemble of cold atoms couples to two counter-propagating modes of a unidirectionally pumped high-finesse ring cavity. Light scattering off the atomic ensemble between these cavity modes leads to a collective instability corresponding to an exponential gain for the back-propagating field mode amplitude in conjunction with an atomic bunching at the antinodes of a self-organized optical lattice. In the presence of dissipation of the atomic kinetic energy, a steady-state operation of CARL can be achieved with a self-determined atomic drift velocity and back-reflected light frequency.

The CARL scheme involves an interplay between the influence of the atomic motion on the Rayleigh scattering of light and, reversely, the mechanical effect of light upon the atomic motion. The former effect has been previously seen, for example, as the so-called recoil induced resonance (RIR) in the transmission spectrum of a probe beam making a small angle with a one-dimensional lin $\perp$ lin optical molasses (Courtois et al., 1994), and also in a optical dipole trap formed by counter-propagating modes of a ring cavity (Kruse et al., 2003b). This narrow, dispersion-like resonance around the pump field frequency originates from a two-photon Raman transition between different momentum states of the atoms. Different populations of the corresponding momentum states lead to gain or attenuation of a probe beam. For a thermal velocity distribution, a probe frequency tuned slightly below the pump frequency gives rise to gain, whereas for negative detuning there is an attenuation of the probe. The probe transmission spectrum measurement provides information about the temperature, and even more about the velocity distribution (Brzozowska et al., 2006).

It has been predicted that, based on the RIR gain effect, a weak probe field injected in the direction opposite to the strong pump field would exponentially amplify due to the self-bunching of a part of the atoms into a lattice which Bragg reflects the strong pump beam more efficiently than single atoms (Bonifacio et al., 1994). However, one needs long enough interaction time so that back-action of the light scattering on the velocity distribution can have a significant effect (Berman, 1999). This can be accomplished, for example, by confining the light modes into a cavity. Lasing mediated by the collective atomic recoil between the counter-propagating modes of a unidirectionally ring cavity has been observed (Kruse et al., 2003a). Back-action on the atomic motion has been demonstrated by detecting the displacement of the atoms accelerated by the momentum transfer process. The self-consistent solution is an accelerating Bragg-lattice of atoms co-moving with the standing wave formed by the pump and the back-reflected component having a Doppler-shifted frequency $\Delta \omega=2 k v$ with respect to the pump. The phase dynamics of the counter-propagating modes can be monitored as a beat signal between the outcoupled beams, which reveals the acceleration by an increasingly red-detuned probe as a function of time (Fig. 14).

The runaway process can be counteracted by introduc- 

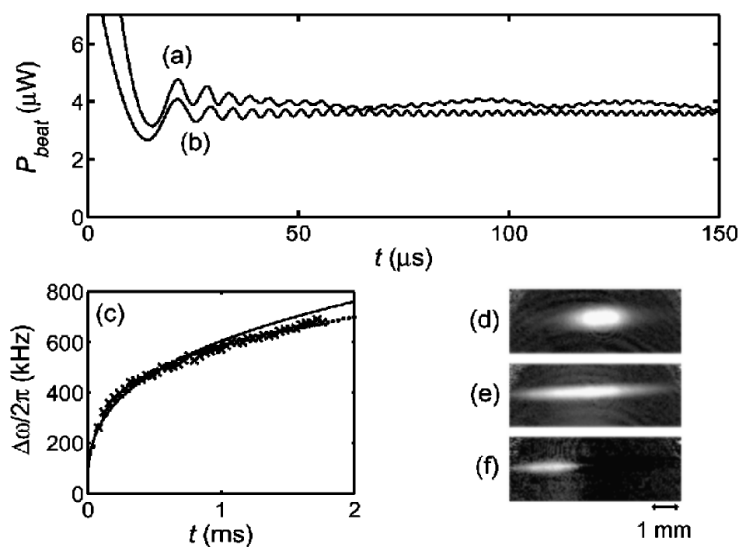

FIG. 14 Observation of lasing mediated by collective atomic recoil. (a) Recorded time evolution of the observed beat signal between the $\alpha_{+}$and $\alpha_{-}$cavity modes of a ring cavity. Initially both modes are pumped to form an optical lattice of $N=10^{6}$ atoms. The initial drop is due to the decay of the unpumped mode after switching off the $\alpha_{-}$pump at $t=0$. Well beyond the ring-down time of about $10 \mu \mathrm{s}$, the persisting oscillations demonstrate the coherent backscattering of the pumped mode. (b) Numerical simulation with the temperature adjusted to $200 \mu \mathrm{K}$. (c) The symbols $(\times)$ trace the evolution of the beat frequency after switch-off (dotted line is from numerical simulation). The increase of the beat frequency corresponds to the acceleration of the Bragg lattice of back reflecting atoms, which lasts until the Doppler-shifted frequency drops out of cavity resonance. Absorption images of a cloud of $6 \times 10^{6}$ atoms recorded at (d) $0 \mathrm{~ms}$ and (e) $6 \mathrm{~ms}$ after switching off the probe beam pumping. All images are taken after a $1 \mathrm{~ms}$ free expansion time. (f) This image is obtained by subtracting from image (e) an absorption image taken $6 \mathrm{~ms}$ after switch-off with low cavity finesse for which no collective recoil is expected. The intracavity power has been adjusted to the same value as in the high finesse case. From Kruse et al. (2003a).

ing some external friction force on the atom motion. The dissipation gives rise to a steady-state solution which involves a constant drift of the entire atomic cloud at a speed $v$. Accordingly, we transform the atomic position variables such as $z_{j}=\tilde{z}_{j}+v t$, and the coherent field amplitude of the running-wave field mode propagating opposite to the pumped field mode as $\alpha_{-}=\tilde{\alpha}_{-} e^{2 i k v t}$. The drift velocity $v$ is to be determined in a self-consistent manner. The semiclassical equations describing atomic motion are given by

$$
\dot{p}_{j}=-\frac{\beta}{m} p_{j}+\hbar U_{0} 2 i k\left(\alpha_{+}^{*} \tilde{\alpha}_{-} e^{-2 i k \tilde{z}_{j}}-\tilde{\alpha}_{-}^{*} \alpha_{+} e^{2 i k \tilde{z}_{j}},\right)
$$

where $\beta$ represents the linear friction arising, e.g., from collisions with a buffer gas (Bonifacio, 1996; Perrin et al., 2001) or laser-cooling in an optical molasses (Kruse et al., 2003a). The cavity field amplitudes evolve as

$$
\begin{aligned}
& \dot{\alpha}_{+}=\left(i \delta_{C}-\kappa\right) \alpha_{+}-i N U_{0} B \tilde{\alpha}_{-}+\eta, \\
& \dot{\tilde{\alpha}}_{-}=\left[i\left(\delta_{C}-2 k v\right)-\kappa\right] \tilde{\alpha}_{-}-i N U_{0} B^{*} \alpha_{+},
\end{aligned}
$$

where $\delta_{C}=\Delta_{C}-N U_{0}$ is the effective detuning of the pump frequency from the atoms-shifted cavity resonance. The atomic positions enter through the bunching parameter

$$
B=\frac{1}{N} \sum_{j=1}^{N} e^{-2 i k \tilde{z}_{j}} \equiv b e^{-i \varphi} .
$$

A closed set of equations can be formed in which the atomic cloud is characterized by the three real parameters $v, b$, and $\phi$. A trivial solution of these equations corresponds to the case where the atoms are uniformly distributed in space $(b=0, v=0)$ and the counterpropagating field mode amplitude vanishes $\left(\alpha_{-}=0\right)$. A non-trivial steady-state solution can be obtained numerically from the coupled algebraic equations (time derivatives set to zero). This solution can be approximated analytically by assuming perfect bunching, $b=1$ and $\varphi=0$. The only remaining free parameter is then given by the steady-state drift velocity $v$ which obeys the algebraic equation

$$
2 k v=8 \frac{m \omega_{R}}{\beta} N U_{0}^{2} \frac{\kappa|\eta|^{2}}{|D|^{2}},
$$

where $D=\left(i \delta_{C}-\kappa\right)\left[i\left(\delta_{C}-2 k v\right)-\kappa\right]+N^{2} U_{0}^{2} b^{2}$. In order to gain insight into the solution, the following simplifications can be made: (i) neglect in $D$ the last $U_{0}^{2}$ term originating from (second-order) scattering of the $\alpha_{-}$mode back into the pumped $\alpha_{+}$one, and (ii) consider resonance $\delta_{C}=0$. Then one can identify the typical solutions of two different regimes. In the limit of a large Doppler shift, $k v \gg \kappa$, the drift velocity and the back reflected power scale with the atom number as $v \propto N^{1 / 3}$ and $\left|\alpha_{-}\right|^{2} \propto N^{4 / 3}$, respectively. This is referred to as the CARL limit in the literature. In the opposite limit of a small Doppler shift, $k v \ll \kappa$, the velocity obeys $v \propto N$ and the intensity exhibits superradiant behavior, $\left|\alpha_{-}\right|^{2} \propto N^{2}$, corresponding in this geometry to Bragg retro-reflection. The relation between these two kinds of superradiant instabilities in the collective interaction of light with an atomic gas has been established experimentally by Slama et al. (2007a,b). While superradiant Rayleigh scattering from atomic clouds is normally observed only at very low temperatures, i.e., well below $1 \mu \mathrm{K}$, (Inouye et al., 1999), the presence of the ring cavity enhances cooperativity and allows for superradiance with thermal clouds as hot as several $10 \mu \mathrm{K}$.

In the experiments of the Tübingen group (Cube et al., 2004; Kruse et al., 2003a), an optical molasses has been used to impose a motional damping force on the atoms. In such a viscous CARL system, there is a steady-state 

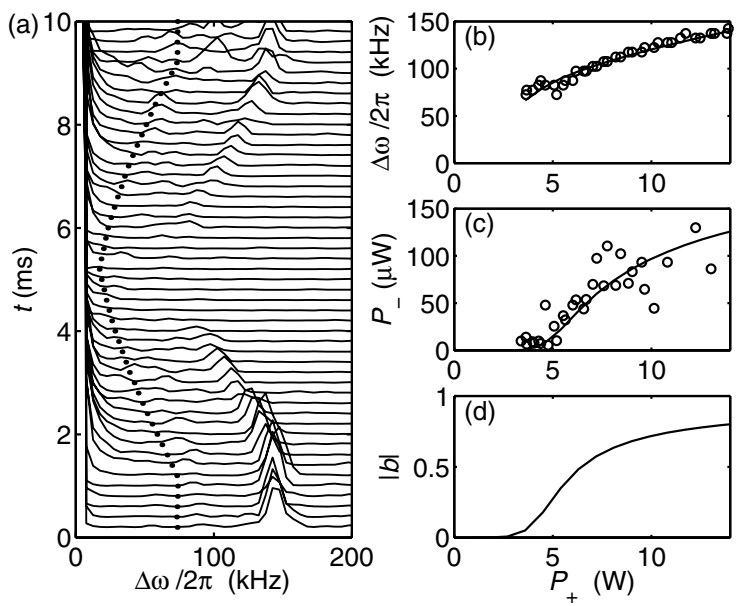

FIG. 15 Pump power threshold for the collective atomic recoil lasing. (a) Sectionwise Fourier-transform of the interference signal $P_{\text {beat }}$ with the pump laser power being ramped down and up (the dotted line is proportional to the pump laser power). At $t=0$ the system is in the ordered CARL phase, by gradually decreasing the power the drift velocity decreases (the peaks in the Fourier spectra shift downward) until the back reflection ceases at a threshold pump power. Ramping up the pump power from about $t=5 \mathrm{~ms}$, the appearance of a peak is delayed and occurs at about the same threshold value of the pump power. (b) Dependence of the CARL frequency on the intracavity pump power. (c) Dependence of the probe field intensity on the pump power. The CARL laser threshold is around $P_{+}=4 \mathrm{~W}$ intracavity power. The fitted curves are based on a Fokker-Planck theory outlined in Sec. III.C.1. (d) Calculated bunching parameter. The parameters are $\beta=4 \kappa$, $N=10^{6}$ atoms, $\Delta_{A}=-2 \pi \times 1.7 \mathrm{THz}$ and $T=200 \mu \mathrm{K}$. From Cube et al. (2004).

operation with a self-consistent drift velocity, according to Eq. (26), at which the friction compensates the acceleration due to back scattering of photons. The Fourier spectrum of the beat signal between the pumped mode and the counter-propagating one in steady-state is shown in Fig. 15a for various pump strengths. The drift velocity can be deduced from the Doppler-shift of the backreflected field as shown in Fig. 15b, the corresponding range of velocities is from 7 to $13 \mathrm{~cm} / \mathrm{s}$. The beat frequency as well as the drift frequency vary as a function of the pump power. However, the dramatic feature of Fig. 15 is the clear appearance of a threshold: the selfbunching and backscattering starts only above a welldefined threshold pump power. This measurement provides thus an experimental evidence of a phase transition to a state of synchronized atomic motion (Cube et al., 2004). Underlying the critical behavior is the diffusion accompanying friction: the spontaneous photon scattering of the molasses laser beams leads to a random heating force which stabilizes the homogeneous distribution and thereby can prevent the formation of a Bragg-lattice for weak pump power (Robb et al., 2004). Above thresh- old, the dissipation and the fluctuations together lead to a position distribution which exhibits a finite bunching parameter. These effects are discussed in the framework of various mean-field theories for the atomic position distribution (see Sect. III.C).

We note that the CARL system and its phase transition has been studied also in the case where the atomic transition frequency is close-to-resonance with the pump laser field (Perrin et al., 2001). Then the atomic polarization plays a dynamical role and the transition does not require spatial bunching but the emergence of coherent polarization grating (Perrin et al., 2002).

The viscous CARL transition exhibits analogy with that of the generic Kuramoto model which describes the self-synchronization of coupled oscillators with different frequencies (Kuramoto, 1975; Strogatz, 2000). To reveal the analogy, one can transform the CARL equations using the following assumptions: (i) the motion is overdamped $\left(\dot{p}_{j}=0\right)$; (ii) the pumped field amplitude $\alpha_{+}$is a constant of time $\left(\alpha_{+} \approx \eta / \kappa\right)$; (iii) the counterpropagating mode amplitude is stationary, oscillating at frequency $\omega_{0}$, i.e. , effectively $\dot{\tilde{\alpha}}_{-}=-i \omega_{0} \tilde{\alpha}_{-}$with the frequency $\omega_{0}$ being determined by the constant drift velocity; and (iv) $\kappa \ll \omega_{0}$. With these assumptions and by using the notation $\theta_{j}=2 k \tilde{z}_{j}$, Eqs. (25) simplify to

$$
\dot{\theta}_{j}=\frac{2 k}{m \beta} \xi+K b \sin \left(\varphi-\theta_{j}\right),
$$

which is formally equivalent with the Kuramoto model. The Langevin-type random noise $\xi$, associated with the friction term $-\beta p$ in Eq. (25a), introduces the random frequencies present in the Kuramoto-type systems. The coupling strength is $K=2 \omega_{R} N U_{0}^{2}\left|\alpha_{+}\right|^{2} /\left(\omega_{0} \beta\right)$. The mean-field character is obvious: each oscillator couples only to the mean-field quantities $b$ and $\varphi$. The phase $\theta_{j}$ is pulled toward the mean-field phase $\varphi$, which increases the order parameter $b$. The coupling is proportional to $b$, which sets a positive feedback loop. With the increasing coherence $b$, even more oscillators can be recruited to the synchronized pack (those being within the bandwidth $K b$ ), further increasing $b$. Such a runaway process starts only above a critical coupling $K$.

\section{Phase-space and mean-field descriptions for large particle numbers}

Due to the nonlinearity of the coupled atom-field dynamics, exact analytic results for atomic ensembles coupled to optical cavities are rather sparse and the computational demand often hinders simulations at realistic particle numbers (Deachapunya et al., 2008; Salzburger and Ritsch, 2009). In the following, we will present meanfield methods which allow to predict instability thresholds and to model the effective dynamics in the thermodynamic limit. Starting with the assumption of a quasi- 
thermal distribution, the critical behavior can be revealed and the threshold for criticality can be approximated. We will continue by adopting phase-space methods that can account for general position and velocity distributions. A Vlasov-type equation allows for a more accurate estimation of the threshold, and also for performing stability analysis and setting up a phase diagram. This analysis leads to such intriguing predictions that the cavitymediated interaction combined with the cavity cooling effect can be the basis of a generally applicable sympathetic cooling scheme. Finally, a Fokker-Planck-type equation can be constructed in order to determine the steady-state of the non-equilibrium systems in the thermodynamic limit. The methods show similarities with plasma physics where equally complex, coupled dynamics of particles and fields occurs (Montgomery, 1971).

\section{Critical point}

The simplest mean-field model is based on the assumption that the atomic motion is overdamped and the distribution function of the atomic positions $\rho(x, t)$ is a thermal distribution. The critical point of the CARL instability (Sec. III.B.2) and that of the self-organization (Sec. III.B.1) can be calculated with this approach.

a. Dynamical equations The motional damping is characterized by a linear friction coefficient $\beta$ (half of the kinetic energy damping rate is $\beta / m$ with $m$ being the atomic mass) and a temperature $T$. Then the mean atom density distribution obeys the Smoluchowski-equation

$$
\frac{\partial \rho(x, t)}{\partial t}=-\frac{1}{\beta} \frac{\partial}{\partial x}\left[F(x) \rho(x, t)-k_{B} T \frac{\partial \rho(x, t)}{\partial x}\right] .
$$

In the ring cavity geometry of CARL (Kruse et al., 2003a), for example, the force $F(x)$ is given by the last term on the right-hand side of Eq. (25a). It contains the field mode amplitudes which couple back to the atomic density distribution via the bunching parameter $\mathcal{B}$,

$$
\begin{aligned}
\dot{\tilde{\alpha}}_{-} & =-\kappa \tilde{\alpha}_{-}-i U_{0} \alpha_{+} \mathcal{B} \\
\text { with } \quad \mathcal{B} & =\int_{0}^{\lambda} d x \rho(x, t) e^{2 i k x} .
\end{aligned}
$$

For simplicity, the center-of-mass velocity and the detuning $\delta_{C}$ were set to zero. Linear perturbation calculus leads to the instability threshold of the homogeneous solution (Robb et al., 2004). Since the spatial coupling functions are sinusoidal, only a few Fourier components are involved in the initial dynamics. In particular, in order to determine the instability of the homogeneous distribution, only the single mode function $e^{-i k x}$ of the counter-propagating cavity mode needs to be taken into account. In the so-called CARL limit (see Sec. III.B.2), one obtains the following threshold condition for the cavity pump amplitude:

$$
\eta^{2} \geq\left(\frac{k_{B} T}{\hbar}\right)^{3 / 2} \sqrt{\frac{m \omega_{R}}{\beta}} \frac{\kappa^{5 / 2}}{N U_{0}^{2}} .
$$

b. Canonical distribution A further simplification can be made in the mean-field approach if $\kappa$ is the largest rate in the dynamics. Adiabatic elimination of the cavity field dynamics then results in a self-consistent optical potential $V(x)$ in which the spatial density $\rho(x)$ of the atoms is determined by a canonical distribution,

$$
\rho(x)=\frac{1}{Z} \exp \left(-V(x) /\left(k_{B} T\right)\right),
$$

with the partition function $Z=\int \exp \left(-V(x) /\left(k_{B} T\right)\right) d x$ ensuring normalization of $\rho(x)$ to unity. The temperature could be identified with the one which is achieved in cavity cooling, $k_{B} T \approx \hbar \kappa$, but in general it can be set by other means, e.g., by laser-cooling in an external optical molasses. The nonlinearity enters the equations through the dependence of the potential $V(x)$ on the atomic density $\rho(x)$ itself, i.e. , $V=V(x, \rho(x))$.

In principle, the optical force acting on the atoms in the cavity does not derive from a potential when the back-action of the atomic motion on the radiation field amplitude is significant. As was noted by Asbóth and Domokos (2007), dynamical equations based on forces, such as Eq. (28) and Eq. (29a) have to be used. However, in the spirit of the mean-field approach, the effect of an individual atom on the field amplitude is negligible with respect to the summed effect of all the others. In the limit of many atoms with small single-atom coupling, the motion of a single atom is very well described by an effective potential determined by the many-body ensemble.

For the example of self-organization in a standing-wave cavity, see Sec. III.B.1, the light potential along the cavity axis is given by

$$
V(x)=U_{2} \cos ^{2}(k x)+U_{1} \cos (k x),
$$

which is composed of the sum of a $\lambda / 2$-periodic potential stemming from the cavity field and a $\lambda$-periodic one arising from the interference between cavity and pump fields. The depths of these potentials are

$$
\begin{aligned}
& U_{2}=N^{2}\langle\cos (k x)\rangle^{2} \hbar I_{0} U_{0} \\
& U_{1}=2 N\langle\cos (k x)\rangle \hbar I_{0}\left(\Delta_{C}-N U_{0}\left\langle\cos ^{2}(k x)\right\rangle\right),
\end{aligned}
$$

where $I_{0}$ is a dimensionless single-atom scattering parameter, $I_{0} \propto \eta^{2}$. Equation (30) has to be solved in a self-consistent manner by iteration. In Asbóth et al. (2005), the threshold has been analytically determined to be

$$
\eta_{\mathrm{eff}, \mathrm{c}}^{2}=\frac{k_{B} T}{\hbar} \frac{\left(\kappa^{2}+\delta_{C}^{2}\right)}{N\left|\delta_{C}\right|}
$$




\section{Stability analysis and phase diagram}

In the following we will account explicitly for the effect of the velocity distribution on the dynamics and on the instability threshold. A mean-field model based on the Vlasov-equation for the phase space distribution $f(x, v, t)$ has been derived from a microscopic theory for the infinite system size by Grießer et al. (2010). For onedimensional motion along the cavity axis, the dynamical equation reads

$$
\frac{\partial f}{\partial t}+v \frac{\partial f}{\partial x}-\partial_{x} \phi(x, \alpha) \frac{\partial f}{\partial v}=0
$$

where $\phi(x, \alpha)$ is the potential corresponding to a momentary field amplitude $\alpha$. For the generic example of a laser-driven cold atomic cloud in a single-mode standingwave resonator with mode function $\cos (k x)$, the potential is

$$
\phi(x, \alpha)=\frac{2 \hbar}{m}\left(\frac{U_{0}}{4}|\alpha|^{2} \cos (2 k x)+\eta_{\mathrm{eff}} \operatorname{Re}(\alpha) \cos (k x)\right) .
$$

Similarly to the adiabatic potential, Eq. (31), there is a $\lambda / 2$ and a $\lambda$-periodic term, this latter originates from interference between the transverse pump laser and the intracavity field. However, in this more general approach the cavity field amplitude is kept dynamical obeying the self-consistent equation

$$
\begin{aligned}
\dot{\alpha}=( & \left.\kappa+i \delta_{C}\right) \alpha+\eta \\
- & i \alpha \frac{N U_{0}}{2} \int_{-\infty}^{\infty} d v \int_{0}^{\lambda} \cos (2 k x) f(x, v, t) d x \\
& -i N \eta_{\mathrm{eff}} \int_{-\infty}^{\infty} d v \int_{0}^{\lambda} \cos (k x) f(x, v, t) d x
\end{aligned}
$$

where $\delta_{C}=\Delta_{C}-N U_{0} / 2$. This approach based on the Vlasov-equation is well suited to study the mean-field dynamics at short times in order to test the stability of stationary states. Over longer time scales, statistical fluctuations have to be taken into account in the framework of a Fokker-Planck equation for the velocity distribution, which is presented in Sec. III.C.3.

a. Nonlinear response of a cold atomic cloud in a driven Fabry-Pérot cavity For cavity pumping only $\left(\eta_{\text {eff }}=\right.$ $0, \eta \neq 0)$, the self-consistent steady-state solution exhibits a strong nonlinear optical response. Underlying the nonlinearity, the particle distribution and thus the effective refractive index of the cloud depends on the cavity pump intensity. Above a sufficient pump strength, multiple stationary solutions appear, reminiscent of optical bistability. One can perform a systematic stability analysis of these solutions by studying the dynamics of small fluctuations of the field and the particle distribution (Grießer and Ritsch, 2011). As shown in Fig. 16, the
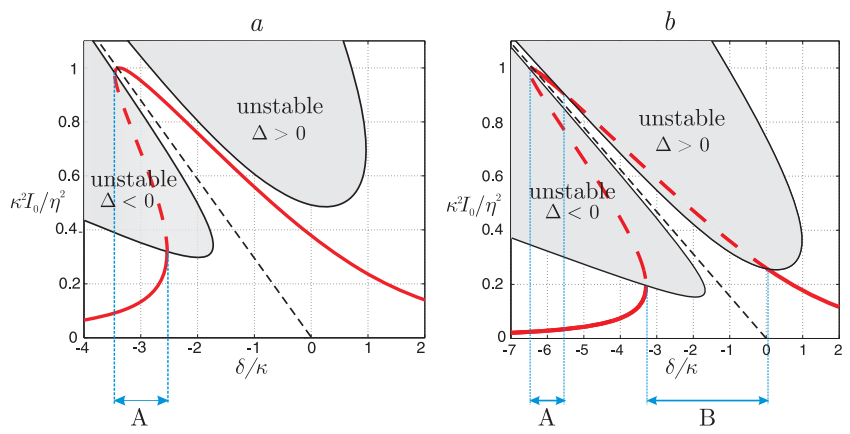

FIG. 16 (Color online) Normalized solutions for the steadystate photon number (red) $I_{0}=|\alpha|^{2}$ versus effective cavity detuning $\delta=\delta_{C}-N U_{0}$ for a thermal gas in a driven standingwave cavity. The driving strength is $\eta=13 \kappa$ (a) and $\eta=18 \kappa$ (b). Those parts of the response curve that lie inside the instability region (shaded area) are depicted in dashed and correspond to linearly unstable steady states. The intervals designated A correspond to bistability, the interval designated B supports no stable steady state at all. The parameters are $N=10^{5}, U_{0}=0.04 \kappa, \eta=18 \kappa, \kappa=2000 \omega_{R}$ and $k_{B} T=\hbar \kappa$. From Grießer and Ritsch (2011).

stability analysis reveals regions of bistability as well as parameter ranges where no stable solutions exist.

In the parameter regions of instability, the numerical solution of the dynamical Vlasov equation reveals a limit cycle behavior with subsequent appearance of higher frequencies than the fundamental cycle (Grießer and Ritsch, 2011). In the quantum regime essentially the same behavior is retrieved with the recoil frequency determining the oscillation frequencies $\nu \approx 4 \omega_{R}$ (Ritter et al., 2009) (see Sec. IV.C).

b. Self-organization of a laser-driven cloud of atoms For a purely transverse pump geometry $\left(\eta=0, \eta_{\text {eff }} \neq 0\right)$, one can systematically recalculate the critical pump amplitude $\eta_{\text {eff,c }}$ that marks the transition from the stable regime to the unstable one, where small fluctuations are amplified and grow exponentially.

The Vlasov equation Eq. (34) together with the equation for the coherent cavity field amplitude $\alpha$ possesses an infinite number of stationary solutions with a spatially homogeneous density distribution and zero cavity field but different velocity distribution, which, however, are not necessarily stable against fluctuations. Indeed, any symmetric velocity distribution $g\left(v / v_{\mathrm{T}}\right)=L v_{\mathrm{T}} f(v)$ for $\delta_{C}<0$ is stable only if

$$
\frac{N\left|\eta_{\mathrm{eff}}\right|^{2}}{k_{\mathrm{B}} T} \operatorname{vp} \int_{-\infty}^{\infty} \frac{g^{\prime}(\xi)}{-2 \xi} d \xi<\frac{\delta_{C}^{2}+\kappa^{2}}{\hbar\left|\delta_{C}\right|}
$$

where vp denotes the Cauchy principal value. Here we have defined the thermal velocity $v_{\mathrm{T}}^{2}=2 k_{\mathrm{B}} T / m ; L$ denotes the cavity length. For a Gaussian distribution the integral evaluates to one, and the condition is equivalent to Eq. (33). 

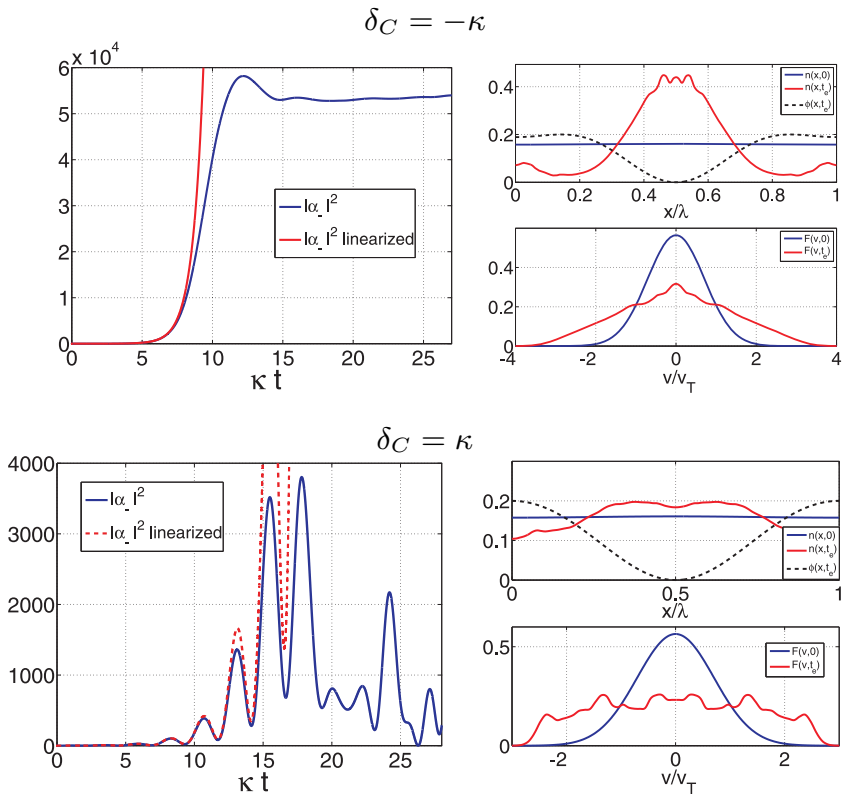

FIG. 17 (Color online) Laser illuminated cold gas in a ringcavity: time evolution of the intra-cavity field intensity (left), and the instantaneous spatial $n(x)$ (upper right) and velocity $F(v)$ (lower right) distributions of the particles along the cavity axis at times $t=0$ and $t_{e}=28 / \kappa$ for $\delta_{C}=-\kappa$ (upper row) and $\delta_{C}=\kappa$ (bottom row). For $\delta_{C}<0$, after a transient exponential growth, the field intensity saturates accompanied by the trapping of atoms in the self-organized pattern. By contrast, for $\delta_{C}>0$ the transient exponential growth is followed by oscillations. Parameters are $N=10^{4}, U_{0}=-\kappa / N$, $\eta_{\text {eff }}=0.05 \kappa, k v_{T}=\kappa$, and $v_{R}=v_{T} / 5$. From Grießer et al. (2010).

Figure 17 shows the results of a numerical simulation of Eqs. (34), (35) and (36), initialized with a perturbed gaussian distribution

$$
f(x, v, 0)=\frac{1}{\lambda \sqrt{\pi} v_{T}} e^{-v^{2} / v_{T}^{2}}(1-\epsilon \cos (k x)),
$$

with $\epsilon \ll 1$, for the case of a transversely pumped ring cavity, where the light can be scattered into a superposition of two resonant cavity modes. Apart from possessing continuous translational symmetry, the dynamics is qualitatively very similar to the single mode case (Grießer et al., 2010) shown in Fig. 11 of the previous section. One clearly recognizes the striking difference in the dynamical behavior for positive and negative values of $\delta_{C}$. While we have an instability in both cases, self-organization is only found for $\delta_{C}<0$.

c. Sympathetic self-ordering and cooling Self-organization and collective coherent light scattering into a high-finesse cavity in principle allows for trapping and cooling of any kind of polarizable particles. In practice, however, the required phase-space densities and laser intensities to initiate the ordering process are hard to achieve for atomic
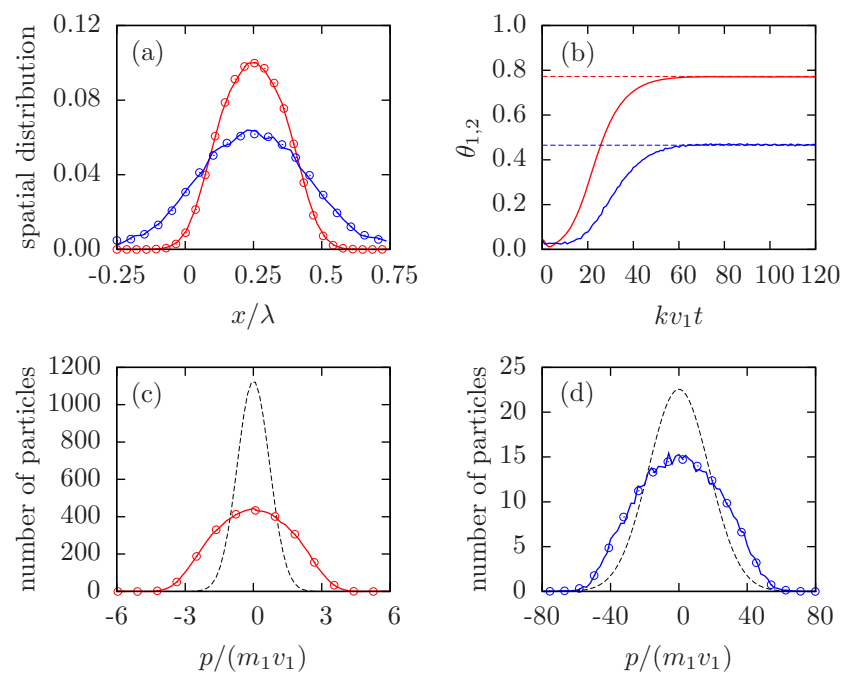

FIG. 18 (Color online) Simultaneous self-organization of two species. The system is started from a perturbed uniform state above the instability threshold, Eq. (39), in such a way that species one (two) itself would be pumped six times above (far below) the critical point. Figure (a) shows the position distributions in the final state, (c) and (d) the momentum distributions initially (dashed lines) and after self-organization (solid line). Solid lines depict the results of stochastic trajectory simulations for ensembles of particles as in Eq.(23b), while open circles show the predictions of the corresponding Vlasov model. Figure (b) shows the time evolution of the two order parameters $\theta_{1(2)}$ approaching theoretical steady-state values. Parameters are $N_{1}=10^{4}, N_{2}=500, m_{2}=10 m_{1}$, $k_{\mathrm{B}} T_{1}=10^{4} \hbar \kappa, k_{\mathrm{B}} T_{2}=2.5 \times 10^{5} \hbar \kappa, \eta_{1}=2.4 \kappa, \eta_{2}=27.4 \kappa$ and $\omega_{R}=10^{-2} \kappa$. From Grießer et al. (2011).

species, molecules or nanoparticles which cannot be efficiently optically precooled (Deachapunya et al., 2008; Lev et al., 2008). As an alternative approach, the selforganization threshold can be achieved by inserting different species simultaneously into an optical resonator.

The Vlasov-type model can be generalized to a dilute gas of various kinds of $N_{s}$ polarizable point particles of mass $m_{s}$ illuminated by a single transverse standing-wave laser field. For the threshold value, a condition analogous to Eq. (37) can be obtained (Grießer et al., 2011). The homogeneous distribution is unstable if and only if

$$
\sum_{s=1}^{S} \frac{\hbar N_{s} \eta_{s}^{2}}{k_{\mathrm{B}} T_{s}}\left(\operatorname{vp} \int_{-\infty}^{\infty} \frac{g_{s}^{\prime}(u)}{-2 u} d u\right)>\frac{\kappa^{2}+\delta_{C}^{2}}{\left|\delta_{C}\right|}
$$

where $k_{\mathrm{B}} T_{s}=m_{s} v_{s}^{2} / 2$. Note that the right-hand side of Eq. (39) depends only on cavity parameters, and all terms in the sum on the left-hand side are positive and proportional to the pump intensity. This guarantees that inserting any additional species into the cavity always increases the total light scattering rate and thus lowers the minimum power needed to start the self-organization process, regardless of temperature and polarizability or density of the additional particles. Moreover, the different 


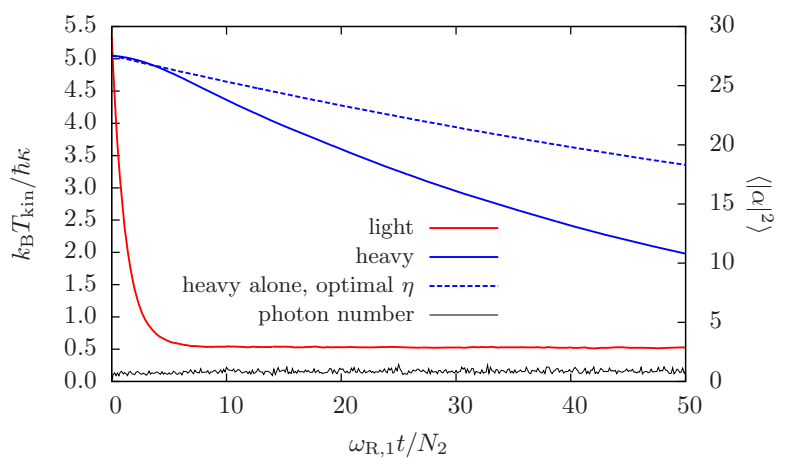

FIG. 19 (Color online) Sympathetic cavity cooling. Time evolution of the kinetic temperatures of a heavy and a light species. The blue dashed line represents the heavy particle alone and the blue solid line the enhanced cooling in the presence of a lighter species below self-organization threshold. Parameters are $m_{2}=200 m_{1}, N_{1}=200, N_{2}=200$, $\sqrt{N_{1}} \eta_{1}=134 \omega_{R}, \sqrt{N_{2}} \eta_{2}=134 \omega_{R}, \kappa=200 \omega_{R}$ and $\delta_{C}=-\kappa$. From Grießer et al. (2011).

species can be located at different regions within the cavity. Assisted self-organization of a species which, alone, would be pumped below threshold is shown in Fig. 18 .

Below the self-organization threshold cooling occurs thereby equalizing the stationary momentum distributions for all species. Fig. 19 exhibits the enhanced decay of the kinetic energy of the heavy particles in presence of cavity field and a cold species. Although the distributions get independent in stationary equilibrium, the cooling process itself involves energy exchange between different species. Thus if any of the species is cold or can be cooled by different means, the other components are sympathetically cooled in parallel.

\section{Non-equilibrium steady-state distributions}

Over longer time scales, diffusion has to be accounted for in terms of a nonlinear Fokker-Planck equation for the statistically averaged velocity distribution. This allows for the calculations of cooling time scales and the unique steady-state distribution.

a. Transverse pump configuration below threshold Below the instability threshold, Eq. (37), the mean spatial distribution $f$ is homogeneous, i.e., independent of $x$. The statistical fluctuations of the potential and the actual atomic distribution gets important. For the spatially averaged distribution, a lengthy calculation (Niedenzu et al., 2011) leads to a nonlinear Fokker-Planck equation for the velocity distribution $F(v, t)=\overline{f(x, v, t)}$,

$$
\frac{\partial}{\partial t} F+\frac{\partial}{\partial v}(A[F] F)=\frac{\partial}{\partial v}\left(B[F] \frac{\partial}{\partial v} F\right)
$$

with coefficients

$$
\begin{aligned}
& A[F]=\frac{2 \hbar k \delta_{C} \kappa \eta_{\mathrm{eff}}^{2}}{m} \frac{k v}{|D(i k v)|^{2}} \\
& B[F]=\frac{\hbar^{2} k^{2} \eta_{\mathrm{eff}}^{2} \kappa}{2 m^{2}} \frac{\kappa^{2}+\delta_{C}^{2}+k^{2} v^{2}}{|D(i k v)|^{2}} .
\end{aligned}
$$

These functionals depend on $\langle F\rangle$ via the dispersion relation

$$
\begin{aligned}
& D(s)=(s+\kappa)^{2}+\delta_{C}^{2}- \\
& -i \hbar k \delta_{C} \frac{N L \eta_{\mathrm{eff}}^{2}}{2 m} \int_{-\infty}^{\infty} d v\left(\frac{F^{\prime}(v)}{s+i k v}-\frac{F^{\prime}(v)}{s-i k v}\right),
\end{aligned}
$$

which encodes all cavity-mediated long-range particle interactions. Far below threshold the dispersion relation reduces to $D(i k v) \simeq(i k v+\kappa)^{2}+\delta_{C}^{2}$, which corresponds to the case of independent particles.

Steady-state solutions of Eq. (40) exist only for negative detuning $\delta_{C}<0$, where light scattering is accompanied by kinetic energy extraction from atomic motion. Below threshold one obtains non-thermal $q$-Gaussian velocity distribution functions (de Souza and Tsallis, 1997):

$$
F(v) \propto\left(1-(1-q) \frac{m v^{2}}{2 k_{\mathrm{B}} T}\right)^{\frac{1}{1-q}},
$$

with $q=1+\omega_{R} /\left|\delta_{C}\right|$ and the effective temperature

$$
k_{\mathrm{B}} T=\hbar \frac{\kappa^{2}+\delta_{C}^{2}}{4\left|\delta_{C}\right|} \geq \frac{\hbar \kappa}{2} .
$$

The minimum temperature is reached for $\delta_{C}=-\kappa$. The magnitude of the detuning $\left|\delta_{C}\right| / \omega_{R}$ determines the shape of the distribution. For $\left|\delta_{C}\right|=\omega_{R}$, it is a Lorentzian distribution, whereas for $\left|\delta_{C}\right| / \omega_{R} \rightarrow \infty$, i.e. $q \rightarrow 1$, it converges to a Gaussian distribution with kinetic temperature $k_{\mathrm{B}} T_{\text {kin }}=m\left\langle v^{2}\right\rangle$.

Inserting the steady-state $q$-Gaussian (43) distribution into the threshold condition (37) gives a self-consistent stability criterion. As a result, the homogeneous distribution is stable only if

$$
\sqrt{N} \eta_{\mathrm{eff}} \leq \kappa \sqrt{\frac{2}{3-q}}
$$

where the equality is reached for $\delta_{C}=-\kappa$, i.e., for optimum cavity cooling given by Eq. (14). The stability criterion can be rewritten in the intuitive form

$$
N\left|U_{0}\right| V_{p} \leq \kappa^{2},
$$

where $V_{p}=\Omega^{2} / \Delta_{A}$ is the optical potential depth created by the pump laser, and $N U_{0}$ is the total dispersive shift of the cavity resonance. Note that even if the initial temperature is too high for the homogeneous distribution to be unstable, cavity cooling induced self-organization is possible. 


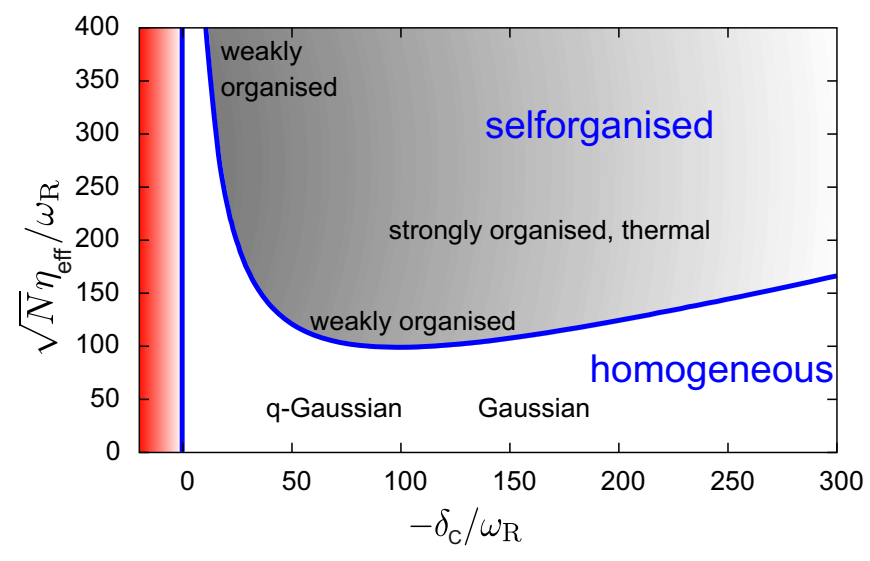

FIG. 20 (Color online) Schematic view of the phase diagram in the weak-coupling limit $\left(N\left|U_{0}\right| \ll \kappa\right)$ for $\kappa=$ $100 \omega_{R}$. Equilibrium solutions exist only for $\delta_{C}<-\omega_{R} / 2$, a Lorentzian steady-state velocity distribution is realized for the case $\left|\delta_{C}\right|=\omega_{R}$. For large negative values of the detuning $\delta_{C}$, strongly organized equilibrium solutions exist already for pump strengths slightly above the critical value. Adapted from Niedenzu et al. (2011)

b. Transverse pump configuration above threshold Above the self-organization threshold, the inhomogeneous spatial distribution can still be derived from a Fokker-Planck equation similar to Eq. (40) by using action-angle variables (Chavanis, 2007; Luciani and Pellat, 1987). In the limit of deep trapping and a harmonic approximation for the potential, the steady state is a thermal distribution with a temperature depending both on the effective trap frequency $\omega_{0}$ and on the cavity linewidth $\kappa$,

$$
k_{\mathrm{B}} T=\hbar \frac{\kappa^{2}+\delta_{C}^{2}+4 \omega_{0}^{2}}{4\left|\delta_{C}\right|} \stackrel{\delta_{C}=-\omega_{0}}{\approx} \hbar \omega_{0} .
$$

The effective trap frequency $\omega_{0}$ can be approximated by

$$
\omega_{0}^{2} \simeq \sqrt{N} \eta_{\mathrm{eff}} \omega_{R}\left(\frac{\eta_{\mathrm{eff}}}{\eta_{\mathrm{eff}, \mathrm{c}}}+\sqrt{\frac{\eta_{\mathrm{eff}}^{2}}{\eta_{\mathrm{eff}, \mathrm{c}}^{2}}-1}\right)
$$

which is valid in the regime $\left|\delta_{C}\right| \gg \omega_{R}$ with $\eta_{\text {eff,c given }}$ by the equality of Eq. (45). As the temperature depends explicitly on the pump strength, a stronger pump laser beam results in more confined particles with increased kinetic energy. The system has the interesting property that the more particles we add, the deeper the optical potential gets, which shows analogy to self-gravitating systems (Posch and Thirring, 2005).

Finally, putting all this together one obtains the selfconsistent phase diagram for self-organization which accounts for cavity cooling, see Fig. 20.

\section{QUANTUM GASES IN OPTICAL CAVITIES}

Quantum gases are considered as ideal model systems to study quantum many-body phenomena under well-controlled experimental conditions. The possibilities which arise from loading ultracold atomic ensembles of different particle statistics into various optical potential landscapes and to tune the strength of the contact atomatom interaction, make these system well suited for quantum information and simulation research (Bloch et al., 2008). The merger of the field of ultracold gases with that of cavity QED provides a set of additional possibilities. Cavity-mediated atom-atom interactions can be tailored by choosing different resonator and pump geometries and give rise to novel quantum phases. Closely related, the atomic back-action upon the cavity-generated lattice potentials can be significant, which paves the way to study phonon or soft-condensed matter physics with ultracold gases (Lewenstein et al., 2007). Further, coherent scattering into the cavity field can be used for non-destructive and real-time probing of different many-body phases.

The coupling between a Bose-Einstein condensate and an optical cavity is conceptually fundamental since a single mode of a matter wave field interacts with a single mode of the light field: as all atoms occupy the same motional quantum state they couple identically to the optical cavity field. This situation can substantially reduce the number of degrees of freedom necessary to describe the system. Therefore the experimental situation can often be almost perfectly described by fundamental Hamiltonians of matter-light interaction. These include the Tavis-Cummings or Dicke model, as well as the generic model for cavity optomechanics.

\section{A. Experimental realizations}

Experimentally, there have been different approaches to realize and study Bose-Einstein condensates or bosonic atomic ensembles close to quantum degeneracy in optical high-finesse cavities (Brennecke et al., 2007; Colombe et al., 2007; Gupta et al., 2007; Purdy et al., 2010; Slama et al., 2007a). So far, all groups used ${ }^{87} \mathrm{Rb}$ atoms. In the Tübingen group (Slama et al., 2007a) a Bose-Einstein condensate was loaded for the first time into a ring cavity with large mode volume using magnetic trapping and transport. This experiment extended prior work on the collective atomic recoil laser (see Sect. III.B.2) with lasercooled atoms (Cube et al., 2004; Kruse et al., 2003a) into the ultracold regime.

Loading ultracold quantum gases or Bose-Einstein condensates into ultra-high finesse optical cavities of small mode volume, which operate in the single-atom strong coupling regime of cavity QED, has been achieved by applying different concepts. The Berkeley group (Gupta et al., 2007) prepared an ultracold gas of up to $10^{5}$ atoms 
(a)

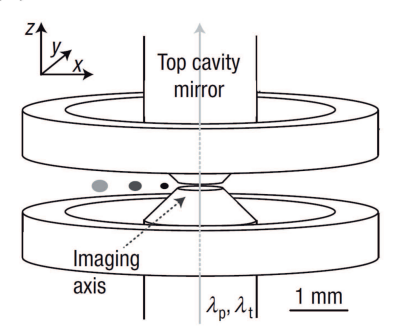

(c)
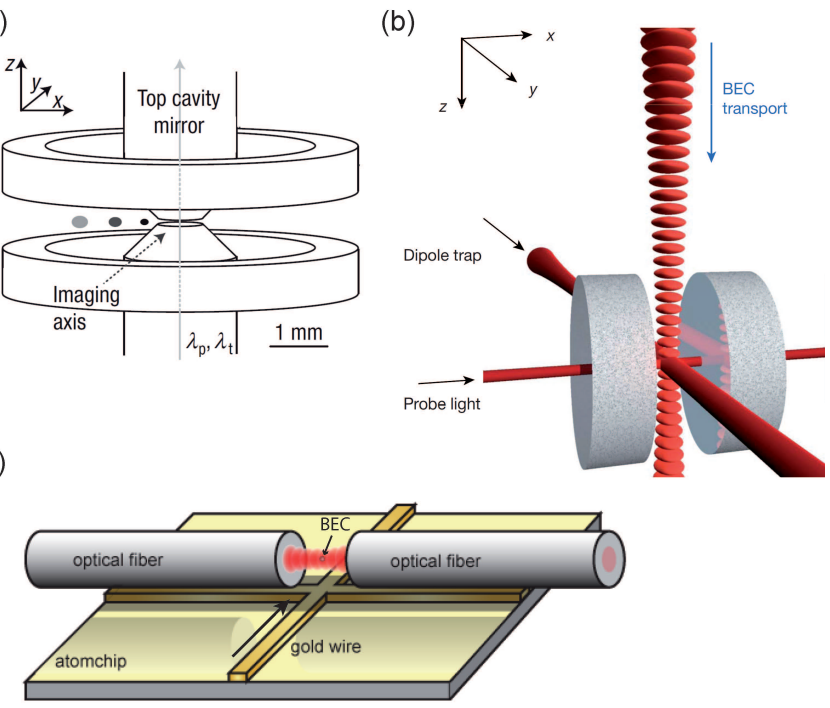

(d)

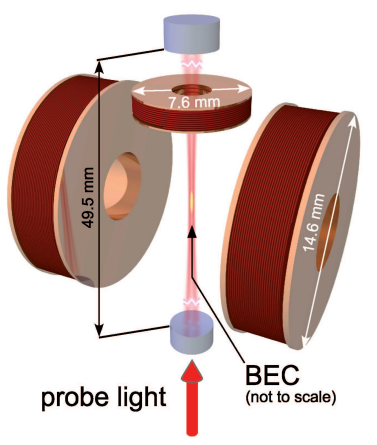

FIG. 21 (Color online) Different experimental schemes for preparing ultracold atoms and Bose-Einstein condensates inside high-finesse optical Fabry-Pérot resonators. (a) Ultracold atoms are prepared in a magnetic trap, formed using electromagnets coaxial with the vertically oriented high-finesse cavity (length $=194 \mu \mathrm{m}$ ) and delivered along the $x$-axis towards the cavity center. Once overlapping with the cavity mode, the atoms are loaded into a deep intracavity lattice potential provided by a far-detuned cavity pump field. Adapted from Murch et al. (2008). (b) Ultracold atoms, prepared in a magnetic trap placed above the optical resonator, are loaded into a vertically oriented optical lattice potential and transported into the cavity by controlled frequency chirping the counter-propagating laser beams. Once in the cavity (length $=176 \mu \mathrm{m}$ ), the atoms are loaded into a crossedbeam harmonic dipole trap where Bose-Einstein condensation is achieved. From Brennecke et al. (2007). (c) A BoseEinstein condensate is prepared in an atom-chip-based magnetic trap and positioned afterwards with subwavelength precision in the mode of a fibre-based Farby-Pérot cavity which has a length of $39 \mu \mathrm{m}$. Figure courtesy of J. Reichel. (d) A magnetic QUIC trap is used to prepare and to transfer a Bose-Einstein condensate into the field of a vertically oriented Fabry-Pérot resonator with a length of $5 \mathrm{~cm}$. Figure courtesy of A. Hemmerich. within an ultra-high finesse Fabry-Pérot resonator by loading it into a vertically oriented, deep intracavity optical lattice potential, see Fig. 21a. A cavity with similar parameters was used in the approach of the Zürich group (Brennecke et al., 2007). Here, Bose-Einstein condensates of typically $2 \times 10^{5}$ atoms were transported into the mode volume of the optical cavity using an optical elevator formed by two counter-propagating laser beams with controlled frequency difference, see Fig. 21b. The Paris group (Colombe et al., 2007) used an atomic chip to produce Bose-Einstein condensates of up to 3000 atoms and control its position on a sub-wavelength scales within a novel type of fibre-based Fabry-Pérot cavity with high mirror curvature and reduced mode volume, see Fig. 21c. Also the Berkeley group (Purdy et al., 2010) achieved sub-wavelength positioning of Bose-Einstein condensates of a few thousands of atoms inside a conventional smallvolume high-finesse optical cavity using an atomic chip.

A novel BEC-cavity system operating in an interesting and so far unexplored parameter regime was presented recently by the Hamburg group (Wolke et al., 2012). Here, Bose-Einstein condensates of typical $2 \times 10^{5}$ atoms are prepared and transferred magnetically into the field of a $5 \mathrm{~cm}$ long near-concentric Fabry-Pérot resonator resulting in a large single-atom cooperative $\mathcal{C} \gg 1$ and a very narrow cavity bandwidth on the order of the recoil frequency $\omega_{R}$, see Fig. 21d.

The lowest electronic excitation spectrum of degenerate and non-degenerate atomic samples strongly coupled to the cavity field has been studied by Brennecke et al. (2007) and Colombe et al. (2007). The presence of $N$ atoms which collectively couple to the cavity field results in an enhanced collective coupling which scales as $\sqrt{N}$. A correspondingly large vacuum Rabi-splitting was measured in the experiments (Brennecke et al., 2007; Colombe et al., 2007). The energy spectrum of a coupled BEC-cavity system together with the square-root dependence of the energy splitting on the atom number are shown in Fig. 22.

The electronic excitation spectrum is sensitive to the effective number of atoms coupled maximally to the cavity mode, i.e. it depends on the density distribution of the atoms integrated over the cavity mode profile. However, the above described measurements of the electronic excitation spectrum have an energy resolution given by the excited state and cavity lifetimes, which is too large to probe the low-energy excitations of the external degree of freedom of a Bose-Einstein condensate. Probing quantum statistics and quantum correlations in atomic manybody states using the dispersive coupling to far-detuned laser and cavity fields is discussed in Sect. IV.E.2. 


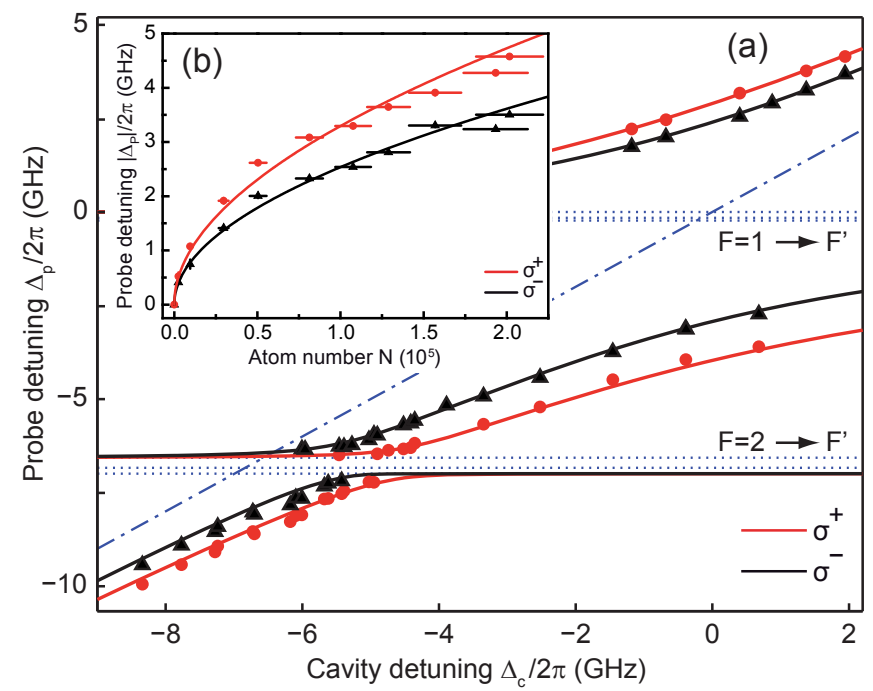

FIG. 22 (Color online) Collective vacuum Rabi-splitting of a coupled BEC-cavity system. The displayed data was obtained by cavity transmission spectroscopy using a weak probe laser beam (Brennecke et al., 2007). The detuning of the probe beam with respect to the frequency $\omega_{A}$ of the $|F=1\rangle \rightarrow$ $\left|F^{\prime}=1\right\rangle$ transition of the $\mathrm{D}_{2}$ line of ${ }^{87} \mathrm{Rb}$ is denoted by $\Delta_{p}$. Two orthogonal circular polarizations of the transmitted light were recorded and are displayed as red circles $\left(\sigma^{+}\right)$and black triangles $\left(\sigma^{-}\right)$. (a) Position of the probed resonances as a function of the detuning $\Delta_{c}$ between the empty cavity resonance and the atomic transition frequency $\omega_{A}$ for $2.2 \times 10^{5}$ atoms. Bare atomic resonances are shown as dotted lines, whereas the empty cavity resonance of the $\mathrm{TEM}_{00}$ is plotted as a dashed-dotted line. The solid lines are the result of a theoretical model including the influence of higher-order cavity modes. (b) Shift of the lower resonance of the coupled BEC-cavity system from the bare atomic resonance as a function of atom number for $\Delta_{c}=0$. The solid lines are fits of the square-root dependence on the atom number $N$. Adapted from Brennecke et al. (2007).

\section{B. Theoretical description}

This section provides the theoretical basis for a quantum many-body description of a coupled and laser-driven BEC-cavity system at zero temperature. For simplicity, we consider two different pump laser fields with equal frequency $\omega$ which propagate along and transverse to the axis of a Fabry-Pérot resonator. A similar many-body description for the case of a BEC in a driven ring-cavity has been presented e.g. in Moore et al. (1999). The Hamiltonian is composed of an atomic, a cavity and an atom-field interaction part

$$
H=H_{A}+H_{C}+H_{A C} .
$$

In the following, we will assume a sufficiently large detuning of the cavity frequency $\omega_{C}$ and the pump laser frequency $\omega$ from the atomic transition frequency $\omega_{A}$, so that the atom-field interaction is of purely dispersive na- ture, see Sec. II.A.2. In this case all excited states can be adiabatically eliminated and the atom resides most of the time in its electronic ground state. Correspondingly, the motional degree of freedom is captured by a scalar matter-wave field operator $\Psi(\mathbf{r})$.

The atomic many-body Hamiltonian is given by

$$
H_{A}=\int d^{3} \mathbf{r} \Psi^{\dagger}(\mathbf{r})\left[\mathcal{H}^{(1)}+\frac{u}{2} \Psi^{\dagger}(\mathbf{r}) \Psi(\mathbf{r})\right] \Psi(\mathbf{r}),
$$

where $u=4 \pi \hbar^{2} a_{s} / m$ denotes the strength of the short-range s-wave collisions with scattering length $a_{s}$ (Pitaevskii and Stringari, 2003). Here, the single-atom Hamiltonian

$$
\mathcal{H}^{(1)}=\frac{\mathbf{p}^{2}}{2 m}+V_{\mathrm{cl}}(\mathbf{r}),
$$

includes an external trapping potential $V_{\mathrm{cl}}(\mathbf{r})$ which also incorporates the potential caused by the transverse pump laser field.

The dynamics of a single, coherently laser-driven cavity mode with mode function $\cos (k x)$ and resonance frequency $\omega_{C}$ is described in a frame rotating at the pump laser frequency $\omega$ by the Hamiltonian

$$
H_{C}=-\hbar \Delta_{C} a^{\dagger} a+i \hbar \eta\left(a^{\dagger}-a\right) .
$$

As before, the detuning between the pump laser frequency and the cavity resonance frequency is denoted by $\Delta_{C}=\omega-\omega_{C}$. The generalization to multimode cavities will be discussed in Sec. IV.D.3.

The dispersive interaction between the pump and cavity radiation fields and the atoms reads (in the frame rotating at $\omega)$

$$
\begin{aligned}
H_{A C}= & \int d^{3} \mathbf{r} \Psi^{\dagger}(\mathbf{r})\left[\hbar U_{0} \cos ^{2}(k x) a^{\dagger} a\right. \\
& \left.+\hbar \eta_{\text {eff }} \cos (k x) \cos (k z)\left(a^{\dagger}+a\right)\right] \Psi(\mathbf{r})
\end{aligned}
$$

The first term arises from the absorption and stimulated emission of cavity photons, with $U_{0}=\frac{g^{2}}{\Delta_{A}}$ denoting the maximum atomic light-shift for a single intracavity photon. As before, $g$ denotes the maximum atom-cavity coupling strength and $\Delta_{A}=\omega-\omega_{A}$ the pump-atom detuning. The second term corresponds to the coherent redistribution of photons between the standing-wave transverse pump laser (with mode function $\cos (k z)$ ) and the cavity field. The maximum scattering rate for a single atom is given by the two-photon (vacuum) Rabi frequency $\eta_{\mathrm{eff}}=\frac{\Omega g}{\Delta_{A}}$, where $\Omega$ is the Rabi frequency of the transverse pump laser. Both interaction terms in Eq. (53) can be viewed as a four-wave mixing of light and matter wave fields (Rolston and Phillips, 2002).

The system is subject to dissipation due to photon leakage through the cavity mirrors. The corresponding irreversible evolution can be modeled by the Liouville 
terms in the master equation, Eq. (5a), or, equivalently, by a Heisenberg-Langevin equation (Gardiner and Zoller, 2004) for the cavity field operator $a$,

$$
\frac{d}{d t} a=-i[a, H]-\kappa a+\xi,
$$

with cavity field decay rate $\kappa{ }^{1}$ The Gaussian noise operator $\xi$ maintains the commutation relation for the photon operators in the presence of cavity decay. In the optical domain, the temperature of the bath of electromagnetic field modes can be set to zero. Correspondingly, $\xi$ has zero mean value and the only non-vanishing correlation function reads

$$
\left\langle\xi(t) \xi^{\dagger}\left(t^{\prime}\right)\right\rangle=2 \kappa \delta\left(t-t^{\prime}\right),
$$

according to the fluctuation-dissipation theorem. Further possible dissipation channels can, for example, act directly on the atomic cloud.

As the cavity field mediates a global coupling among all atoms, a mean-field approach is well suited to solve the above set of equations (Horak et al., 2000; Horak and Ritsch, 2001a; Nagy et al., 2008). The mean-field description assumes the presence of a macroscopically populated matter wave field $\varphi(\mathbf{r})=\langle\Psi(\mathbf{r})\rangle$ (condensate wavefunction) and a coherent cavity field with amplitude $\alpha=\langle a\rangle$ which can be separated from the quantum fluctuations according to

$$
\begin{aligned}
a & \rightarrow \alpha+\delta a \\
\Psi(\mathbf{r}) & \rightarrow \sqrt{N_{c}} \varphi(\mathbf{r})+\delta \Psi(\mathbf{r}) .
\end{aligned}
$$

Here, $N_{c}$ denotes the number of condensate atoms with $\varphi(\mathbf{r})$ being normalized to 1. Quantum fluctuations are assumed to be small and their mean values vanish by definition, i.e. $\langle\delta a\rangle=0$ and $\langle\delta \Psi(\mathbf{r})\rangle=0$. The dynamical equations of motion resulting from Eqs. (56) contain a hierarchy of terms according to the different powers of the fluctuation operators. To zeroth order in the fluctuations, one obtains a Gross-Pitaevskii-type equation for the condensate wavefunction $\varphi(\mathbf{r}, t)$ coupled to an ordinary differential equation for $\alpha(t)$ :

$$
\begin{aligned}
& i \hbar \frac{\partial}{\partial t} \varphi(\mathbf{r}, t)=[-\frac{\hbar^{2} \nabla^{2}}{2 m}+V_{0}(\mathbf{r})+N_{c} u|\varphi(\mathbf{r}, t)|^{2} \\
&+\hbar U_{0}|\alpha(t)|^{2} \cos ^{2}(k x) \\
&\left.+2 \hbar \eta_{\text {eff }} \operatorname{Re}\{\alpha(t)\} \cos (k x) \cos (k z)\right] \varphi(\mathbf{r}, t)
\end{aligned}
$$

\footnotetext{
${ }^{1}$ It is important to note that the original problem is intrinsically time-dependent because of the external laser driving, although this time dependence has been formally eliminated by going into a rotating frame. Nevertheless, the coupling to the reservoir occurs at the high, optical frequency range and thus the simple form of the loss description can be used irrespective of the lowfrequency dynamics imposed by the effective Hamiltonian.
}

$$
\begin{aligned}
i \frac{\partial}{\partial t} \alpha(t)=\left[-\Delta_{C}+\right. & \left.N_{c} U_{0}\left\langle\cos ^{2}(k x)\right\rangle-i \kappa\right] \alpha(t)+i \eta \\
& +N_{c} \eta_{\mathrm{eff}}\langle\cos (k x) \cos (k z)\rangle,
\end{aligned}
$$

where we used the notation $\langle f(\mathbf{r})\rangle=\int d^{3} r f(\mathbf{r})|\varphi(\mathbf{r}, t)|^{2}$. The Gross-Pitaevskii equation contains potential-like terms which depend on the amplitude $\alpha$ and intensity $|\alpha|^{2}$ of the cavity field, and express the mechanical effect of the cavity light upon the atoms. The dynamics of the cavity field involves spatial averages over the condensate density distribution. Because of cavity decay, the time evolution leads to a self-consistent stationary solution for the mean fields, which usually is obtained only numerically (Nagy et al., 2008).

For a given condensate wave function and coherent cavity field amplitude in steady state, the quantum fluctuations to leading order form a linear system, which provides the energy spectrum of excitations. With the notation $\mathbf{R}=\left[\delta a, \delta a^{\dagger}, \delta \Psi(\mathbf{r}), \delta \Psi^{\dagger}(\mathbf{r})\right]$, the time evolution of the fluctuation operators takes the compact form

$$
\frac{\partial}{\partial t} \mathbf{R}=\mathbf{M R}+\boldsymbol{\Xi}
$$

where $\mathbf{M}$ is the linear stability matrix of the mean field solution (Nagy et al., 2008), and the term $\boldsymbol{\Xi}=\left[\xi, \xi^{\dagger}, 0,0\right]$ accounts for the quantum input noise of the cavity field. In general, the matrix $\mathbf{M}$ is non-normal, i.e. it does not commute with its hermitian adjoint. Therefore it has different left and right eigenvectors, denoted by $\mathbf{l}^{(k)}$ and $\mathbf{r}^{(k)}$, that form a bi-orthogonal system with scalar product $\left(\mathbf{l}^{(k)}, \mathbf{r}^{(l)}\right)=\delta_{k, l}$. The decoupled quasi-normal excitation modes defined by $\rho_{k}=\left(\mathbf{l}^{(k)}, \mathbf{R}\right)$ are mixed excitations of the photon and the matter wave fields.

The spectrum of excitations was analyzed first from a cavity cooling point of view in the cavity pumping geometry $\left(\eta \neq 0, \eta_{\text {eff }}=0\right)$. The imaginary part of the spectrum revealed that excitations of the ultracold atomic gas can be damped through the cavity loss channel (Gardiner et al., 2001; Horak and Ritsch, 2001a), provided the decay rate $\kappa$ is on the order of the recoil frequency $\omega_{R}$. The excitation spectrum was used in further studies to describe critical phenomena, such as the dispersive optical bistability in the cavity pumping geometry (Szirmai et al., 2010) and the self-organization phase transition (Kónya et al., 2011; Nagy et al., 2008) in the atom pumping geometry $\left(\eta_{\text {eff }} \neq 0, \eta=0\right)$, see Sec. IV.C and Sec. IV.D.

In the stable regime, second-order correlation functions can be derived from Eq. (58). Importantly, there can be a non-vanishing population of the atomic excited modes, $\left\langle\delta \Psi^{\dagger}(\mathbf{r}) \delta \Psi(\mathbf{r})\right\rangle \neq 0$, even at zero temperature. This quantum depletion of the condensate is independent of collisional interactions, which are known to cause a finite population of the Bogoliubov modes (Pitaevskii and Stringari, 2003). At variance, here the condensate depletion arises from the cavity-mediated atom-atom interactions as well as the dissipative process associated 
with cavity decay. The quantum noise accompanying the photon loss process couples into the atomic system and excites atoms out of the condensate mode. Formally, it stems from the term containing the photon creation operator $a^{\dagger}$ in the equations of motion of $\Psi(\mathbf{r})$. This noise amplification mechanism is analogous to the Petermann excess noise factor in lasers with unstable cavities (Grangier and Poizat, 1998). It was shown by Szirmai et al. (2009) that a depletion on the order of $\sqrt{\Delta_{C}^{2}+\kappa^{2}} / \omega_{R}$ can be expected rather independently of the atom-field coupling, even for $U_{0} \rightarrow 0$ and $\eta_{\text {eff }}=0$. It is a signature of the global coupling that the quantum depletion is independent of the total atom number $N$. In most of the experiments with linear cavities, the ratio $\kappa / \omega_{R}$ is large $\left(\sim 10^{3}\right)$. Since cavity decay can also be interpreted as a continuous weak measurement of the cavity photon number, the depletion can be attributed to quantum back-action upon the atomic many-body state, as discussed in Sec. IV.C.3. It is also interesting to note that the second-order correlation functions reveal an entanglement between the matter-wave and the cavity field modes (Szirmai et al., 2010).

\section{Cavity opto-mechanics with ultracold atomic ensembles}

In this section we focus on the dispersive interaction between the collective motion of a quantum gas and a single-mode Fabry-Pérot cavity, which is coherently driven with amplitude $\eta$ by a laser field at frequency $\omega$. In this case the dispersive matter-light interaction, (53), is given by

$$
H_{A C}=\int d^{3} \mathbf{r} \Psi^{\dagger}(\mathbf{r})\left[\hbar U_{0} \cos ^{2}(k x) a^{\dagger} a\right] \Psi(\mathbf{r})
$$

On the one hand, the atomic medium experiences a periodic potential, whose depth is proportional to the intracavity photon number $a^{\dagger} a$. The potential depth for a single cavity photon is $U_{0}=g^{2} / \Delta_{A}$ and can be tuned in the experiment via the detuning $\Delta_{A}$ between the cavity pump frequency $\omega$ and the atomic transition frequency. On the other hand, the atom-light interaction causes a dispersive shift of the empty cavity frequency, which is determined by the spatial overlap between the atomic density $\Psi^{\dagger}(\mathbf{r}) \Psi(\mathbf{r})$ and the cavity intensity mode function $\cos ^{2}(k x)$. A change in the atomic density distribution caused by the intracavity dipole force can therefore dynamically act back on the intracavity field intensity by shifting the cavity resonance with respect to the driving field.

In general, the interplay of these two effects results in a highly nonlinear evolution of the coupled atoms-cavity system. For certain limiting situations, however, the system can effectively be described in the framework of cavity optomechanics (Kippenberg and Vahala, 2008), which studies the radiation-pressure interaction between a harmonically suspended mechanical element and the field inside an electromagnetic resonator. In a frame rotating at $\omega$ this is described by the generic cavity optomechanics Hamiltonian

$$
H_{\mathrm{OM}}=\hbar \omega_{m} c^{\dagger} c-\hbar\left(\delta_{C}-G X\right) a^{\dagger} a+i \hbar \eta\left(a^{\dagger}-a\right)
$$

where $c^{\dagger}$ and $c$ denote creation and annihilation operators of the mechanical oscillator at frequency $\omega_{m}$. The mechanical element couples via its position quadrature $X=\left(c+c^{\dagger}\right) / \sqrt{2}$ with coupling strength $G$ to the intracavity photon number $a^{\dagger} a$. The detuning between the driving laser and the cavity resonance frequency for zero displacement $X$ is denoted by $\delta_{C}$. Configurations in which the harmonic oscillator couples quadratically in $X$ to the cavity field have recently been realized (Purdy et al., 2010; Thompson et al., 2008). This offers the possibility to detect phonon Fock states of the mechanical element and to prepare squeezed states of the mechanical oscillator or the optical output field.

\section{Experimental realizations}

Particular experimental situations allow to realize the optomechanics Hamiltonian Eq. (60) with an atomic ensemble dispersively coupled to the field inside an optical cavity. This relies on the fact, that the cavity field affects and senses predominantly a single collective motional mode, which matches the spatial cavity mode profile and plays the role of the harmonically suspended mechanical element. Two different approaches for realizing cavity optomechanics with ultracold atoms have so far been realized experimentally.

a. Collective center-of-mass motion in the Lamb-Dicke regime In experiments performed by the Berkeley group (Gupta et al., 2007; Murch et al., 2008), ultracold atoms are loaded into the lowest band of a far-detuned intracavity lattice potential, forming a stack of hundreds of tightly confined atom clouds (see Fig. 23). Each atom cloud is harmonically suspended with oscillation frequency $\omega_{m}$ and extends along the cavity axis by only a fraction of the optical wavelength, thus realizing the Lamb-Dicke regime. A cavity mode, whose periodicity differs from that of the trapping lattice potential, couples strongly to a single collective center-of-mass mode of the atomic stack. All remaining collective modes decouple from the cavity field and can be considered as a heat bath to which the distinguished collective mode is only weakly coupled via e.g. collisional atom-atom interactions. The system realizes the linear optomechanics Hamiltonian Eq. (60), with the optomechanical coupling strength being given by $G=\sqrt{N_{\mathrm{eff}}} k U_{0} X_{\mathrm{ho}}$. Here, $k$ is the cavity wave vector, $X_{\text {ho }}=\sqrt{\hbar / 2 m \omega_{m}}$ denotes the harmonic oscillator 


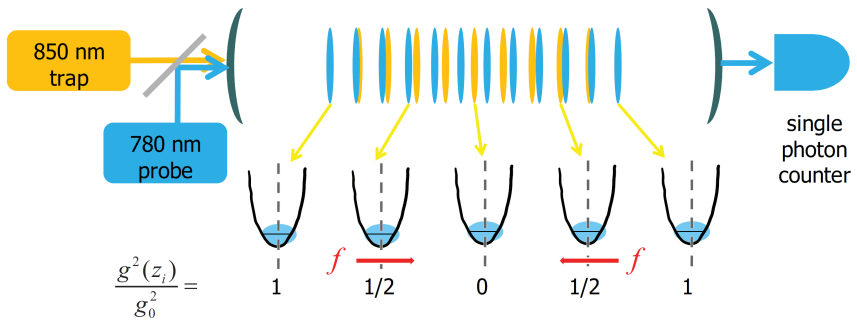

FIG. 23 (Color online) Scheme for cavity optomechanics with ultracold atoms confined in the Lamb-Dicke regime. A highfinesse cavity supports two longitudinal modes: one with wavelength of about $780 \mathrm{~nm}$ that is near the $D_{2}$ line of ${ }^{87} \mathrm{Rb}$, and another with wavelength of about $850 \mathrm{~nm}$. The latter produces a one-dimensional optical lattice potential, with trap minima indicated in orange, in which ultracold ${ }^{87} \mathrm{Rb}$ atoms are confined within the lowest vibrational band. The atomic clouds induce, depending on their trapping position $z_{i}$, dispersive frequency shifts on the $780 \mathrm{~nm}$ cavity resonance, as shown in the bottom line. In turn, the cavity field exerts a position dependent force $f$, as indicated by the red arrows. In the Lamb-Dicke regime, the collective atoms-cavity interaction reduces to the generic optomechanics Hamiltonian wherein a single collective mode of harmonic motion linearly couples to the cavity field. From Botter et al. (2009).

length with atomic mass $m$, and $N_{\text {eff }} \approx N / 2$ with total atom number $N$.

The quadratic coupling regime of optomechanics with ultracold atoms was realized in an atom-chip-based setup (Purdy et al., 2010) which allows for subwavelength positioning of a tightly confined ultracold ensemble. By preparing as low as two atomic clouds, tightly confined at adjacent lattice sites of a far-detuned intracavity lattice potential, and controlling their center-of-mass position along the cavity axis both linear and quadratic optomechanical coupling can be realized, providing an atoms-based realization of the "membran-in-the-middle" approach (Thompson et al., 2008).

b. Collective density oscillations in a Bose-Einstein condensate A different route to realize linear cavity optomechanics with an ultracold atomic ensemble was experimentally explored in the Zürich group (Brennecke et al., 2008). A Bose-Einstein condensate of typically $10^{5}$ atoms is prepared in an external harmonic trapping potential, extending over several periods of the cavity standingwave mode structure (see Fig. 24). In contrast to the Lamb-Dicke regime considered before, here a momentum picture is more appropriate. Initially, all condensate atoms are prepared - relative to the recoil momentum $\hbar k$ - in the zero-momentum state $|p=0\rangle$. The dispersive interaction with the cavity field diffracts atoms into the symmetric superposition of momentum states $| \pm 2 \hbar k\rangle$ along the cavity axis. Matter-wave interference between the macroscopically occupied zero-momentum component and the recoiling component results in a spatial modulation of the condensate density with periodicity $\lambda / 2$, which oscillates in time at the frequency $4 \omega_{R}=2 \hbar k^{2} / m$. As long as diffraction into higher-order momentum modes can be neglected, the dynamics of the coupled system is again captured by the simple optomechanics Hamiltonian Eq. (60). Here, collective excitations of the recoiling momentum mode play the role of phonon excitations of a mechanical mode with oscillation frequency $\omega_{m}=4 \omega_{R}$. The coupling rate, $G=\sqrt{N} U_{0} / 2$, again scales with the square root of the atoms number, indicating the collective nature of the atom-light interaction. The realization of an optomechanical system employing the collective motion of a Bose-Einstein condensate triggered subsequent theoretical studies along this direction (Chen et al., 2011, 2010; De Chiara et al., 2011; Zhang et al., 2009).
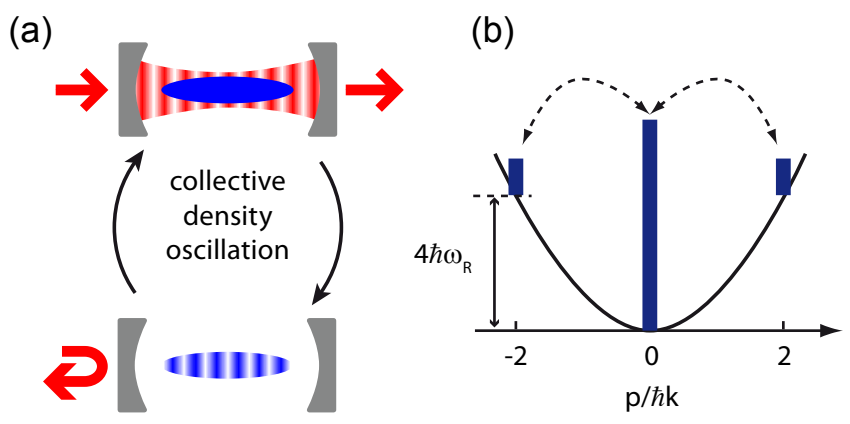

FIG. 24 (Color online) Cavity-optomechanics with a weakly confined Bose-Einstein condensate dispersively coupled to the field of an optical high-finesse resonator. (a) A collective density excitation of the condensate (blue) with periodicity $\lambda / 2=\pi / k$ acts as a mechanical oscillator with oscillation frequency $4 \omega_{R}$. Optomechanical coupling to the cavity field is provided by the dependence of the optical path length on the atomic density distribution within the spatially periodic cavity mode structure. (b) Condensate atoms initially prepared close to zero momentum, $p=0$, are scattered off the intracavity lattice potential into the symmetric superposition of states with momentum $p= \pm 2 \hbar k$. Matter-wave interference with the macroscopic zero-momentum component results in a harmonic density oscillation evolving at frequency $4 \omega_{R}$. Adapted from Brennecke et al. (2008).

Further realizations of cavity optomechanics with atomic ensembles have been proposed theoretically by dispersively coupling a quantum-degenerate Fermi gas (Kanamoto and Meystre, 2010) or the internal spindegrees of freedom of a quantum gas (Brahms and Kurn, 2010; Jing et al., 2011) to the field of an optical cavity. The latter system has been shown to exhibit a formal analogy with a torsional oscillator coupled quadratically to the cavity mode. It provides an ideal nondestructive tool for the control of quantum spin dynamics, and was proposed to resolve the quantum regime of an antiferromagnetic spin-1 condensate. 
The realization of an optomechanical system using ultracold atoms offers direct access to the quantum regime of cavity optomechanics. In contrast to solid-state realizations of optomechanics, evaporative cooling techniques available for atomic gases allow for a natural and very pure preparation of the mechanical oscillator mode in its quantum ground state. Correspondingly, these systems pave the way to directly study quantum effects of the optomechanical interaction (Brahms et al., 2012; Brooks et al., 2012; Murch et al., 2008).

The easy tunability of system parameters like e.g. the mechanical oscillator frequency $\omega_{m}$ (via the external confining potential), the optomechanical coupling strength $G$ (via the atom number or the pump-atom detuning) or the initial temperature of the mechanical oscillator allows to explore the transition between different regimes of optomechanics. Most important, the coupling strengths $G$ achievable with atomic systems open access to the 'granular' regime of optomechanics (Ludwig et al., 2008; Murch et al., 2008), where single excitations in either of the two subsystems have a non-negligible effect upon the dynamics of the other. This can be measured by the granularity (or quantum) parameter which is defined as $\zeta=G / \kappa$. For $\zeta=1$, already a single excitation of the mechanical mode shifts the cavity resonance by half its linewidth, and already a single photon entering the cavity imparts one excitation quantum in the mechanical oscillator. In future research, this might allow the generation and detection of quantum correlations between the mechanical and light degrees of freedom. Further research possibilities based on atoms-based realizations of optomechanics, are given by possible implementations of precision sensors of forces, or devices to manipulate light fields on a quantum level.

\section{Nonlinear dynamics and bistability for low photon number}

The optomechanical interaction, Eq. (60), being intrinsically nonlinear gives rise to dispersive optical bistability and nonlinear dynamics of the coupled system. Optical bistability (Lugiato, 1984), a well studied phenomenon in nonlinear optics, refers to the co-existence of two stable steady-state solutions when e.g. driving an optical cavity filled with a medium whose refractive index depends on the light intensity. In typical nonlinear Kerr media and solid-state realizations of optomechanics, the occurrence of bistability typically requires large intracavity power in order to significantly alter the system's optical properties. The large coupling strength achieved in the atomicensemble realizations of optomechanics induces optical bistability at a mean-intracavity photon level below one (Gupta et al., 2007; Ritter et al., 2009). This achievement is desirable for applications ranging from optical communication to quantum computation (Cirac et al., 1997; Imamoglu et al., 1997).
The occurrence of bistability in optomechanical systems can be understood from the corresponding semiclassical equations of motion for the oscillator displacement $X$ and the coherent intracavity field amplitude $\alpha$ derived from Hamiltonian Eq. (60)

$$
\begin{aligned}
\ddot{X}+\omega_{m} X & =-\omega_{m} G|\alpha|^{2} \\
\dot{\alpha} & =\left(i\left(\delta_{C}-G X\right)-\kappa\right) \alpha+\eta .
\end{aligned}
$$

In the bad cavity regime, $\kappa \gg \omega_{R}$, the atoms move on a timescale which is large compared to the lifetime $(2 \kappa)^{-1}$ of intracavity photons. Correspondingly, the cavity field adiabatically follows the atomic dynamics according to

$$
|\alpha|^{2}=\frac{\eta^{2}}{\kappa^{2}+\left(\delta_{C}-G X\right)^{2}} .
$$

Retardation effects resulting in dynamical back-action cooling or heating of the mechanical element are neglected in this approximation. Inserting this expression into the equation of motion for $X$, Eq. (61), yields $\ddot{X}=-\frac{d}{d X} V_{\mathrm{OM}}(X)$. The optomechanical potential given by

$$
V_{\mathrm{OM}}(X)=\frac{1}{2} \hbar \omega_{m} X^{2}-\frac{\hbar \eta^{2}}{\kappa} \arctan \left(\frac{\Delta(X)}{\kappa}\right),
$$

captures the combination of the harmonic confinement and the cavity dipole forces. Here, $\Delta(X)=\delta_{C}-G X$ denotes the detuning between the driving laser and the atoms-shifted cavity resonance.
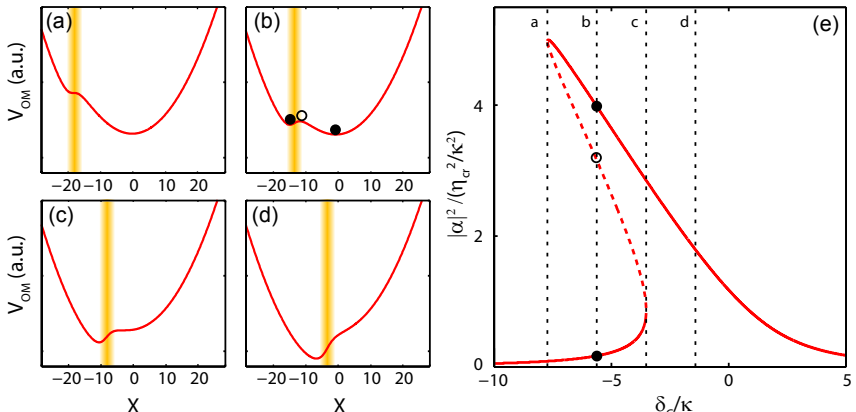

FIG. 25 (Color online) Optomechanical potential and bistability. (a-d) Optomechanical potential landscape $V_{\mathrm{OM}}(X)$ for different pump-cavity detunings $\delta_{C}$, indicated by the dashed lines in (e). The shaded regions show the resonance profile of the cavity. (e) Mean intracavity photon number $|\alpha|^{2}$ in steady state. Open and close circles correspond to the situation shown in (b). Parameters are $G=0.42 \kappa$ and $\eta=\sqrt{5} \eta_{\mathrm{cr}}$.

The optomechanical potential provides an intuitive picture to understand the steady-state as well as the dynamical behavior of the coupled system, see Fig. 25. Above a critical cavity pump strength $\eta_{\mathrm{cr}}$, determined by $\eta_{\mathrm{cr}}^{2}=\frac{8}{3 \sqrt{3}} \frac{\omega_{m} \kappa^{3}}{G^{2}}$, the optomechanical potential exhibits (within a certain detuning range) two local minima, which correspond to different intracavity light intensities as shown in the bistable resonance curve, Fig. 25e. 
Depending on the direction into which $\delta_{C}$ is adiabatically tuned, the system remains in either of the two local minimum of $V_{\mathrm{OM}}$, following the upper or lower bistable resonance branch. When reaching the critical detuning, where one of the local minima turns into a saddle point, the system starts to perform transient oscillations in the remaining potential minimum, which translate into a periodically modulated cavity light intensity. Due to damping of the collective atomic motion, the system finally relaxes to the steady-state in the remaining potential minimum.

Optical bistability induced by collective atomic motion was observed at low intracavity photon number both in the Berkeley group (Gupta et al., 2007) and the Zürich group (Ritter et al., 2009). The lower and upper bistability branches were observed in single experimental runs by slowly sweeping the frequency of the driving laser twice across resonance, first with increasing detuning and then with decreasing detuning, see Fig. 26. Upon increasing the probe strength, the cavity transmission profile becomes more and more asymmetric and exhibits hysteresis.

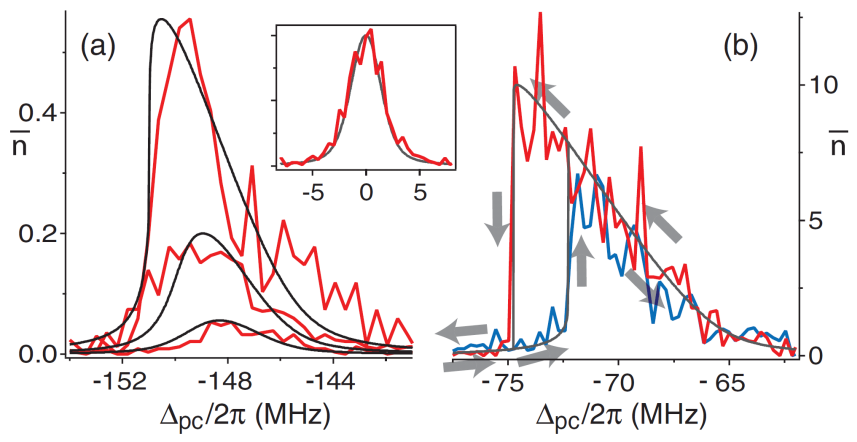

FIG. 26 (Color online) Dispersive optical bistability with collective atomic motion. (a) Observed cavity line shapes (red) for increasing cavity input power at low intracavity photon number $\bar{n}$ and model line shapes (black), based on the Voigtprofile of the bare cavity line (inset). $\Delta_{p c}$ denotes the detuning between the probe laser frequency and the empty cavity frequency. (b) Lower (blue) and upper (red) branches of optical bistability observed in single sweeps across resonance. From Gupta et al. (2007).

Dispersive optical bistability with collective atomic motion was also studied in the regime of quadratic optomechanical coupling (Purdy et al., 2010). Here, instead of displacing the center-of-mass motion of the mechanical element, the intracavity dipole forces increase or decrease the rms width of the compressible atomic ensemble, depending on whether the atoms are confined at a maximum or a minimum of the intracavity probe lattice potential. The corresponding change of the dispersive cavity shift leads again to bistable resonance curves as observed in the experiment (Purdy et al., 2010).

Dynamical optomechanical effects arise in small-

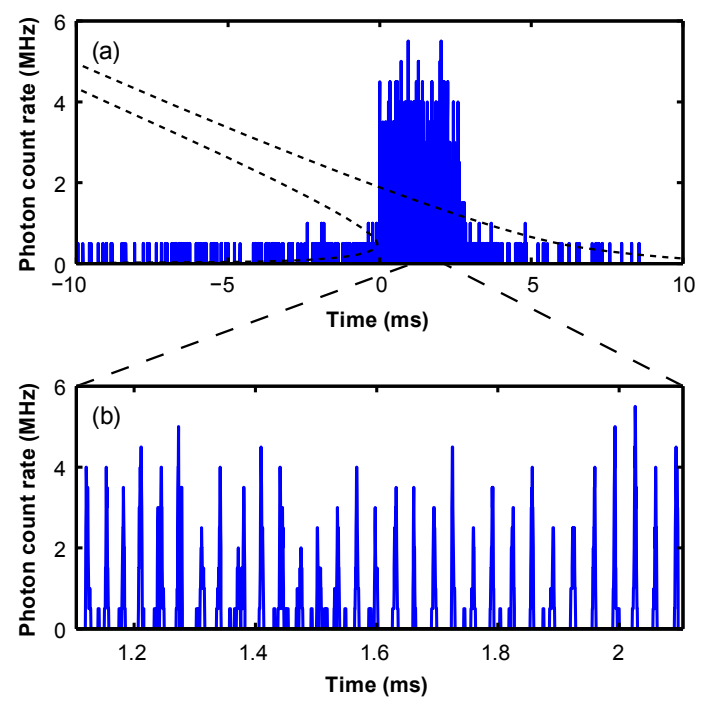

FIG. 27 Nonlinear dynamics of a driven BEC-cavity system. (a) Sweeping the driving laser frequency across the bistable resonance curve (indicated in dashed, scaled by a factor of 4) excites large-amplitude density oscillations in the condensate. (b) The magnified cavity transmission signal indicates how the density oscillations tune the cavity frequency periodically in an out of resonance with the driving laser. The mean intracavity photon number on resonance was 7.3 corresponding to a photon count rate of $5.8 \mathrm{MHz}$. Adapted from Brennecke et al. (2008).

amplitude oscillations of the system around steady state. As a result of the optomechanical interaction the frequency of such oscillations is shifted with respect to the bare oscillation frequency $\omega_{m}$, in the literature often referred to as the "optical spring effect". In the case of linear optomechanical coupling this can be inferred from a quadratic expansion around the steady state minima of the optomechanical potential $V_{\mathrm{OM}}$, Fig. 25. Experimentally, the optomechanical frequency shift for the collective atomic motion was observed and quantified in agreement with theory both in the linear and the quadratic coupling regime (Purdy et al., 2010). Highly nonlinear oscillations in the optomechanical potential with relatively large amplitude have been excited and observed in cavity transmission either by a sudden displacement of the optomechanical potential (Gupta et al., 2007) or by crossing the instability point of the bistable curves (Brennecke et al., 2008), see Fig. 27.

\section{Quantum-measurement back-action upon collective atomic motion}

The accuracy of any position measurement of a mechanical element is limited by quantum mechanics. Referred to as the standard quantum limit, this has been extensively studied in connection with the development of 
gravitational-wave detectors (Caves, 1980). In a generic optomechanical setup, which allows for high-precision measurements of the position of a mechanical element, the standard quantum limit arises from the balance between two noise terms: (i) detection shot noise, given by the random arrivals of photons on the detector and (ii) radiation-pressure induced displacement noise caused by the quantum fluctuations of the intracavity photon number. Whereas detection noise can be decreased by increasing the light power, this comes at the expense of increased radiation pressure force fluctuations. The optimal sensitivity is achieved if these two noise contribution are balanced. A direct experimental observation of the intracavity photon number fluctuations, requires large optomechanical coupling strengths between the intracavity field and the mechanical element in combination with the suppression of thermal or technical noise sources which perturb the mechanical motion.

The utilization of collective atomic motion of an ultracold gas strongly coupled to the field inside a FabryPérot resonator, allowed for the first observation of measurement-induced back-action upon a macroscopic mechanical element formed of $10^{5}$ atoms, caused by intracavity quantum force fluctuations (Murch et al., 2008). In the non-granular regime $\zeta=G / \kappa \ll 1$, the spectral density of intracavity photon number fluctuations (Marquardt et al., 2007; Nagy et al., 2009) agrees with that in an empty driven cavity, and reads

$$
S_{n n}(\omega)=\frac{2 \bar{n} \kappa}{\kappa^{2}+(\Delta(X)+\omega)^{2}} .
$$

Here, $\bar{n}=|\alpha|^{2}$ denotes the steady-state mean-intracavity photon number given in Eq. (62). These photon number fluctuations are transmitted into the momentum of the mechanical element via the optomechanical interaction, giving rise to a diffusion-like increase of the phonon number

$$
\frac{d}{d t}\left\langle c^{\dagger} c\right\rangle=\kappa^{2} \zeta^{2} S_{n n}\left(-\omega_{m}\right)
$$

as can be derived e.g. from an effective master equation for the mechanical oscillator (Nagy et al., 2009).

Murch et al. (2008) measured the corresponding heating rate of the atomic ensemble in a bolometric way by quantifying the evaporative atom loss, see Fig. 28. After preparing the mechanical oscillator close to its ground state, the cavity transmission of a weak probe beam at fixed frequency is monitored on a single-photon counting module. Continuous background atom loss tunes the atoms-shifted cavity frequency in resonance with the driving laser. The atom loss rate is deduced from the comparison between the recorded transmission curve and the empty-cavity resonance curve. The corresponding single-atom heating rate is found to exceed the free-space spontaneous heating rate, which was deduced by measuring the atom loss rate far from the cavity resonance, by
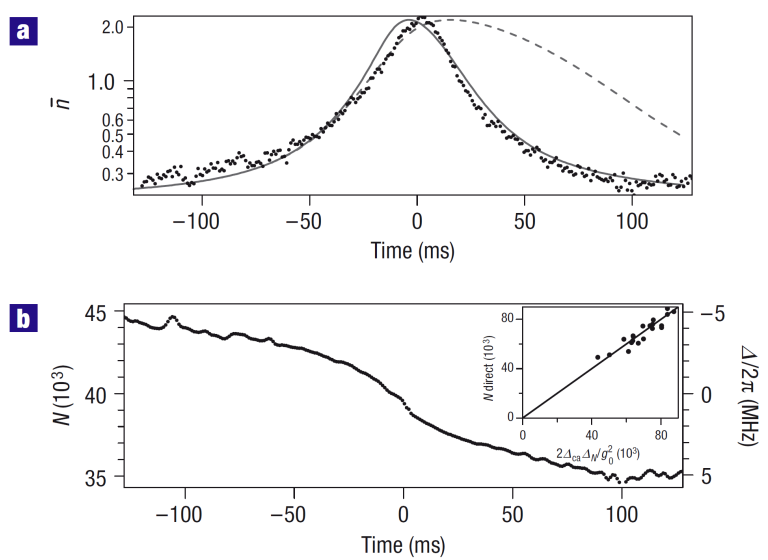

FIG. 28 Observing measurement-induced back-action upon the collective motion of an ultracold atomic ensemble. (a) Mean-intracavity photon number $\bar{n}$ (points), monitored as the system is brought across the cavity resonance due to evaporative atom loss. The expected photon number including (solid line) and excluding (dashed line) measurement back-action is shown. (b) Total atom number $N$ as a function of time as inferred from data shown in (a), using the empty cavity line shape and the linear relation between atom number and dispersive cavity shift (inset). From Murch et al. (2008).

a factor of 40, in agreement with the theoretical expectation. As cavity-mediated coherent amplification and damping of the mechanical oscillator is negligible in the experiment, the observation of back-action heating can be interpreted as a direct measurement of photon number fluctuations in a coherently driven cavity.

Another direct signature of quantum back-action of light upon collective atomic motion was obtained by monitoring the Stokes and anti-Stokes sidebands of the cavity transmission subsequent to the preparation of the collective motional degree of freedom close to its ground state (Brahms et al., 2012), see Fig. 29. The observed sideband asymmetry provides a direct measurement of the quantized collective motion and serves as a record of the energy exchanged between motion and the light in agreement with a continuous back-action limited quantum position measurement.

The disturbance of collective atomic motion via the intracavity quantum force fluctuations acts back again onto the intracavity light field. In particular, the resulting motional-induced modulation of the cavity field can interfere with the coherent or vacuum cavity input field giving rise to nonlinear optical parametric amplification and - for negligible technical or thermal fluctuations to ponderomotive squeezing (Fabre et al., 1994; Mancini and Tombesi, 1994). Only recently, these effects where observed for the first time in the Berkeley group utilizing the optomechanical coupling between collective atomic motion and an optical cavity field (Brooks et al., 2012). 


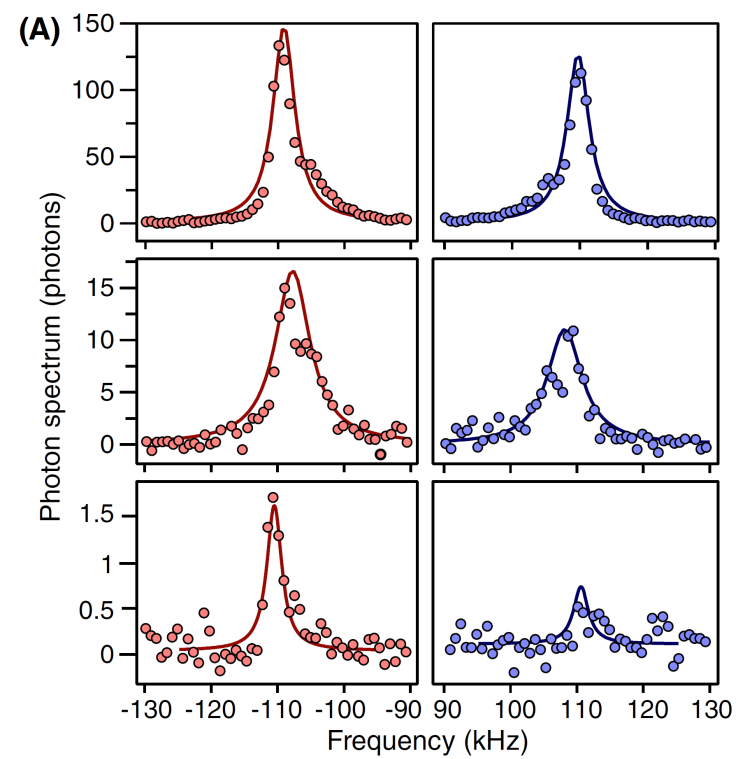

FIG. 29 Optical detection of quantization of collective atomic motion in the cavity output spectrum. Shown are measured Stokes sidebands (left panels) and anti-Stokes sidebands (right panels) for increasing intracavity photon number (bottom to top) together with the theoretical prediction (solid lines). The observed Stokes asymmetry provides a calibration-free measure for the mean occupation number of the mechanical oscillator, which was deduce for the lowest graph to be 0.49 . The mechanical oscillation frequency was $\omega_{m}=2 \pi \times 110 \mathrm{kHz}$. From Brahms et al. (2012).

\section{Cavity cooling in the resolved sideband regime}

For the small-volume cavities which were employed in the experiments performed by the Berkeley and Zurich group, the cavity decay rate $\kappa$ exceeds the mechanical oscillation frequency $\omega_{m}$ of the collective atomic degree of freedom by more than one order of magnitude. In this non-resolved sideband regime of cavity optomechanics cooling of the mechanical oscillator into its ground state utilizing cavity dissipation is not possible ${ }^{2}$. Rather, the minimal steady-state occupation number when driving the cavity field with a laser field which is red-detuned by $\sqrt{\omega_{m}^{2}+\kappa^{2}}$ from the cavity resonance is given by $\frac{\kappa}{2 \omega_{m}} \gg 1$ for weak optomechanical coupling strength (Marquardt et al., 2007).

Ground-state cooling becomes possible only in the resolved sideband regime where $\omega_{m} \gg \kappa$ (Kippenberg and Vahala, 2008). Here, the cavity is able to resolve the Stokes resp. anti-Stokes sidebands which correspond to adding resp. removing motional quanta from the mechanical degree of freedom. The large asymmetry between

\footnotetext{
${ }^{2}$ Indeed, in those experiments the preparation of the mechanical degree of freedom close to its ground state was achieved directly by evaporative cooling.
}

these processes which is achieved by driving the cavity near the anti-Stokes sideband results in a steady-state phonon occupation number of $\left(\frac{\kappa}{2 \omega_{m}}\right)^{2} \ll 1$ (Marquardt et al., 2007). Optomechanical cooling in the resolved sideband regime is equivalent to optical Raman sideband cooling of tightly confined atoms or ions.

Cavity cooling in an optomechanical-type BEC-cavity system which ranges in the good cavity regime, $\kappa<$ $\omega_{m}=4 \omega_{R}$, was demonstrated recently by Wolke et al. (2012). By driving the cavity field selectively close to the Stokes or anti-Stokes sidebands atoms where transferred via cavity-stimulating backward scattering from the macroscopically populated zero-momentum state into a superposition of momentum states $| \pm 2 \hbar k\rangle$ and back, see Fig. 30. This experiment paves the way towards the achievement of quantum degeneracy starting from a thermal gas without relying on evaporative cooling techniques.
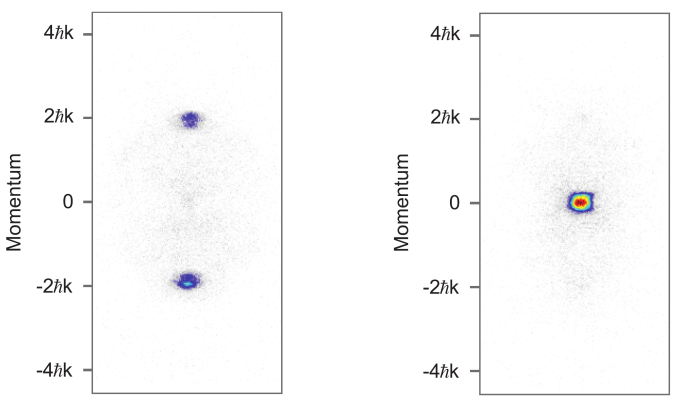

FIG. 30 Observation of sub-recoil cavity cooling with a ${ }^{87} \mathrm{Rb}$ BEC in a narrow-bandwidth Fabry-Pérot resonator. Shown are atomic momentum distributions after driving the cavity field with a far-detuned laser field at $803 \mathrm{~nm}$. First, a $400 \mu \mathrm{s}$ long pulse, blue-detuned from the cavity resonance, transfers atoms into the momentum states $| \pm 2 \hbar k\rangle$ (left), subsequently a $200 \mu$ s long red-detuned pulse transfers the atoms back into the zero-momentum state. Binary atomic collisions result in a substantial depletion of the $\pm 2 \hbar k$-momentum state populations visible as a diffusive halo. From Wolke et al. (2012).

\section{Non-equilibrium phase transitions}

Self-organization of a laser-driven atomic ensemble inside an optical resonator, as was considered for thermal atoms in Section III.B.1, was extended both theoretically and experimentally into the ultracold regime, where atomic motion becomes quantized. Correspondingly, the transition point to the self-organized phase is not determined anymore by thermal density fluctuations, rather by the competition between kinetic energy cost and potential energy gain associated with a spatial modulation of the atomic matter-wave in the cavity-induced lattice potential. In case of a weakly interacting BoseEinstein condensate, the reduced number of momentum 
states accompanying in the dynamics allows for a simplified description in terms of a collective spin degree of freedom, providing a direct link between self-organization and an open-system realization of the Dicke quantum phase transition.

\section{Self-organization of a Bose-Einstein condensate}

Self-organization of a dilute Bose-Einstein condensate (BEC), which is located in a single-mode optical cavity and illuminated transversally to the cavity axis by a far-detuned laser field, was studied theoretically by Nagy et al. (2008). In terms of a mean-field description, the steady-state of the system was obtained from the equations of motion for the coherent cavity field amplitude $\alpha$ and the atomic mean-field $\varphi(\mathbf{r})$, see Eq. (57b) and (57a), setting the on-axis pump strength $\eta$ to zero. For simplicity only atomic motion along the cavity axis was taken into account. The numerical solution for the steady-state order parameter $\Theta=\langle\varphi|\cos (k x)| \varphi\rangle$, obtained by numerically propagating the equations of motion into imaginary time, is shown in Fig. 31. Above a critical two-photon Rabi frequency $\eta_{\text {eff }}$, the order parameter takes a nonzero value indicating self-organization of the atoms in a $\lambda$-periodic density pattern. A stability analysis of the non-organized steady state, $\Theta=0$, yields the following

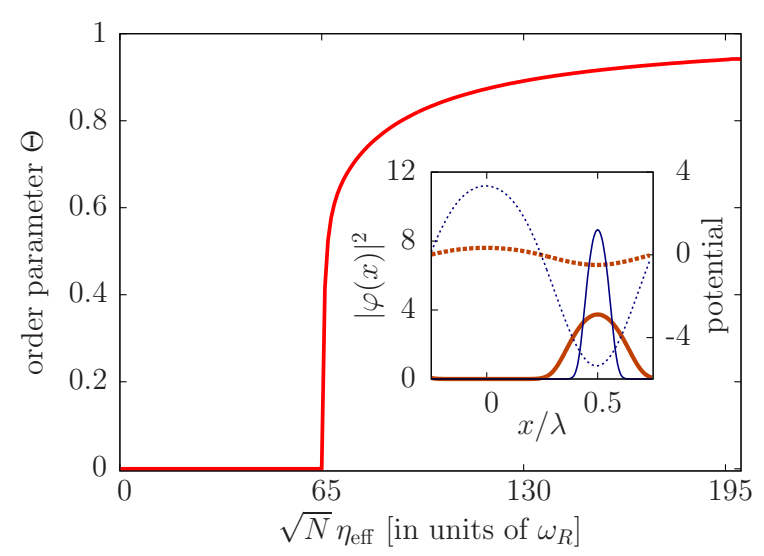

FIG. 31 Self-organization of a driven Bose-Einstein condensate in a standing-wave cavity. Plotted is the steadystate order parameter $\Theta$ as a function of the effective cavity pump strength $\eta_{\text {eff }}$, as obtained from a numerical solution of the mean-field equations. Parameters are $N U_{0}=-100 \omega_{R}$, $\Delta_{C}=-300 \omega_{R}, \kappa=200 \omega_{R}$ and $\mu_{0}=10 \hbar \omega_{R}$ in the homogeneous phase. According to Eq. (66) the homogeneous phase is stabilized in this parameter regime dominantly by collisional interaction energy. The inset shows the condensate wave functions (solid lines) for $\sqrt{N} \eta_{\text {eff }}=100 \omega_{R}$ (broader, brown solid) and $300 \omega_{R}$ (narrower, blue solid) and the corresponding optical dipole potentials (dashed lines). Adapted from Nagy et al. (2008).

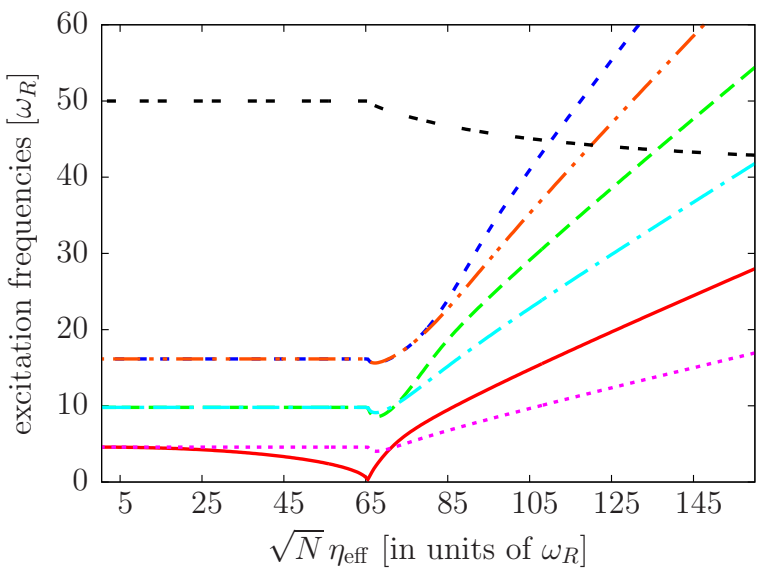

FIG. 32 Collective excitation spectrum of the transversally driven condensate-cavity system. Shown are the eigenfrequencies of the lowest six collective atomic-like and the first cavity-like (divided by 5) excited states as a function of the transverse pump amplitude. For vanishing pump amplitude the Bogoliubov spectrum $\Omega_{n}=\sqrt{n^{2} \omega_{R}\left(n^{2} \omega_{R}+2 \mu_{0}\right)}$ for a condensate in a 1D box potential of size $\lambda$ is retained. Self-organization is indicated by the softening of the lowest lying collective mode towards the critical pump amplitude $\sqrt{N} \eta_{\text {eff }, \mathrm{c}} \approx 65.5 \omega_{R}$. Parameters are the same as in Fig. 31. From Nagy et al. (2008).

analytic expression for the critical point

$$
\sqrt{N} \eta_{\text {eff,c }}=\sqrt{\frac{\left(\delta_{C}^{2}+\kappa^{2}\right)\left(\omega_{R}+2 \mu_{0} / \hbar\right)}{-2 \delta_{C}}}
$$

where $\mu_{0}$ denotes the chemical potential of the homogeneous condensate and $\delta_{C}$ the detuning of the pump laser from the dispersively shifted cavity resonance. In contrast to the thermal case Eq. (33), the critical transverse pump power scales in the zero-temperature limit with the recoil frequency (and the chemical potential), which reflects the fact that the homogeneous phase is stabilized by the kinetic energy (and atom-atom collisions).

A deeper understanding of the process of selforganization is gained from the collective excitation spectrum on top of the steady-state mean-field solution. This was calculated in Kónya et al. (2011) and Nagy et al. (2008) using a Bogoliubov-type approach based on the separation ansatz Eq. (56). The eigenvalues of the linearized equations for condensate and cavity fluctuations, Eq. (58), yield the energy spectrum of excitations (polaritons) shown in Fig. 32. For the considered case where the pump-cavity detuning $\delta_{C}$ is large compared to the recoil frequency $\omega_{R}$, the excitations separate into two classes, according to whether they are dominantly cavity-like or atom-like. The occurrence of self-organization is recognized in a characteristic softening of the atom-like excitation mode which matches the spatial interference pattern between cavity and transverse pump mode (Fig. 32, red solid line). 


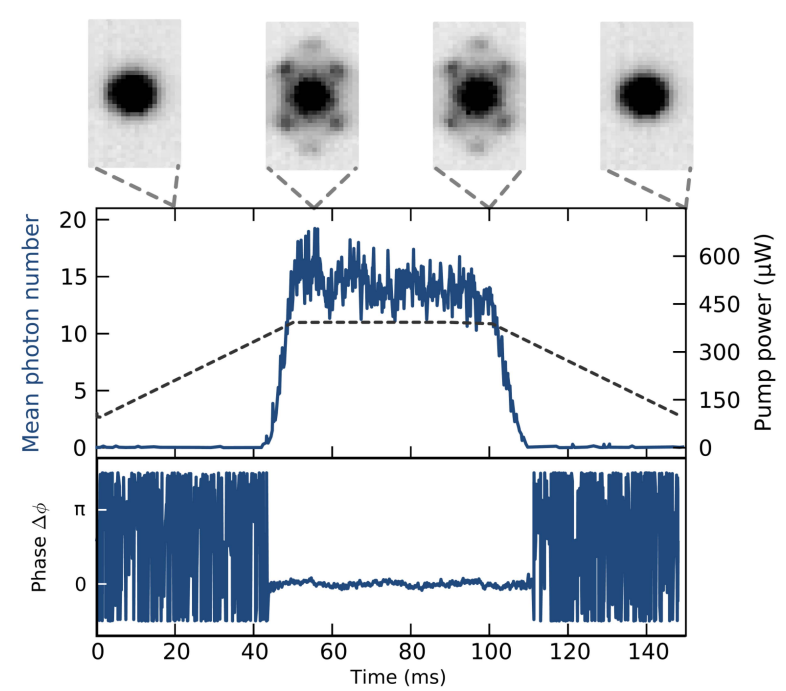

FIG. 33 Observation of self-organization with a Bose-Einstein condensate. Simultaneous time traces of the mean-intracavity photon number (middle panel) and the relative pump-cavity phase $\Delta \phi$ (lower panel) while ramping the transverse pump power twice across the critical point at $\approx 0.35 \mathrm{~mW}$. The absorption images (upper panel) show the atomic momentum distribution for the indicates times. The line of sight is perpendicular to the pump-cavity plane. Parameters are $\Delta_{C}=-2 \pi \times 20 \mathrm{MHz}, \kappa=2 \pi \times 1.3 \mathrm{MHz}$ and $N=10^{5}$. Adapted from Baumann et al. (2010, 2011).

Self-organization of a Bose-Einstein condensate was observed by the Zurich group (Baumann et al., 2010). A BEC of about $10^{5}$ atoms, harmonically confined inside a high-finesse optical Fabry-Pérot resonator, was illuminated by a far red-detuned standing-wave laser beam. By gradually increasing the power of the transverse laser beam, the transition to the self-organized phase was observed in a sharp raise of the intracavity light intensity accompanied by the build-up of macroscopic populations in the momentum states $\left(p_{x}, p_{z}\right)=( \pm \hbar k, \pm \hbar k)$, see Fig. 33 . Above the critical pump power, the relative phase $\Delta \phi$ between pump field and cavity field is observed to stay constant, which demonstrates that the system reached a steady state. By controlling the transverse pump power, the system can be transferred repeatedly from the normal in the self-organized phase and back (Fig. 33).

The process of symmetry breaking at the transition point was studied in Baumann et al. (2011). In repeated realizations of the self-organized phase, two possible values of the relative phase $\Delta \phi$ with a difference of $\pi$ were observed, according to self-organization into either the even $(u(x, z)>1)$ or odd $(u(x, z)<1)$ sites of the underlying mode interference mode profile $u(x, z)=\cos (k x) \cos (k z)$ (Fig. 34). The finite spatial extent of the atomic cloud results in a tiny imbalance between the even-odd populations in the non-organized phase. This effectively acts as a symmetry breaking field,

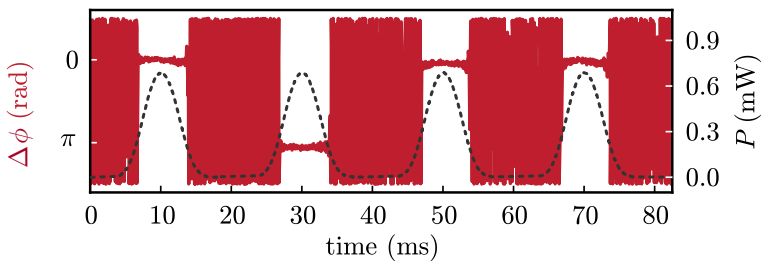

FIG. 34 Observation of symmetry breaking at the selforganization transition with a BEC. Shown is in red the relative pump-cavity phase $\phi$ monitored on a heterodyne detector while repeatedly entering the self-organized phase by tuning the transverse pump power $P$ (dashed). The system organizes into one out of two possible checkerboard patterns corresponding to the two observed phase values differing by $\pi$. From Baumann et al. (2010).

which favors the realization of one particular organized pattern, as was observed in the experiment. The influence of the symmetry breaking field could be overcome by increasing the speed at which the transition is crossed, in accordance with a simple model description based on the adiabaticity condition (Baumann et al., 2011).

In the limit where the cavity field adiabatically follows the atomic motion, the process of self-organization can also be understood as a result of the cavity-mediated atom-atom interactions, see III.A.1. On a microscopic level, these are induced by the virtual exchange of cavity photons between different laser-driven atoms, accompanied by the creation of atom pairs recoiling with momentum $\hbar k$ along the pump and cavity direction. The resulting $\lambda$-periodic density correlations in the atomic cloud energetically compete with the cost in kinetic energy, which gives rise to a characteristic roton-type softening in the dispersion relation of the condensate at momenta $( \pm \hbar k, \pm \hbar k)$, see also Fig. 32. Once the softened excitation energy reaches the ground state energy, the system self-organizes by macroscopically occupying those momentum states. Such mode softening was observed by the Zurich group (Mottl et al., 2012) using a variant of Bragg spectroscopy (Stenger et al., 1999) where the cavity field was probed with a weak laser pulse whose frequency was detuned by a variable amount from the transverse pump laser field. The observed excitation spectrum as a function of sign and modulus of the cavity-mediated atomatom interaction strength $V$ is depicted in Fig. 35. The vanishing of the excitation gap at the transition point towards the organized phase is accompanied by a diverging susceptibility of the system to $\lambda$-periodic density perturbations (Mottl et al., 2012). As was theoretically considered by Öztop et al. (2012), the softened excitation spectrum can also be probed parametrically via amplitude modulation of the transverse pump laser.

Conceptually, the self-organized BEC can be regarded as a supersolid (Gopalakrishnan et al., 2009; Leggett, 1970), similar to those proposed for two-component sys- 


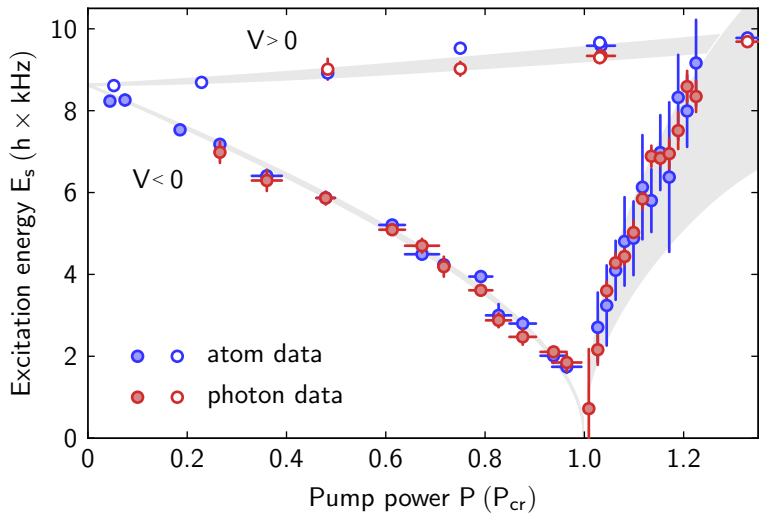

FIG. 35 (Color online) Observation of mode softening induced by cavity-mediated atom-atom interactions in a BoseEinstein condensate. Shown is the motional atomic excitation energy at momenta $( \pm \hbar k, \pm \hbar k)$ along the cavity and pump direction as a function of the transverse laser power $P$, which sets the modulus $|V|$ of the cavity-mediated atom-atom interaction. The sign of $V$ is determined by the sign of $\delta_{C}$. For negative interaction strength $V$, the system organizes at the critical pump power $P_{\text {cr }}$, while for positive interaction an increased excitation energy is observed in accordance with the absence of a phase transition. From Mottl et al. (2012).

tems (Büchler and Blatter, 2003). Non-trivial diagonal long-range order is induced by the cavity-mediated longrange interactions, which restricts the periodic density modulation to two possible checkerboard patterns, in contrast to traditional optical lattice experiments with laser fields propagating in free space. Simultaneously, the organized phase exhibits off-diagonal long-range order which is not destroyed while crossing the phase transition. Only when deeply entering the organized phase, tunneling between different sites of the optical checkerboard potential gets suppressed and phase coherence is lost (Vidal et al., 2010).

\section{Open-system realization of the Dicke quantum phase transition}

Self-organization of a laser-driven BEC in an optical resonator can be considered as an open-system realization of the Dicke quantum phase transition, where the quantized atomic motion acts as a macroscopic spin which strongly couples to the cavity field. The Dicke model goes back to the pioneering work of R. W. Dicke (Dicke, 1954) and describes the collective interaction between matter and the electromagnetic field. Consider $N$ two-level systems with transition frequency $\omega_{0}$, forming a collective spin variable $\mathbf{J}$, which couple identically to a single resonator mode at frequency $\omega$. This system can be described in terms of the Dicke Hamiltonian (also referred to as the Tavis-Cummings model (Tavis and Cum- mings, 1968))

$$
H_{\text {Dicke }} / \hbar=\omega a^{\dagger} a+\omega_{0} J_{z}+\frac{\lambda}{\sqrt{N}}\left(J_{+}+J_{-}\right)\left(a+a^{\dagger}\right)
$$

with the collective coupling strength denoted by $\lambda \propto$ $\sqrt{N}$. The ladder operators $J_{ \pm}=J_{x} \pm i J_{y}$ describe creation and annihilation of collective atomic excitations.

According to Dicke (1954), a collectively excited medium, which carries correlations among the different atomic dipoles, decays within a much shorter time into its ground state than a single atom. This phenomenon, termed superradiance (or superfluorescence), originates from spontaneous phase-locking of the different radiators resulting in a short radiation burst whose intensity is proportional to the number of atoms squared. Superradiant emission of laser-excited media has been studied extensively in the past (Gross and Haroche, 1982).

In contrast to this transient phenomenon, the Dicke Hamiltonian Eq. (67) was shown in 1973 to exhibit also a ground-state version of superradiance (Carmichael et al., 1973; Hepp and Lieb, 1973; Lambert et al., 2004; Wang and Hioe, 1973). When the collective coupling strength $\lambda$ reaches the critical value $\lambda_{\text {cr }}=\sqrt{\omega \omega_{0}} / 2$, the Dicke model undergoes a quantum phase transition from a normal into a superradiant phase, which is characterized by a macroscopic cavity field amplitude $\langle a\rangle$ and a macroscopic polarization $\left\langle J_{-}\right\rangle$of the atomic medium. Apart from its fragility upon the inclusion of the $A^{2}$ term originating from the minimal coupling Hamiltonian (Rzażewski et al., 1975), the experimental realization of the superradiant Dicke phase transition with direct dipole transitions was obscured in the past due to practical limitations in the available dipole coupling strengths.

The proposal by Dimer et al. (2007) circumvents these issues by considering a pair of stable atomic ground states which are coupled via two different Raman transitions involving a single ring cavity mode and external laser fields. This scheme realizes the Dicke model through an effective Hamiltonian in an open-system dynamics, including external driving and cavity loss, where the critical coupling strength can be reached for realistic experimental parameters.

The transversally driven BEC-cavity system is formally equivalent to this proposal upon replacing the electronic atomic states by a pair of motional atomic states, as was shown in Baumann et al. (2010) and Nagy et al. (2010). The two motional states are given by the flat condensate mode $\left|p_{x}, p_{z}\right\rangle=|0,0\rangle$ and the coherent superposition of the four momentum states $| \pm \hbar k, \pm \hbar k\rangle$, where $x$ and $z$ denote the cavity and pump direction, respectively. Coherent light scattering between the transverse pump beam and the cavity mode couples these two momentum states via two distinguishable Raman channels, resulting in a dipole-type interaction between cavity mode and the corresponding collective spin degree of freedom, see Eq. (67). The parameters $\left(\omega_{0}, \omega, \lambda\right)$ of 


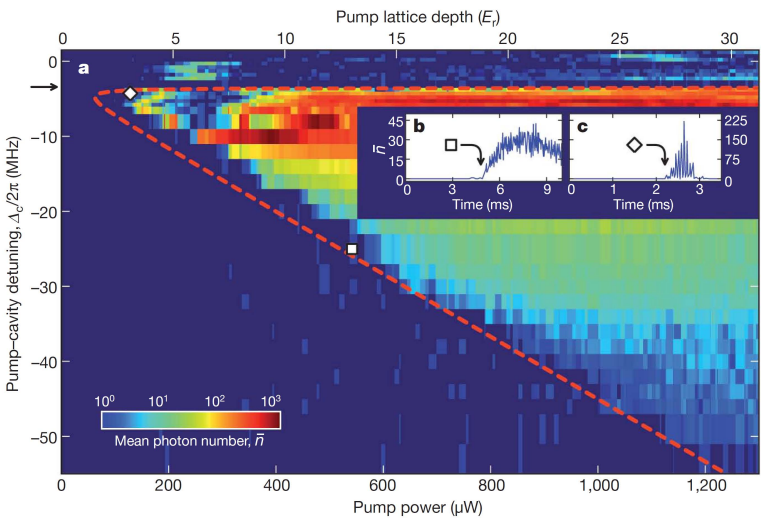

FIG. 36 (Color online) Dicke model phase diagram. (a) Displayed is in color the recorded mean intracavity photon number $\bar{n}$ as a function of the transverse pump power $P$ and the pump-cavity detuning $\Delta_{C}$. A sharp phase boundary is observed in agreement with a mean-field description (dashed line). The dispersively shifted cavity resonance for the nonorganized BEC is indicated by a horizontal arrow. (b,c) Time traces of $\bar{n}$ while gradually increasing the pump power to $1.3 \mathrm{~mW}$ for the indicated pump-cavity detuning. From Baumann et al. (2010).

the corresponding realization of the Dicke Hamiltonian are given by the energy difference $2 \omega_{R}$ between the two momentum modes (neglecting atom-atom collisions), the effective detuning $-\delta_{C}$ between the pump laser frequency and the dispersively shifted cavity mode frequency, and the collective two-photon Rabi frequency $\sqrt{N} \eta_{\text {eff }} / 2$ between pump laser and cavity mode, respectively. In the experiment (Baumann et al., 2010), $\delta_{C}$ exceeds the recoil frequency by three orders of magnitude, thus realizing the dispersive regime of the Dicke model. Higher-order momentum modes do not contribute in the phase transition dynamics itself and are populated only when deeply entering the self-organized phase (Kónya et al., 2011).

From the analogy to the Dicke model the following expression for the critical coupling strength is obtained upon including cavity decay (Dimer et al., 2007)

$$
\lambda_{\mathrm{cr}}=\sqrt{\frac{\left(\kappa^{2}+\omega^{2}\right) \omega_{0}}{4 \omega}} .
$$

In the absence of atom-atom collisions, this condition agrees with the result obtained from the stability analysis of the mean-field equations, Eq. (66). Experimentally (Baumann et al., 2010), the phase boundary was mapped out as a function of pump-cavity detuning $\Delta_{C}$ in agreement with the theoretical prediction, see Fig. 36.

It is instructive to contrast the Dicke quantum phase transition, realized with a BEC in a single-mode cavity, with the occurrence of free-space superradiant Rayleigh scattering off an elongated BEC which is driven by offresonant laser light (Inouye et al., 1999). In this experiment, a superradiant light pulse was emitted along the axial direction of the atomic cloud accompanied by the creation of recoiling matter-wave components, once the pump intensity exceeded a critical value. This dynamical effect is equivalent to Dicke superradiance of a collectively excited medium (Dicke, 1954), where matterwave amplification phase-locks the spontaneous emission events into the continuum of optical field modes. The minimal pump intensity required for superradiance to occur is determined by the balance between loss and gain processes. In contrast, light scattering off the BEC into a single cavity mode is a reversible process and the critical pump strength dominantly results from the finite pumpcavity detuning.

In the self-organized phase the detuning $\delta_{C}$ between pump laser and the dispersively shifted cavity resonance becomes a dynamic quantity. This effect is not taken into account by the description in terms of the Dicke model Eq. (67) which is a valid approximation as along as the maximum dispersive cavity shift $U_{0} N$ is small compared to the pump-cavity detuning $\left|\Delta_{C}\right|$. In the case where $U_{0} N$ exceeds $\left|\Delta_{C}\right|$ the system can exhibit dynamically frustrated behavior characterized by a periodic sign change of the effective pump detuning from the dispersively shifted cavity resonance, as was observed in Baumann et al. (2010), see Fig. 36c. Theoretically the influence of the additional nonlinear dispersive term $\sim U_{0} J_{z} a^{\dagger} a$ appearing in the Dicke model Eq. (67) upon the dynamics of the coupled BEC-cavity system was investigated in great detail by Bhaseen et al. (2012); Keeling et al. (2010b); and Liu et al. (2011). Employing a semiclassical description, Bhaseen et al. (2012) reveal a rich phase diagram including distinct superradiant fixed points, bistable and multistable coexistence phase and regimes of persistent oscillations, and explore the timescales for reaching these asymptotic states. It is emphasized that the behavior of the open system is controlled by the stable attractors, which do not necessarily coincide with the points of minimal free energy. As such, there is a crucial distinction between the $\kappa \rightarrow 0$ limit of the dynamical system and the equilibrium behavior at $\kappa=0$. Similar conclusion can be drawn in the quantum case, as discussed in the following.

The coupling of the cavity field to the electromagnetic field environment, causing cavity decay, amounts to a weak measurement of the coupled BEC-cavity system. The corresponding quantum back-action results in a diffusion-like depletion of the ground state of the Dicke Hamiltonian, even at zero temperature. The underlying physics is similar to that described in section IV.C.3, with the important difference that the system gets increasingly susceptible to quantum back-action when approaching the critical point.

The rate at which the ground state of the Dicke Hamiltonian initially gets depleted due to cavity decay was calculated in Nagy et al. (2010) based on the Langevin equation approach Eq. (58). In the dispersive regime 

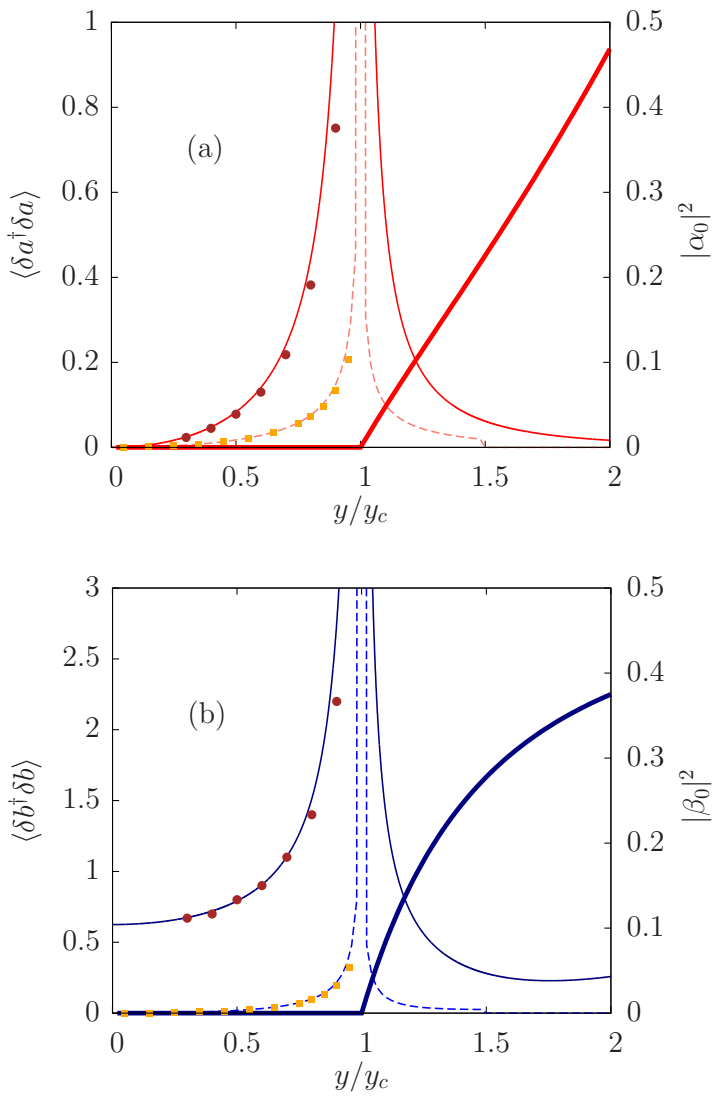

FIG. 37 (Color online) Criticality in the closed and opensystem Dicke phase transition. The mean values (thick, right axes) and the incoherent excitation numbers (thin, left axes) of the photon (a) and atomic (b) fields are plotted as a function of relative coupling strength $y / y_{\mathrm{cr}}=\lambda / \lambda_{\mathrm{cr}}$. The incoherent excitation numbers in steady-state for the open system (thin solid) diverge at the critical point with exponent -1 in contrast to the ground-state number of excitations for the closed system (thin solid), which diverges with the mean-field exponent $-1 / 2$. From Nagy et al. (2011).

$\left|\delta_{C}\right| \gg \omega_{R}$, the ground state depletion happens mostly in the atomic space and the corresponding diffusion rate can be approximated below threshold by the expression $\omega_{R} \kappa /\left|\delta_{C}\right|\left(\lambda / \lambda_{\text {cr }}\right)^{2}$. Per atom, this corresponds for $\left|\delta_{C}\right| \gg \kappa$ to a heating rate of $\kappa \frac{\eta_{\text {eff }}^{2}}{\delta_{C}^{2}}$. Note, the formal equivalence of this result with the spontaneous heating rate in a far-detuned dipole trap. Importantly, the use of a large detuning $\left|\delta_{C}\right|$ removes the time limitation imposed by measurement-induced back-action.

Measurement-induced back-action drives the BECcavity system into a steady state which is a dynamical equilibrium between diffusion and damping. It is very interesting that this limiting state is not the same as the equilibrium state of the system, i.e., for $\kappa=0$ the ground state at $T=0$. Namely, the order of the two limiting procedures, $t \rightarrow \infty$ and $\kappa \rightarrow 0$ cannot be interchanged. The steady-state $(t \rightarrow \infty$ limit) occupation of the cavity field and the excited momentum state were calculated in Nagy et al. (2011). Flux and second-order time correlations of the cavity output signal were investigated theoretically in Öztop et al. (2012). The mean-field obtained in the thermodynamic limit is a smooth function of $\kappa$ and the steady-state solution tends to that of the ground state of Eq. (67) for $\kappa \rightarrow 0$. By contrast, the comparison of the quantum fluctuations present in the ground state of the Dicke model and in the steadystate of the damped-driven system exhibit a significant difference. In the ground-state of Eq. (67) the secondorder correlation functions diverge towards the critical point with the exponent $-1 / 2$, indicating a mean-fieldtype transition, whereas in the non-equilibrium case the quantum fluctuations exhibit a divergence with exponent -1 (see Fig. 37). At the same time, the singularity of the ground-state entanglement (Lambert et al., 2004) between cavity and atomic subsystem is regularized at the critical point by the quantum noise associated with cavity decay. The non-vanishing entanglement, however, shows that the quantum character of the Dicke quantum phase transition (self-organization at zero temperature) is not fully destroyed in case of an open-system dynamics, and it cannot be exactly mapped to a thermal noise-driven phase transition.

\section{Phases in highly degenerate cavities}

Self-organization of polarizable particles into periodic structures induced and stabilized by the intracavity light field resembles the process of crystallization. In a cavity with only a single standing-wave mode tuned into resonance with the pump field, only the amplitude of the cavity field is a dynamical quantity. The formation of a periodic crystal from a spatially homogeneous distribution breaks the discrete symmetry corresponding to the even and odd antinodes of the standing-wave mode profile. Already in the two-mode setting of a ring cavity sustaining two degenerate counter-propagating modes, the selforganization is accompanied by spontaneous breaking of a continuous translational symmetry (Nagy et al., 2006b; Niedenzu et al., 2010), which induces rigidity against lattice deformations. In the case of highly degenerate multimode cavities the field has much more freedom to adjust locally to the particle distribution.

The general structure of the resulting complex phase diagram was studied in Gopalakrishnan et al. (2009) and Ritsch (2009). In general such setups allow to realize conceptually novel systems and to explore and discover properties of crystalline and liquid-crystalline ordering, including intrinsic effects as dislocations, the growth and arrangement of crystal grain boundaries (see Fig. 38), and the nature of the phonon spectrum. Multimode cavities also offer a natural connection to models developed in the field of neural networks as the Hopfield model or sim- 
ilar spin models with infinite range statistical couplings. First ideas about this relation have been raised very recently in Gopalakrishnan et al. (2011a,b); and Strack and Sachdev (2011). Extensions to fermionic atoms in multimode cavities have been considered in Müller et al. (2012).

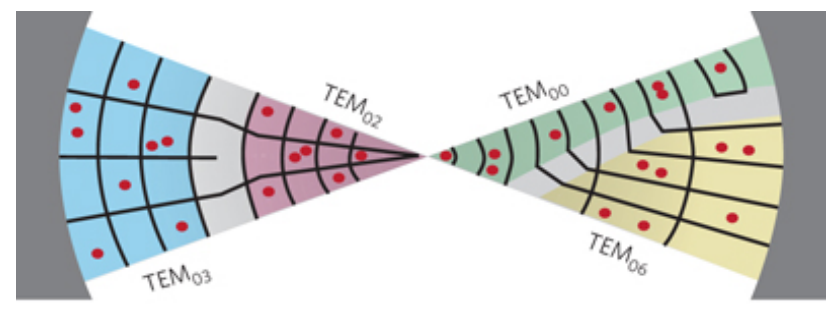

FIG. 38 (Color online) Self-ordered states in a concentric multimode cavity forming two-dimensional patterns. The diagram shows a regime near threshold, with domains locally populating distinct $\mathrm{TEM}_{x y}$ cavity modes in the equatorial plane. Domains can be punctuated by dislocations (shown in the left half of the figure), but might also show textural variation in space (right half of the figure). The black lines represent nodes of the cavity field, which separate 'even' and 'odd' antinodes. As the atoms are Bose-condensed, the atomic population per site is not fixed. From Gopalakrishnan et al. (2009).

Gopalakrishnan and coworkers generalized and adapted a field-theoretical framework, also successfully used in solid-state physics, to describe many-body systems coupled to a multitude of degenerate modes of a high-finesse cavity (Gopalakrishnan et al., 2010; Keeling et al., 2010a). For a quasi-two-dimensional cloud of atoms confined in the equatorial plane of a concentric optical cavity, the transition from the homogeneous distribution into a spatially modulated one is of the Brazovskii type (Brazovskii, 1975), which describes the phase transitions from isotropic to striped structures in liquid crystals. The description is based on an effective equilibrium theory which is valid when the effective cavity loss rate $\kappa \eta_{\text {eff }}^{2} / \Delta_{C}^{2}$ is smaller than the recoil frequency $\omega_{R}^{-1}$, see IV.D.2. Here, the dispersive cavity shift was assumed to be much smaller than the pump-cavity detuning $\Delta_{C}$. Unlike the Landau theory of crystallization, here the free energy of the system does not have a cubic term that breaks the symmetry at the phase transition. The transition persists at zero temperature, hence it realizes a quantum phase transition of an unusual university class. The non-equilibrium extension of this theory, which includes the effect of photon leakage out of the cavity as a perturbation, leads to the conclusion that the photon loss corresponds to an effective temperature and quantum correlations are washed out by decoherence on timescales longer than the cavity decay time. Note that the Bogoliubovtype mean-field model description of the open-system Dicke-model in a single mode cavity also predicts the depletion of the ground state (Nagy et al., 2010) due to measurement-induced back-action (Murch et al., 2008), or, in other words, due to the diffusion induced by fluctuations accompanying cavity photon loss (Nagy et al., 2009). However, unlike the Brazovskii transition, the Dicke-model system is driven into a steady-state, significantly different from the ground state, which has a critical point (Nagy et al., 2011).

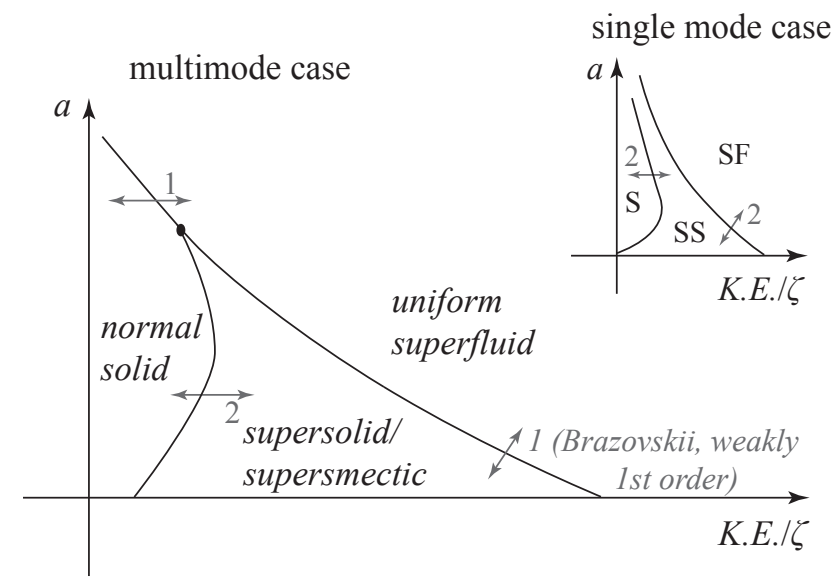

FIG. 39 Schematic zero-temperature phase diagram for a BEC in a concentric cavity. The control parameters are the atomic scattering length $a$ and the inverse effective atomcavity coupling $\zeta^{-1}$ with $\zeta=\eta_{\text {eff }}^{2} / \Delta_{C}$. For weak, repulsive interactions and increasing atom-cavity coupling, the superfluid first undergoes self-organization via the Brazovskii transition, thus forming a supersolid. If the transverse laser intensity is increased further, the supersolid undergoes a transition into a normal solid (i.e., a Mott insulator). For strong, repulsive interactions, the uniform BEC can lose phase coherence concurrently with a first-order self-organization transition. This situation is to be contrasted with that for the case of a singlemode cavity (inset), in which there should always be a supersolid (SS) region separating the uniform fluid (SF) and normal solid (S) regions. First- and second-order transitions are marked by 1 and 2, respectively. From Gopalakrishnan et al. (2010).

Similarly to the case of the single-mode experiment performed in the Zurich group (Baumann et al., 2010), the emergent crystalline state in a transversally driven multimode cavity can be considered as a supersolid phase where crystalline order and off-diagonal long-range order (long-range phase coherence) coexist. The phase diagram, schematically shown in Fig. 39 for a multimode cavity is strikingly different from the single-mode case in that a region with direct uniform superfluid-to-normal phase transition occurs, whereas in the single-mode cavity there is always a supersolid state between the uniform and the normal solid phases (Vidal et al., 2010). It is also observed that, for a strongly layered three-dimensional structure, the inter-layer frustration precludes global ordering and the system breaks up into inhomogeneous domains. 


\section{E. Extended Hubbard-type models for ultracold atoms in cavities}

The theoretical description of the quantum many-body dynamics of ultracold atoms confined in optical lattices and strongly interacting with a quantized cavity field can be based on a sophisticated extension of the BoseHubbard (BH) model (Fisher et al., 1989). In static optical lattices, the $\mathrm{BH}$ model properly accounts for the quantum statistical properties of bosonic atoms at the lattice sites, as well as the inter-particle quantum correlations (Bloch et al., 2008). The basic assumption, valid in the limit of very low temperature, is that the dynamics can be restricted to the lowest (or lowest few) Bloch bands of the periodic optical potential. Correspondingly, the many-body wavefunction can be expressed in terms of Wannier functions localized at individual lattice sites.

However, if the optical lattice potential is sustained by the mode of a high-finesse cavity, thus becoming a dynamical degree of freedom, it gets a highly nonlinear problem to determine the Wannier functions themselves and thereby the ground state of the many-body system. For a laser-driven cavity, e.g., the atoms dispersively shift the cavity resonance, thus affecting the intracavity field amplitude which itself determines the lattice depth. Hence, the optical lattice potential and the state of the atoms have to be evaluated in a self-consistent way (Larson et al., 2008a; Maschler et al., 2008; Maschler and Ritsch, 2005; Vidal et al., 2010), as will be presented below. We focus on the most studied particular case of spinless bosons, and mention only that the cases of fermions and spin particles are expected to lead to interesting novel effects (Larson et al., 2008c; Sun et al., 2011).

\section{Bose-Hubbard model with cavity-mediated atom-atom interactions}

Consider an ensemble of $N$ bosonic particles subject to an optical lattice potential which is generated by the field of an optical resonator and possibly by an additional, far off-resonant standing-wave laser field. The latter is represented by the external potential term $V_{\mathrm{cl}}(\mathbf{r})$ in the single-atom Hamiltonian Eq. (51). Restricting the motional dynamics to the lowest energy band (lowest vibrational state), we expand the atomic field operator

$$
\Psi(\mathbf{r})=\sum_{i=1}^{M} b_{i} w\left(\mathbf{r}-\mathbf{r}_{i}\right)
$$

in the Wannier basis of atomic states localized at sites $i=1 \ldots M$, where $b_{i}$ denotes the associated annihilation operator. Upon inserting this expansion into Eqs. (49), one obtains

$$
\begin{gathered}
H=\sum_{m}\left(-\hbar \Delta_{C, m} a_{m}^{\dagger} a_{m}+i \hbar \eta_{m}\left(a_{m}^{\dagger}-a_{m}\right)\right) \\
+\sum_{i, j=1}^{M}\left(E_{i, j}+V_{\mathrm{cl}} J_{i, j}^{\mathrm{cl}}\right) b_{i}^{\dagger} b_{j} \\
+\frac{\hbar}{\Delta_{A}} \sum_{l, m} g_{l} g_{m} a_{l}^{\dagger} a_{m}\left(\sum_{i, j=1}^{M} J_{i, j}^{l m} b_{i}^{\dagger} b_{j}\right) \\
+\frac{U}{2} \sum_{i=1}^{M} b_{i}^{\dagger} b_{i}\left(b_{i}^{\dagger} b_{i}-1\right),
\end{gathered}
$$

where several cavity modes with mode functions $f_{m}(\mathbf{r})$ and corresponding photon annihilation operators $a_{m}$ are considered. The coefficients $E_{i, j}$ and $J_{i j}^{\text {cl }}$ are defined as in the standard $\mathrm{BH}$ Hamiltonian

$$
\begin{aligned}
& E_{i, j}=\int d^{3} \mathbf{r} w\left(\mathbf{r}-\mathbf{r}_{i}\right)\left(-\frac{\hbar^{2} \nabla^{2}}{2 m}\right) w\left(\mathbf{r}-\mathbf{r}_{j}\right) \\
& J_{i, j}^{\mathrm{cl}}=\int d^{3} \mathbf{r} w\left(\mathbf{r}-\mathbf{r}_{i}\right) f_{\mathrm{cl}}(\mathbf{r}) w\left(\mathbf{r}-\mathbf{r}_{j}\right)
\end{aligned}
$$

where we separated the characteristic magnitude $V_{\mathrm{cl}}$ of the classical trapping potential (difference between maximum and minimum) from its spatial form $f_{\mathrm{cl}}(\mathbf{r})$. The last term of Eq. (70) describes the on-site interaction with $U=\left(4 \pi a_{s} \hbar^{2} / m\right) \int d^{3} \mathbf{r}|w(\mathbf{r})|^{4}$. Of primary interest are the extra couplings generated by the cavity modes with matrix elements

$$
J_{i, j}^{l m}=\int d^{3} \mathbf{r} w\left(\mathbf{r}-\mathbf{r}_{i}\right) f_{l}^{*}(\mathbf{r}) f_{m}(\mathbf{r}) w\left(\mathbf{r}-\mathbf{r}_{j}\right) .
$$

From the cavity field point of view, the diagonal elements, $l=m$, correspond to the atomic state-dependent dispersive shifts of the cavity mode frequency $\omega_{C, m}$, whereas the off-diagonal elements, $l \neq m$, describe photon scattering between different cavity modes. The Wannier functions $w\left(\mathbf{r}-\mathbf{r}_{i}\right)$ appearing in these integrals, in principle depend on the dynamic potential terms generated by the cavity field. This renders the problem highly non-trivial.

In the most general case, the Wannier functions have to be calculated for each photon number state to define a corresponding manifold of parameters in the $\mathrm{BH}$ model, Eq. (70). In other words, the couplings $J_{i, j}^{l m}$ are replaced by operators which can be easily expressed in a Fock basis. Such a brute force approach is necessary if the effect of the cavity field on the trapping potential is significantly different for adjacent Fock states (Horak and Ritsch, 2001b). Typically, numerical simulations have to be performed to study, e.g., microscopic processes underlying many-body effects that are understood in the meanfield limit (Maschler et al., 2007; Niedenzu et al., 2010; Vukics et al., 2007). Obviously, this approach is limited to small system sizes of a few particles moving in a few 


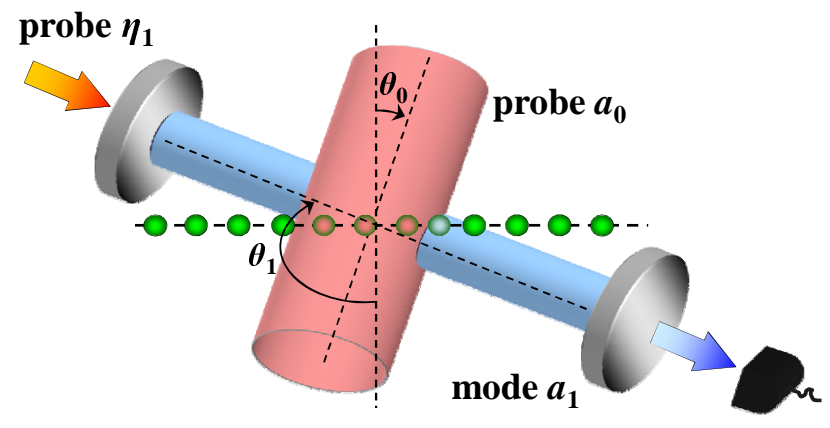

FIG. 40 (Color online) Scheme for quantum non-demolition measurement of atomic many-body states in an optical lattice. $N$ atoms are trapped in a one-dimensional lattice potential (green, $M$ lattice sites) which partially overlaps with a cavity mode $a_{1}$ (blue) and a transverse probe mode $a_{0}$ (red). The number of illuminated lattice sites is denoted by $K$. Depending on their many-body state the atoms act as a quantum refractive index whose statistical distribution with respect to the probe and cavity modes can be mapped out via transmission or diffraction spectroscopy as a function of the probecavity detuning or the angles $\Theta_{0}$ and $\Theta_{1}$.

cavity modes. Most of the works, however, used approximations to treat the cavity-generated optical potential in which the localized Wannier functions are defined in a self-consistent manner.

\section{Cavity-enhanced light scattering for quantum measurement and preparation}

Before addressing the problem of dynamical cavityinduced potentials within the framework of the $\mathrm{BH}$ model, we note that a lot of applications have been developed based on the coupling of quantized cavity field modes to trapped, ultracold atomic systems in a simple scattering regime, as was exhaustively reviewed by Mekhov and Ritsch (2012b). In the scattering scenario, the external lattice potential $V_{\mathrm{cl}}(\mathbf{r})$ is taken strong enough to define solely the localized Wannier functions, and their modification due to the cavity light forces is negligible. The quantized cavity field modes are a perturbative probe which can yield a mapping between quantum properties of atomic many-body states and light observables. This system gives, for example, a means to determine the quantum state of ultracold atoms by light scattering (Miyake et al., 2011).

A typical quantum measurement scheme involving a single cavity mode is depicted in Fig. 40. It was shown that various quantum states of ultracold bosons trapped in the lowest band of an optical lattice and having equal mean densities can be distinguished (Chen et al., 2007; Mekhov et al., 2007a). As a characteristic example, the very different transmission spectra of the Mott insulator (MI) and the superfluid (SF) states are exhibited in Fig. 41. In contrast to standard techniques this measure-

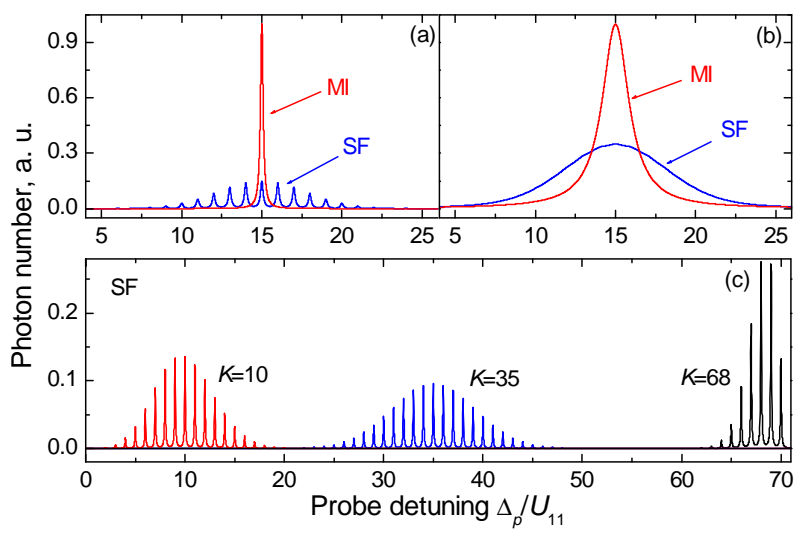

FIG. 41 (Color online) Cavity transmission spectra showing the atom number distribution of an ultracold gas in the intracavity part of an optical lattice, see Fig. 40. Shown are transmission profiles as a function of the probe-cavity detuning $\Delta_{p}$ of a MI (red) and a SF (blue) state for (a) a good cavity with $\kappa=0.1 U_{11}$ and (b) a bad cavity with $\kappa=U_{11}$, where $U_{11}=g_{1}^{2} / \Delta_{A}$. In (b) the satellites are not resolved but the spectra for SF and MI states are still different. Parameters are $N=M=30$ and $K=15$. (c) Spectra for a SF state with $N=M=70$ and different number of illuminated lattice sites K. From (Mekhov et al., 2007c).

ment is non-destructive, limited only by quantum measurement back-action. Depending on the chosen geometry, light scattering is sensitive to the global and local atom number fluctuations (Bhattacherjee et al., 2010; Chen and Meystre, 2009; Mekhov et al., 2007c), or to long-range correlations between two or more lattice sites (e.g. four-point correlations) (Bux et al., 2011; Mekhov et al., 2007b).

The back-action of repeated quantum non-demolition measurements drives the atomic many-body state towards specific states (Mekhov and Ritsch, 2009). This is in full analogy to microwave cavity QED experiments (Brune et al., 1990; Guerlin et al., 2007) where, complementarily, the field state is driven into nonclassical Fock states by measuring the state of a train of atoms crossing the cavity.

\section{Self-consistent Bose-Hubbard models in cavity mean-field approximation}

Genuine cavity-induced dynamical effects appear when the excited cavity modes significantly modify the trapping with respect to the external potential $V_{\mathrm{cl}}(\mathbf{r})$. Even without noticeably reshaping the Wannier-functions at the trapping sites, the perturbative light probe can modify the tunneling rates as was shown for free-space Bragg scattering by Rist et al. (2010). In a cavity, local changes of the atomic distribution influence the whole cavitysustained optical lattice potential. Thereby a new type of long-range interaction between the particles appears and 
gives rise to resonant nonlocal co-tunneling or momentum space pairing (Mekhov et al., 2007b), effects which go far beyond the standard Bose-Hubbard model.

When the Wannier functions themselves are dynamically influenced by the cavity, a self-consistent meanfield approach, similar to the one in Sec. IV.B, has been broadly adopted to describe the nonlinear dynamics of trapped, ultracold atoms in a cavity (Chen et al., 2009; Larson et al., 2008b; Larson and Lewenstein, 2009; Maschler et al., 2008; Maschler and Ritsch, 2005; Nimmrichter et al., 2010; Vidal et al., 2010). Splitting the cavity field amplitude $a=\langle a\rangle+\delta a$ into its mean value and fluctuations, the main assumption is that only the highly excited mean field can modify the trapping potential, whereas the fluctuations amount to a perturbative probe. Since the cavity mean-field amplitude $\langle a\rangle$ depends on the momentary atomic quantum state, so does the depth and shape of the on-site potential. Therefore, the Wannier functions in Eq. (69) and hence the coefficients Eq. (71) in the Hubbard-type Hamiltonian Eq. (70), have to be determined self-consistently in conjunction with the proper cavity mean field $\langle a\rangle$. It is noteworthy that, often, the self-consistent calculation does not lead to a unique solution.

a. Phases in dynamical optical lattices Consider a linear cavity with only a single mode being driven and overlapping with a static optical lattice potential $V_{\mathrm{cl}}(\mathbf{r})$. Assuming only nearest-neighbor hopping to be relevant we keep adjacent $\langle i, j\rangle$ pairs from the double sum over indices $i$ and $j$ in Eq. (70). The corresponding many-body Hamiltonian is given by

$$
\begin{array}{r}
H=E_{0} \hat{N}+E \hat{B}+\left(\hbar U_{0} a^{\dagger} a+V_{\mathrm{cl}}\right)\left(J_{0} \hat{N}+J \hat{B}\right) \\
-\hbar \Delta_{C} a^{\dagger} a-i \hbar \eta\left(a-a^{\dagger}\right)+\frac{U}{2} \hat{C} .
\end{array}
$$

The relevant atomic degrees of freedom are the total atom number $\hat{N}$ and the collective nearest-neighbor coherence $\hat{B}$ defined as

$$
\hat{N}=\sum_{i=1}^{M} b_{i}^{\dagger} b_{i}, \quad \hat{B}=\sum_{i=1}^{M} b_{i}^{\dagger} b_{i+1}+b_{i+1}^{\dagger} b_{i},
$$

respectively, and the operator $\hat{C}=\sum_{i} b_{i}^{\dagger} b_{i}\left(b_{i}^{\dagger} b_{i}-1\right)$ for the two-body on-site interaction. The coefficients $E_{0}$, $E, J_{0}$ and $J$ derive from Eq. (71) contracted to a single cavity mode and assuming uniform coupling along the lattice.

To exhibit the underlying physics one may neglect the photon number dependence of the Wannier functions and adiabatically eliminate the cavity field via the Heisenberg equation of motion

$$
\dot{a}=\left\{i\left[\Delta_{C}-U_{0}\left(J_{0} \hat{N}+J \hat{B}\right)\right]-\kappa\right\} a+\eta .
$$
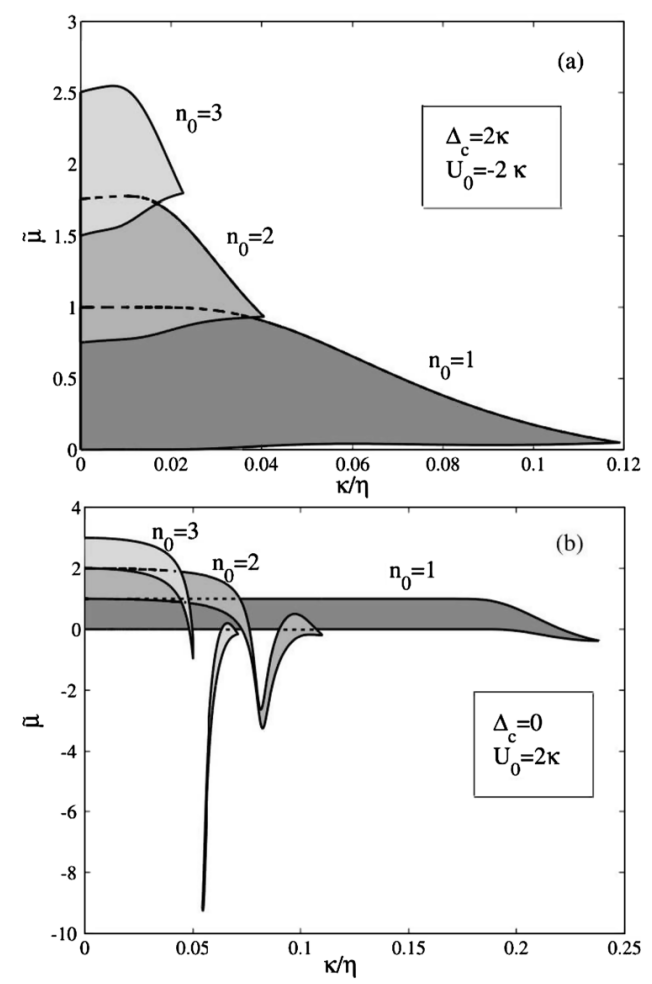

FIG. 42 Phase diagram with overlapping Mott insulator states. Boundaries of different Mott lobes (shaded regions) as a function of the rescaled chemical potential $\tilde{\mu}$ and the inverse of the pump strength $\eta$ (in units of $\kappa$ ) in the 1D cavity lattice potential of $K=50$ sites. Parameters are (a) $\left(\Delta_{C}, U_{0}\right)=(2 \kappa,-2 \kappa)$ and $(\mathrm{b})\left(\Delta_{C}, U_{0}\right)=(0,2 \kappa)$. The Mott lobes are labeled by the number of atoms per site $n_{0}$. The dashed lines show the boundaries of zones which are hidden. Outside the shaded parameter regions, the state of the system is superfluid in most cases. From (Larson et al., 2008a).

This is a good approximation as long as the cavity field decays fast compared to the timescale of atomic motion. Since tunneling in deep lattice potentials is slow compared to the recoil frequency, this applies widely in experimental setups.

An effective atomic Bose-Hubbard model, formally identical to the usual one but with coefficients $J_{0}$ and $J$ depending on the many-body state, has been considered systematically in the thermodynamic limit. By evaluating the stability of the Mott insulator states, the phase diagram has been constructed. As shown in Fig. 42, the model predicts the existence of competing Mott insulator states (Larson et al., 2008a,b). The overlapping Mott lobes indicate the possibility of bistability in this laserdriven, nonlinear system, see IV.C.2. The state of the system can be controlled by fine tuning the pump parameters near the shifted cavity resonance. For certain parameters a state with two atoms per site can lead to a much higher photon number and thus deeper optical potential, so that its energy falls below that of the state with unity filling. 
In order to gain insight into the nature of atom-atom coupling via the cavity field, a simple effective Hamiltonian can be constructed. The adiabatic field amplitude can be expanded to second order in the small tunneling matrix element $J$

$$
a \approx \frac{\eta}{\kappa-i \delta_{C}}\left[\mathbf{1}-i \frac{U_{0} J}{\kappa-i \delta_{C}} \hat{B}-\frac{\left(U_{0} J\right)^{2}}{\left(\kappa-i \delta_{C}\right)^{2}} \hat{B}^{2}\right],
$$

where the effective detuning $\delta_{C}=\Delta_{C}-U_{0} J_{0} N$ was introduced, and the atom number was set to $N$. Inserting this solution back into the Hamiltonian Eq. (72) and the Liouville operator Eq. (5a), which accounts for cavity damping, leads to an effective adiabatic model. It comprises the nonlinear Hamiltonian

$$
\begin{aligned}
H_{\mathrm{ad}} & =\left(E+J V_{\mathrm{cl}}\right) \hat{B}+\frac{U}{2} \hat{C} \\
+ & \frac{\hbar U_{0} J \eta^{2}}{\kappa^{2}+\delta_{C}{ }^{2}}\left(\hat{B}+\frac{U_{0} J \delta_{C}}{\kappa^{2}+\delta_{C}{ }^{2}} \frac{\kappa^{2}-3 \delta_{C}{ }^{2}}{\kappa^{2}+\delta_{C}{ }^{2}} \hat{B}^{2}\right),
\end{aligned}
$$

and Liouville operator

$$
\mathcal{L}_{\text {ad }} \varrho=\frac{\kappa U_{0}^{2} J^{2} \eta^{2}}{\left(\kappa^{2}+\delta_{C}^{2}\right)^{2}}\left(2 \hat{B} \varrho \hat{B}-\hat{B}^{2} \varrho-\varrho \hat{B}^{2}\right),
$$

which describes decoherence in the basis of the eigenstates of the operator $\hat{B}$. Note that the adiabatic elimination procedure described above is not rigorous mathematically, since we adiabatically approximated the solution of a nonlinear dynamical equation in Eq. (75), which appears also as an ordering ambiguity of the involved operators (Larson et al., 2008b; Maschler et al., 2008).

For a small, numerically tractable system, the lowest energy eigenstate of $H_{\mathrm{ad}}$ can be calculated. As a key example, the SF-to-MI quantum phase transition in an optical lattice sustained entirely by a quantized standingwave cavity field was analyzed (Maschler et al., 2008). The dynamical response of the photon number to the atomic motion is able to strongly modify atomic number fluctuations and hence to drive the phase transition. Depending on the cavity parameters (e.g. the detuning between cavity and external pump laser), the photon fluctuations can either suppress or enhance atomic fluctuations and hopping, therefore pushing the system towards or outwards the MI or SF states. Accordingly, as depicted in Fig. 43, the position of the SF-to-MI phase transition in a cavity optical lattice potential can be shifted (keeping the mean potential depth constant) towards either smaller or larger values of the collisional atom-atom interaction strengths, depending on whether the pump-cavity detuning is chosen positive (a) or negative (b).

Figure 44 demonstrates the importance of the photon number fluctuations in a quantum potential by testing the stability of the Mott phase in a potential of fixed average depth but different mean photon number. While in an almost classical field (highly excited coherent state
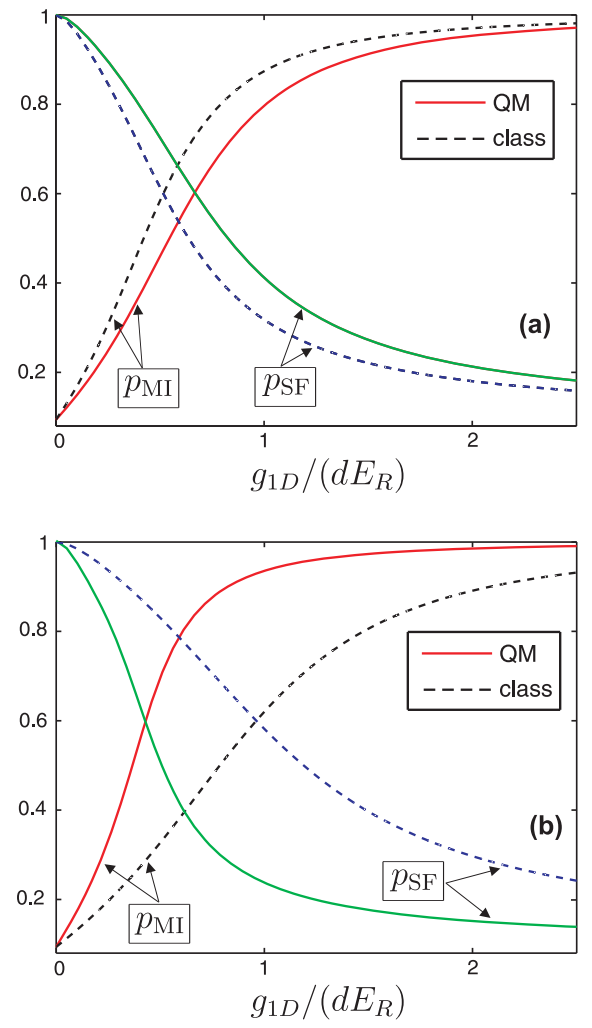

FIG. 43 Mott insulator (MI) to superfluid (SF) phase transition in a cavity optical lattice. The probabilities $p_{\mathrm{MI}}$ and $p_{\mathrm{SF}}$ to find the atoms in the states $\left|\Psi_{\mathrm{MI}}\right\rangle$ and $\left|\Psi_{\mathrm{SF}}\right\rangle$ as a function of the dimensionless $1 \mathrm{D}$ on-site interaction strength $g_{1 D} /\left(d E_{R}\right)$ ( $d$ is the lattice constant, $E_{R}=\hbar \omega_{R}$ is the recoil energy) are compared for two cases: first, for an optical lattice sustained by the quantum field of a cavity mode $\left(V_{\mathrm{cl}}=0\right)$, and second, for a purely classical optical lattice $\left(\eta_{1}=0\right)$. We choose $\eta$ such that in each of the two examples (a and b) both potentials have equal depth for zero on-site interaction $g_{1 \mathrm{D}}$. The quantum (QM) and classical (class) cases are depicted with solid and dashed lines, respectively. Parameters are $\left(U_{0}, \kappa, \eta\right)=(-1,1 / \sqrt{2}, \sqrt{5.5}) \omega_{R}$. The detuning between the probe and the dispersively shifted cavity frequencies affects the position of the phase transition. In (a) resp. (b) this detuning is positive, $\Delta_{C}-U_{0} N=\kappa$, resp. negative $\Delta_{C}-U_{0} N=-\kappa$ and the transition point is shifted towards smaller resp. higher interaction strengths in comparison to that in a classical lattice. From Maschler et al. (2008).

with many photons) the Mott phase is stable, photon number fluctuations (uncertainty) inherent in a weak coherent state of few photons enhance tunneling and decay of the perfect order.

This is explicitly shown in Fig. 44 depicting the decay of an initially prepared perfectly ordered atomic state. We choose different mean intracavity photon numbers and keep the average depth of the potential constant by readjusting the coupling strength $U_{0}$. In the classical limit (very large photon number and small atom-cavity coupling), the system remains in the initially prepared MI state. For smaller photon numbers $\bar{n} \sim 20$, the ini- 


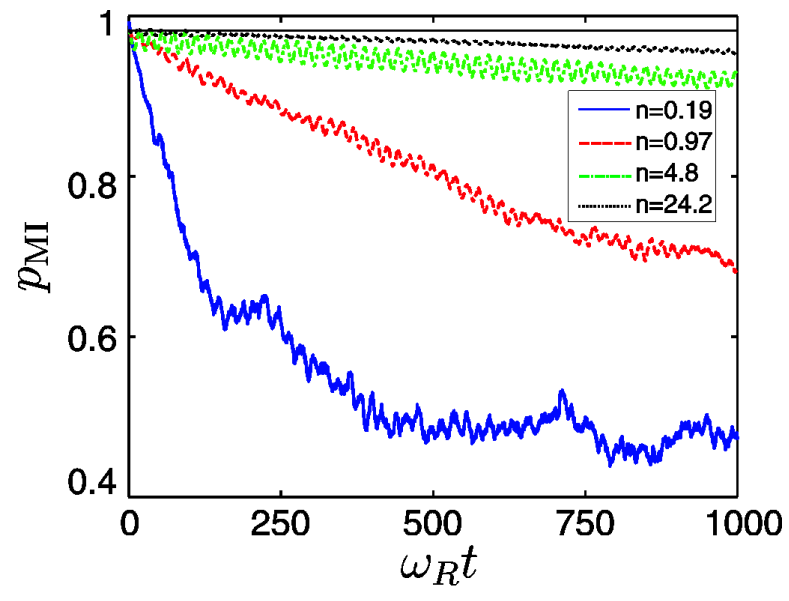

FIG. 44 (Color online) Effect of photon number granularity upon the Mott insulator (MI) state for two atoms in a cavitysustained optical lattice. The time evolution of the occupation probability $p_{\mathrm{MI}}$ of the MI state is shown for various mean intracavity photon numbers $n$. The atom-cavity coupling $g$ is adjusted such that the average potential depth $\left(8 E_{R}\right)$ is identical for all curves. Whereas for a static optical lattice potential of equal depth the system remains in the initially prepared MI state (solid line), photon number fluctuations for low $n$ deplete the MI state. From Maschler et al. (2008).

tial MI state only slowly degrades in time. However, when the photon number fluctuations become comparable to the mean, i.e. for mean photon numbers as small as $\bar{n} \sim 1$, the system quickly escapes from the MI state via fluctuation induced tunneling. Note that in order to keep the average optical potential constant, lower photon numbers are connected to a larger potential per photon, so that the potential fluctuations are additionally enhanced at low photon numbers. The classical limit is also approached in the bad cavity limit, $\kappa \gg \omega_{R}$, where number fluctuations occur so fast, that particles only see the average and do not have the time to tunnel during an intensity fluctuation.

The quantum properties of the cavity light become predominant if already single intracavity photons create an optical potential of considerable depth, capable of trapping numerous atoms. As quantum mechanics allows for the existence of superpositions of photon number states, one may obtain superpositions of several potentials with different depths (Horak and Ritsch, 2001b).

The complete phase diagram of ultracold atoms in twoband $\mathrm{BH}$ models coupled to a cavity light field has been calculated by Silver et al. (2010) by means of a variational approach and the analogy to the Dicke-model superradiant phase transition has been pointed out, see Sec. IV.D.2.

b. Self-organization within the Hubbard-model approach A possible influence of quantum statistical properties on the (a)
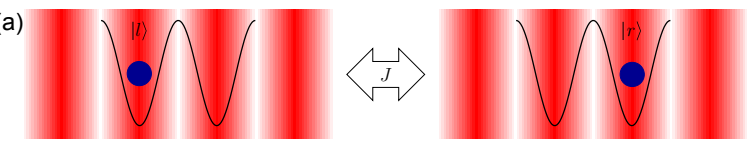

(b)

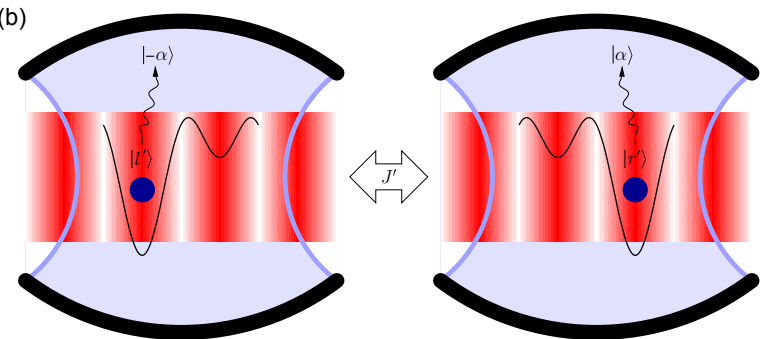

FIG. 45 Self-organization as a quantum seesaw effect. (a) Atoms which are trapped in a free-space 1D lattice potential with two adjacent sites (left and right) tunnel with rate $J$ between the corresponding Wannier states $|l\rangle$ and $|r\rangle$. (b) Coupling the atoms in addition to the field of a cavity whose axis is perpendicular to the optical lattice induces light scattering between the optical lattice laser and the cavity field with opposite phases from the two sites. The modified potential resulting from the interference of the lattice field and the cavity field discriminates the two sites and causes positive feedback and atomic ordering into one of them. The process starts by spontaneous symmetry breaking, and depends on the quantum statistics of the initially prepared many-body state.

spatial self-organization process, described in Sec. III.B.1 and IV.D, can be studied within the framework of an extended BH model. For simplicity, the geometry is modified in comparison with the generic case of selforganization, as depicted schematically in Fig. 45. Atoms are confined in a static optical lattice potential which is oriented perpendicular to the cavity axis. As before, large atom-laser detuning and negligible atomic saturation are assumed. The laser fields providing the optical lattice potential are considered to be tuned close to resonance with a cavity mode, therefore inducing coherent cavity driving via Rayleigh scattering off the atoms. The single-atom Hamiltonian corresponding to this geometry reads (Maschler et al., 2007)

$$
\begin{aligned}
H=\frac{p^{2}}{2 m}+V_{\mathrm{cl}} \cos ^{2}(k x)-\hbar\left(\Delta_{C}-U_{0}\right) a^{\dagger} a \\
+\sqrt{\hbar V_{\mathrm{cl}} U_{0}} \cos (k x)\left(a+a^{\dagger}\right),
\end{aligned}
$$

where $V_{\mathrm{cl}}$ denotes the depth of the static lattice potential, $\Delta_{C}$ the detuning between the lattice laser and the cavity resonance, and $U_{0}$ the light shift of the cavity resonance frequency per atom. In a simple and intuitive picture the dynamic cavity field plays the role of a "seesaw" potential. Interference between the $\cos (k x)$ potential, generated through photon scattering, and the static $\cos ^{2}(k x)$ potential determines the overall potential felt by the atoms. The spatial symmetry of the system allows for the emergence of two possible ordered configurations, with all atoms residing at either odd $(\cos (k x)=1)$ or 


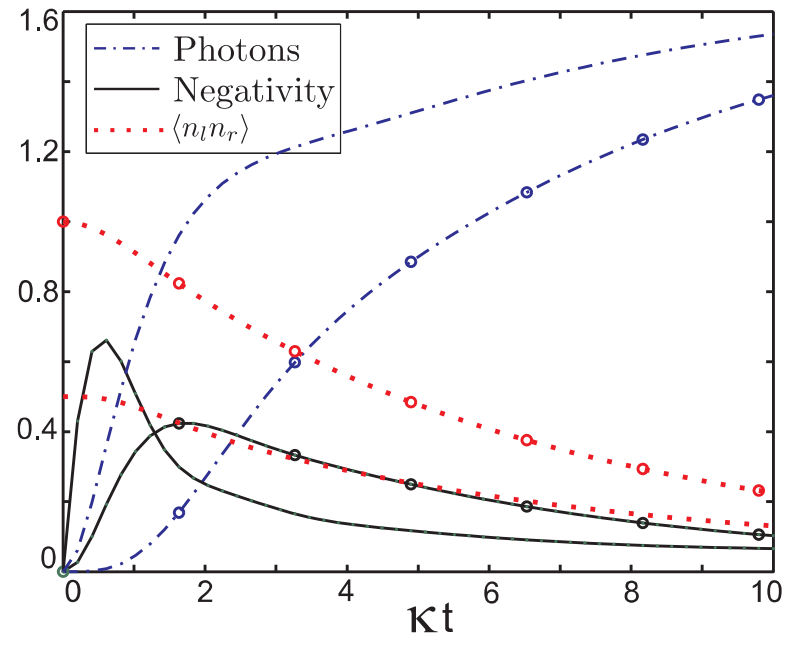

FIG. 46 Entanglement-assisted self-organization in a quantum optical lattice potential, see Fig. 45. Shown are atomlight entanglement (solid lines), mean cavity photon number (dotted-dashed lines), and the two-site atom-atom correlation function (dotted lines) for two atoms in two adjacent lattice wells, denoted by left $(l)$ and right $(r)$. Lines with extra circles show the case of exactly one atom in each well at start (MI state), while the other lines show the evolution for an initially symmetric superposition state for each atom (SF state). The parameters are $U_{0}=-2 \kappa, \Delta_{C}=-6 \kappa, J=\kappa / 100$ and $\tilde{J}=1.6 \kappa$. From Maschler et al. (2007).

even $(\cos (k x)=-1)$ lattice sites.

The many-body Bose-Hubbard Hamiltonian, Eq. (70), adapted to this scheme reads

$$
\begin{aligned}
H=\sum_{i, j} J_{i, j} b_{i}^{\dagger} b_{j}-\hbar( & \left.\Delta_{C}-U_{0} \sum_{i} b_{i}^{\dagger} b_{i}\right) a^{\dagger} a \\
& +\left(a+a^{\dagger}\right) \sum_{i, j} \hbar \tilde{J}_{i, j} b_{i}^{\dagger} b_{j} .
\end{aligned}
$$

Here, the standard matrix elements for the kinetic and potential energy $p^{2} / 2 m+V_{\mathrm{cl}} \cos ^{2}(k x)$ between sites $i$ and $j$ are denoted by $J_{i, j}$, whereas $\tilde{J}_{i, j}$ gives the matrix elements of the interference term $\sqrt{U_{0} V_{\mathrm{cl}} / \hbar} \cos (k x)$. On-site interactions are neglected at this point and, for consistency, we require weak coupling per atom, i.e. $\hbar\left|U_{0}\right| \ll\left|V_{\mathrm{cl}}\right|$.

Essential dynamical properties of this system beyond the mean-field approximation become evident already for two atoms. Monitoring the microscopic physics of selforganization as shown in Fig. 46, the process resembles the decay of a homogeneously filled lattice with one particle per site on average to the self-ordered state, where both particles occupy even or odd sites. Following the decay of the probability for the two atoms sitting in different wells $\left(\left\langle n_{l} n_{r}\right\rangle\right)$, we first note that the formation of the self-organized state is accompanied by a fast growth of atom-field entanglement. Most importantly, however, one finds a striking dependence of the self-organization dynamics on the initial quantum fluctuations. A SF state with both atoms prepared in the symmetric superposition of the two wells, $1 / 2\left(b_{l}^{\dagger}+b_{r}^{\dagger}\right)^{2}|0\rangle$, self-organizes much faster than a perfectly ordered MI state, $b_{l}^{\dagger} b_{r}^{\dagger}|0\rangle$, with exactly one atom per well. In the latter case, the cavity field remains in the vacuum state until a tunneling event induces atomic coherence between the left and right lattice site, triggering the decay of the MI state towards the self-organized state.

Under some approximations this model can be extrapolated to the thermodynamic limit where quantum phase transitions similar to the one predicted in the mean-field approach can be studied. Among various other properties this leads to the coexistence of diagonal long-range order and long-range coherence (Vidal et al., 2010), indicating new phases to appear in the gaps between Mott-like states with different integer filling factors.

c. Ring cavity When several independent cavity modes are dynamically interacting with the atoms, not only the depth but also the shape and the spatial periodicity of the potential can change. In the generic case of a ring cavity the depth and the longitudinal position of the lattice is dynamical. While, already in standard optical lattices the validity of the lowest-band assumption is often doubtful and corrections are necessary, this approximation loses its meaning in a ring cavity. Expansions based on a single set of Wannier functions cannot be consistently formulated since the lowest-band Wannier functions for a given position contain contributions from a large number of higher bands for a slightly shifted position. Hence small lattice shifts immediately involve many higher-order bands.

The simplest example of two cavity modes sustained by a ring cavity reveals, that a naive crude truncation of the Bose-Hubbard model with respect to the pumped cosine mode at the lowest band decouples the atoms from the associated sine mode. This immediately eliminates central dynamical effects of the system as overall momentum conservation and nonlocal correlated hopping (Niedenzu et al., 2010).

As an example, Fig. 47 shows that a quantum jump in the lattice photon number is accompanied by a sudden change of the tunneling rate between adjacent sites. After the second jump in the trajectory shown in the Figure, the system returns to the original mean values, however, both the position and photon number quantities exhibit much larger noise, which demonstrates the effect of heating stemming from the photon number fluctuations. 


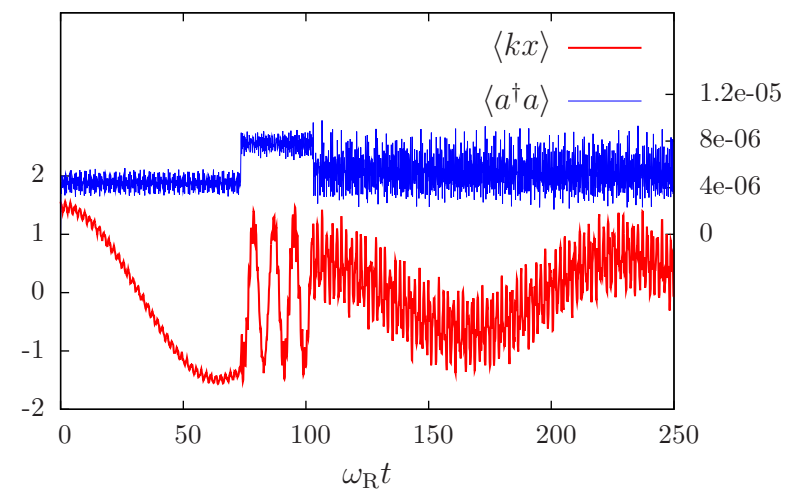

FIG. 47 (Color online) Correlated photon jumps and tunneling of an atom in a symmetrically driven ring cavity. The sample trajectory, showing the position expectation value $\langle k x\rangle$ (left axis) and the mean photon number $\left\langle a^{\dagger} a\right\rangle$ of the undriven sine mode (right axis), presents two quantum jumps occurring at $\omega_{R} t \approx 70$ and $\omega_{R} t \approx 100$. They lead to a simultaneous change of the photon number and the atomic band excitation corresponding to different tunnel oscillation frequencies. In a higher band much faster oscillations are obtained between neighboring sites. This generates a higher effective hopping amplitude and heating on average. The parameters are $U_{0}=-2 \omega_{R}, \alpha_{c}=\sqrt{6}$ (field amplitude of the driven cosine mode), $\Delta_{C}=U_{0}-\kappa$ and $\kappa=500 \omega_{R}$. From Niedenzu et al. (2010).

\section{OUTLOOK}

After almost two decades of active research in optical cavity QED with cold and ultracold atoms, initially dominated by theoretical investigations, the field presently exhibits fast growth with several experimental groups demonstrating spectacular effects. Single atoms are routinely cavity-cooled and trapped over seconds within optical high-finesse resonators, providing a well-controlled quantum system for quantum information science. Ultracold quantum gases prepared in magnetic or optical traps are now reliably coupled to high-quality cavity modes. Even in the far dispersive regime, these systems are governed by strong back-action effects of the collective atomic motion on the cavity field degrees of freedom. Cavity decay offers a unique channel to monitor the complex coupled atom-light dynamics non-destructively and in real time. Many central atomic variables can be accessed by quantum non-demolition measurements which minimize quantum back-action.

With the aim of trapping and cooling ensembles, nanoand even microscopic particles or arrays of thin membranes, the research field of cavity QED, reviewed in this paper, overlaps and unifies more and more with the rapidly growing field of optomechanics (Stamper-Kurn, 2012). Trapping and cooling arrays of membranes in cavities is only one very striking example (Xuereb et al., 2012). Practical applications as ultra-sensitive detectors of mass, acceleration or magnetic fields or even tests of general relativity seem within range of current technology.

Initiated by theoretical studies and early experiments with cold atomic ensembles, a non-equilibrium quantum phase transition between a superfluid and a supersolid phase has been investigated experimentally. Recently, theoretical investigations opened new directions and possibilities towards controlled preparation and investigations of the physics of spin glasses, more complex supersolid and superglass phases (Gopalakrishnan et al., 2011b; Strack and Sachdev, 2011). Further prominent solid-state Hamiltonians involving phonons or polarons could be studied with unprecedented control and observation possibilities (Mekhov and Ritsch, 2012a).

A recent breakthrough experiment demonstrating subrecoil cavity cooling towards quantum degeneracy (Wolke et al., 2012) opens the prospect of replacing evaporative cooling techniques by cavity cooling and direct preparation of exotic quantum states from a thermal gas. This also paves the way towards implementing a continuous atom-laser as a new tool in ultracold atom physics (Salzburger and Ritsch, 2007).

Still, important challenges as cooling and trapping of molecular samples or large suspended objects have not been experimentally demonstrated. The prospects of multi-species implementations in multimode cavity environments still have to be fully evaluated. Apart from this point, experiments seem ahead of theoretical and numerical simulation possibilities, where theory has to be improved and better suited models need to be developed.

In a long term vision, cavity-sustained light fields allow to couple hybrid systems of very different physical nature like superconducting qubits, cold quantum gases and micromechanical oscillators without destroying quantum coherence of the systems, brought about by any classical coupling of such systems. In this way cavity-based setups with ultracold gases could develop into an important building block for quantum information processing or other quantum-based future technologies (Henschel et al., 2010) or a route to an even better atomic lattice clock (Nicholson et al., 2012).

\section{Acknowledgments}

We thank D. Nagy, G. Szirmai, G. Kónya, A. Vukics, J. Asbóth, I. Mekhov, C. Genes, K. Baumann, R. Mottl, T. Donner and R. Landig for stimulating discussions. H. R. acknowledges support from the Austrian Science Fund FWF (grant F 4013-N16). P. D. acknowledges the financial support from the Hungarian National Office for Research and Technology under the contract ERC_HU_09 OPTOMECH, and from the Hungarian Academy of Sciences (Lendület Program, LP2011-016). F. B. and T. E. 
acknowledge financial funding from SQMS (ERC advanced grant), NAME-QUAM (EU, FET open), NCCRQSIT and ESF (POLATOM).

\section{REFERENCES}

Arcizet, O., P. F. Cohadon, T. Briant, M. Pinard, and A. Heidmann (2006), Nature 444 (7115), 71.

Asbóth, J. K., and P. Domokos (2007), Physical Review A (Atomic, Molecular, and Optical Physics) 76 (5), 057801+.

Asbóth, J. K., P. Domokos, and H. Ritsch (2004), Physical Review A 70 (1), 013414+.

Asbóth, J. K., P. Domokos, H. Ritsch, and A. Vukics (2005), Physical Review A 72 (5), 053417+.

Barker, P. F., and M. N. Shneider (2010), Physical Review A 81 (2), 023826+.

Baumann, K., C. Guerlin, F. Brennecke, and T. Esslinger (2010), Nature 464 (7293), 1301.

Baumann, K., R. Mottl, F. Brennecke, and T. Esslinger (2011), Physical Review Letters 107, 140402+.

Berman, P. R. (1994), Advances in atomic, molecular, and optical physics, New York: Academic Press, edited by Berman, Paul R.

Berman, P. R. (1999), Phys. Rev. A 59, 585.

Bhaseen, M. J., J. Mayoh, B. D. Simons, and J. Keeling (2012), Physical Review A 85, 013817+.

Bhattacherjee, A. B., T. Kumar, and M. Mohan (2010), Central European Journal of Physics 8 (5), 850.

Black, A. T., H. W. Chan, and V. Vuletić (2003), Physical Review Letters 91 (20), 203001+.

Blake, T., A. Kurcz, and A. Beige (2011), Journal of Modern Optics 58 (15), 1317.

Bloch, I., J. Dalibard, and W. Zwerger (2008), Reviews of Modern Physics 80 (3), 885.

Boca, A., R. Miller, K. M. Birnbaum, A. D. Boozer, J. McKeever, and H. J. Kimble (2004), Physical Review Letters 93 (23), 233603+.

Bonifacio, R. (1996), Optics Communications 124 (5-6), 469.

Bonifacio, R., L. De Salvo, L. M. Narducci, and E. J. D'Angelo (1994), Physical Review A 50 (2), 1716.

Boozer, A. D., A. Boca, R. Miller, T. E. Northup, and H. J. Kimble (2006), Physical Review Letters 97 (8), 083602+.

Boozer, A. D., A. Boca, R. Miller, T. E. Northup, and H. J. Kimble (2007), Physical Review Letters 98 (19), 193601+. Botter, T., D. Brooks, S. Gupta, M. A. Zhao-Yuan, K. L. Moore, K. W. Murch, T. Purdy, and D. M. Stamper Kurn (2009), in 21st International Conference on Atomic Physics (World Scientific Pub Co Inc) p. 117, arXiv:arXiv:0810.3841v1.

Brahms, N., T. Botter, S. Schreppler, D. W. C. Brooks, and D. M. Stamper Kurn (2012), Physical Review Letters 108, $133601+$

Brahms, N., and D. M. S. Kurn (2010), Physical Review A $82(4), 041804+$.

Brazovskii, S. (1975), Sov Phys JETP 41 (1), 85.

Brennecke, F., T. Donner, S. Ritter, T. Bourdel, M. Köhl, and T. Esslinger (2007), Nature 450 (7167), 268.

Brennecke, F., S. Ritter, T. Donner, and T. Esslinger (2008), Science 322 (5899), 235.

Brooks, D. W. C., T. Botter, S. Schreppler, T. P. Purdy, N. Brahms, and D. M. Stamper-Kurn (2012), Nature 488 (7412), 476.
Brune, M., S. Haroche, V. Lefevre, J. M. Raimond, and N. Zagury (1990), Physical Review Letters 65, 976.

Brzozowska, M., T. M. Brzozowski, J. Zachorowski, and W. Gawlik (2006), Physical Review A 73, 063414+.

Büchler, H. P., and G. Blatter (2003), Physical Review Letters 91, 130404+.

Bux, S., C. Gnahm, R. A. W. Maier, C. Zimmermann, and P. W. Courteille (2011), Physical Review Letters 106, $203601+$.

Carmichael, H. J. (2003), Statistical Methods in Quantum Optics 1: Master Equations and Fokker-Planck Equations (Springer).

Carmichael, H. J., C. W. Gardiner, and D. F. Walls (1973), Physics Letters A 46 (1), 47.

Caves, C. M. (1980), Physical Review Letters 45 (2), 75.

Chan, H. W., A. T. Black, and V. Vuletić (2003), Physical Review Letters 90 (6), 063003+.

Chang, D. E., C. A. Regal, S. B. Papp, D. J. Wilson, J. Ye, O. Painter, H. J. Kimble, and P. Zoller (2009), Proceedings of the National Academy of Sciences 107 (3), 1005.

Chavanis, P. (2007), Physica A: Statistical Mechanics and its Applications 377 (2), 469.

Chen, B., C. Jiang, J. J. Li, and K. Di Zhu (2011), Physical Review A 84, 055802+.

Chen, W., D. S. Goldbaum, M. Bhattacharya, and P. Meystre (2010), Physical Review A 81 (5), 053833+.

Chen, W., D. Meiser, and P. Meystre (2007), Physical Review A 75, 023812+.

Chen, W., and P. Meystre (2009), Physical Review A 79 (4), $043801+$.

Chen, W., K. Zhang, D. S. Goldbaum, M. Bhattacharya, and P. Meystre (2009), Physical Review A 80 (1), 011801+.

Chu, S. (1998), Reviews of Modern Physics 70 (3), 685.

Cirac, J. I., M. Lewenstein, and P. Zoller (1995), Physical Review A 51 (2), 1650.

Cirac, J. I., P. Zoller, H. J. Kimble, and H. Mabuchi (1997), Physical Review Letters 78 (16), 3221.

Cohen-Tannoudji, C. (1992), in Fundamental Systems in Quantum Optics, Proceedings of the Les Houches Summer School, Session LIII, edited by J. Dalibard, J.-M. Raimond, and J. Zinn-Justin (North-Holland, Amsterdam) pp. 1-164.

Cohen Tannoudji, C. N. (1998), Reviews of Modern Physics $70(3), 707$.

Colombe, Y., T. Steinmetz, G. Dubois, F. Linke, D. Hunger, and J. Reichel (2007), Nature, Nature 450 (7167), 272.

Cornell, E. A., and C. E. Wieman (2002), Reviews of Modern Physics 74 (3), 875.

Courtois, J.-Y., G. Grynberg, B. Lounis, and P. Verkerk (1994), Phys. Rev. Lett. 72, 3017.

Cube, C., S. Slama, D. Kruse, C. Zimmermann, P. W. Courteille, G. R. M. Robb, N. Piovella, and R. Bonifacio (2004), Phys. Rev. Lett. 93, 083601+.

Dalibard, J., and C. Cohen-Tannoudji (1985), Journal of Physics B: Atomic and Molecular Physics 18 (8), 1661+.

De Chiara, G., M. Paternostro, and G. M. Palma (2011), Physical Review A 83 (5), 052324+.

Deachapunya, S., P. J. Fagan, A. G. Major, E. Reiger, H. Ritsch, A. Stefanov, H. Ulbricht, and M. Arndt (2008), The European Physical Journal D - Atomic, Molecular, Optical and Plasma Physics 46 (2), 307.

DeVoe, R. G., and R. G. Brewer (1996), Physical Review Letters 76, 2049.

Dicke, R. H. (1954), Physical Review Online Archive (Prola) 93 (1), 99. 
Dimer, F., B. Estienne, A. S. Parkins, and H. J. Carmichael (2007), Physical Review A 75 (1), 013804+.

Doherty, A. C., T. W. Lynn, C. J. Hood, and H. J. Kimble (2000), Physical Review A 63 (1), 013401+.

Domokos, P., P. Horak, and H. Ritsch (2001), J. Phys. B 34, $187+$.

Domokos, P., and H. Ritsch (2002), Physical Review Letters 89 (25), 253003+.

Domokos, P., and H. Ritsch (2003), J. Opt. Soc. Am. B 20 (5), 1098.

Domokos, P., T. Salzburger, and H. Ritsch (2002), Physical Review A 66 (4), 043406+.

Domokos, P., A. Vukics, and H. Ritsch (2004), Physical Review Letters 92 (10), 103601+.

Dotsenko, I., W. Alt, M. Khudaverdyan, S. Kuhr, D. Meschede, Y. Miroshnychenko, D. Schrader, and A. Rauschenbeutel (2005), Physical Review Letters 95 (3), $033002+$.

Elsasser, T., B. Nagorny, and A. Hemmerich (2003), Physical Review A 67 (5), 051401+.

Elsasser, T., B. Nagorny, and A. Hemmerich (2004), Physical Review A 69 (3), 033403+.

van Enk, S. J., J. McKeever, H. J. Kimble, and J. Ye (2001), Physical Review A 64 (1), 013407+.

Fabre, C., M. Pinard, S. Bourzeix, A. Heidmann, E. Giacobino, and S. Reynaud (1994), Physical Review A 49 (2), 1337.

Figueroa, E., M. Mücke, J. Bochmann, C. Hahn, K. Murr, S. Ritter, C. Villas-Boas, and G. Rempe (2011), in AIP Conference Proceedings, Vol. 1363, p. 389.

Fischer, T., P. Maunz, P. W. H. Pinkse, T. Puppe, and G. Rempe (2002), Physical Review Letters 88 (16), $163002+$.

Fischer, T., P. Maunz, T. Puppe, P. W. H. Pinkse, and G. Rempe (2001), New Journal of Physics 3 (1), 11.

Fisher, M. P. A., P. B. Weichman, G. Grinstein, and D. S. Fisher (1989), Physical Review B 40 (1), 546.

Gangl, M., P. Horak, and H. Ritsch (2000), Journal of Modern Optics 47 (14-15), 2741.

Gangl, M., and H. Ritsch (1999), Physical Review A 61 (1), $011402+$.

Gangl, M., and H. Ritsch (2000), Physical Review A 61 (4), 043405+.

Gangl, M., and H. Ritsch (2001), Physical Review A 64 (6), 063414+.

Gardiner, C. W., and P. Zoller (2004), Quantum Noise: A Handbook Of Markovian And Non-markovian Quantum Stochastic Methods with Applications to Quantum Optics, third edition ed. (Springer Berlin / Heidelberg).

Gardiner, S. A., K. M. Gheri, and P. Zoller (2001), Physical Review A 63 (5), 051603+.

Genes, C., H. Ritsch, and D. Vitali (2009), Physical Review A 80 (6), 061803+.

Genes, C., D. Vitali, P. Tombesi, S. Gigan, and M. Aspelmeyer (2008), Physical Review A (Atomic, Molecular, and Optical Physics) 77 (3), 033804+.

Gigan, S., H. R. Bohm, M. Paternostro, F. Blaser, G. Langer, J. B. Hertzberg, K. C. Schwab, D. Bauerle, M. Aspelmeyer, and A. Zeilinger (2006), Nature 444 (7115), 67.

Giorgini, S., L. P. Pitaevskii, and S. Stringari (2008), Reviews of Modern Physics 80 (4), 1215.

Gopalakrishnan, S., B. L. Lev, and P. M. Goldbart (2009), Nature Physics 5 (11), 845.

Gopalakrishnan, S., B. L. Lev, and P. M. Goldbart (2010),
Physical Review A 82 (4), 043612+.

Gopalakrishnan, S., B. L. Lev, and P. M. Goldbart (2011a), Philosophical Magazine 92 (1-3), 353.

Gopalakrishnan, S., B. L. Lev, and P. M. Goldbart (2011b), Physical Review Letters 107, 277201+.

Gordon, J. P., and A. Ashkin (1980), Physical Review A 21 (5), 1606.

Grangier, P., and Poizat (1998), The European Physical Journal D - Atomic, Molecular, Optical and Plasma Physics 1 (1), 97.

Grießer, T., W. Niedenzu, and H. Ritsch (2011), arXiv:1106.2340.

Grießer, T., and H. Ritsch (2011), Opt. Express 19 (12), 11242.

Grießer, T., H. Ritsch, M. Hemmerling, and Robb (2010), The European Physical Journal D - Atomic, Molecular, Optical and Plasma Physics 58 (3), 349.

Grimm, R., M. Weidemüller, and Y. B. Ovchinnikov (2000), "Optical Dipole Traps for Neutral Atoms," (Elsevier) pp. 95-170.

Gross, M., and S. Haroche (1982), Physics Reports 93 (5), 301.

Guerlin, C., J. Bernu, S. Deleglise, C. Sayrin, S. Gleyzes, S. Kuhr, M. Brune, J.-M. Raimond, and S. Haroche (2007), Nature 448 (7156), 889.

Gupta, S., K. L. Moore, K. W. Murch, and D. M. StamperKurn (2007), Physical Review Letters 99 (21), 213601+.

Happer, W. (1972), Reviews of Modern Physics 44 (2), 169.

Haroche, S. (1992), in Fundamental Systems in Quantum Optics, Proceedings of the Les Houches Summer School, Session LIII, edited by J. Dalibard, J.-M. Raimond, and J. Zinn-Justin (North-Holland, Amsterdam) p. 165.

Hechenblaikner, G., M. Gangl, P. Horak, and H. Ritsch (1998), Physical Review A 58 (4), 3030.

Henschel, K., J. Majer, J. Schmiedmayer, and H. Ritsch (2010), Physical Review A 82, 033810+.

Hepp, K., and E. H. Lieb (1973), Annals of Physics 76 (2), 360.

Herskind, P. F., A. Dantan, J. P. Marler, M. Albert, and M. Drewsen (2009), Nat Phys 5 (7), 494.

Hood, C. J., M. S. Chapman, T. W. Lynn, and H. J. Kimble (1998), Physical Review Letters 80 (19), 4157.

Hood, C. J., T. W. Lynn, A. C. Doherty, A. S. Parkins, and H. J. Kimble (2000), Science 287 (5457), 1447.

Horak, P., S. M. Barnett, and H. Ritsch (2000), Physical Review A 61 (3), 033609+.

Horak, P., G. Hechenblaikner, K. M. Gheri, H. Stecher, and H. Ritsch (1997), Physical Review Letters 79 (25), 4974.

Horak, P., and H. Ritsch (2001a), Physical Review A 63 (2), $023603+$.

Horak, P., and H. Ritsch (2001b), The European Physical Journal D - Atomic, Molecular, Optical and Plasma Physics 13 (2), 279.

Horak, P., and H. Ritsch (2001c), Physical Review A 64 (3), $033422+$.

Horak, P., H. Ritsch, T. Fischer, P. Maunz, T. Puppe, P. W. H. Pinkse, and G. Rempe (2002), Physical Review Letters 88 (4), 043601+.

Imamoglu, A., H. Schmidt, G. Woods, and M. Deutsch (1997), Physical Review Letters 79 (8), 1467.

Inouye, S., A. P. Chikkatur, D. M. Stamper-Kurn, J. Stenger, D. E. Pritchard, and W. Ketterle (1999), Science 285 (5427), 571.

Jayich, A., J. Sankey, A. Petrenko, and J. Harris (2011), in 
Quantum Electronics and Laser Science Conference (Optical Society of America) p. QThM3.

Jayich, A. M., J. C. Sankey, B. M. Zwickl, C. Yang, J. D. Thompson, S. M. Girvin, A. A. Clerk, F. Marquardt, and J. G. E. Harris (2008), New Journal of Physics 10 (9), 095008+.

Jaynes, E. T., and F. W. Cummings (1963), Proceedings of the IEEE 51 (1), 89+.

Jing, H., D. S. Goldbaum, L. Buchmann, and P. Meystre (2011), Physical Review Letters 106 (22), 223601+.

Kampschulte, T., W. Alt, S. Brakhane, M. Eckstein, R. Reimann, A. Widera, and D. Meschede (2010), Physical Review Letters 105 (15), 153603+.

Kanamoto, R., and P. Meystre (2010), Physical Review Letters 104 (6), 063601+.

Keeling, J., J. Bhaseen, and B. Simons (2010a), Physics 3, 88.

Keeling, J., M. J. Bhaseen, and B. D. Simons (2010b), Physical Review Letters 105 (4), 043001+.

Keller, M., B. Lange, K. Hayasaka, W. Lange, and H. Walther (2004), Nature 431, 1075.

Ketterle, W. (2002), Reviews of Modern Physics 74 (4), 1131.

Khudaverdyan, M., W. Alt, I. Dotsenko, T. Kampschulte, K. Lenhard, A. Rauschenbeutel, S. Reick, K. Schörner, A. Widera, and D. Meschede (2008), New Journal of Physics 10 (7), 073023+.

Kimble, H. J. (1998), Physica Scripta T76, 127.

Kippenberg, T. J., and K. J. Vahala (2008), Science (New York, N.Y.) 321 (5893), 1172.

Kleckner, D., and D. Bouwmeester (2006), Nature 444 (7115), 75.

Klinner, J., M. Lindholdt, B. Nagorny, and A. Hemmerich (2006), Physical Review Letters 96 (2), 023002+.

Kónya, G., G. Szirmai, and P. Domokos (2011), The European Physical Journal D - Atomic, Molecular, Optical and Plasma Physics 65 (1), 33.

Kowalewski, M., G. Morigi, P. W. H. Pinkse, and R. de VivieRiedle (2007), Applied Physics B 89 (4), 459.

Kowalewski, M., G. Morigi, P. W. H. Pinkse, and R. de Vivie Riedle (2011), Physical Review A 84, 033408+.

Kruse, D., C. von Cube, C. Zimmermann, and P. W. Courteille (2003a), Phys. Rev. Lett. 91, 183601.

Kruse, D., M. Ruder, J. Benhelm, C. von Cube, C. Zimmermann, Ph, Th, B. Nagorny, and A. Hemmerich (2003b), Physical Review A 67 (5), 051802+.

Kubanek, A., M. Koch, C. Sames, A. Ourjoumtsev, P. W. H. Pinkse, K. Murr, and G. Rempe (2009), Nature 462 (7275), 898.

Kubanek, A., M. Koch, C. Sames, A. Ourjoumtsev, T. Wilk, P. Pinkse, G. Rempe, A. Kubanek, M. Koch, C. Sames, A. Ourjoumtsev, T. Wilk, P. W. H. Pinkse, and G. Rempe (2011), Applied Physics B: Lasers and Optics 102 (3), 433.

Kubanek, A., A. Ourjoumtsev, I. Schuster, M. Koch, P. W. H. Pinkse, K. Murr, and G. Rempe (2008), Physical Review Letters 101 (20), 203602+.

Kuhn, A., M. Hennrich, and G. Rempe (2002), Physical Review Letters 89 (6), 067901+.

Kuhr, S., W. Alt, D. Schrader, I. Dotsenko, Y. Miroshnychenko, W. Rosenfeld, M. Khudaverdyan, V. Gomer, A. Rauschenbeutel, and D. Meschede (2003), Physical Review Letters 91 (21), 213002+.

Kuramoto, Y. (1975), in International Symposium on Mathematical Problems in Theoretical Physics, Lecture Notes in Physics, Vol. 39, edited by H. Araki (Springer Berlin /
Heidelberg) pp. 420-422, 10.1007/BFb0013365.

Lambert, N., C. Emary, and T. Brandes (2004), Physical Review Letters 92 (7), 073602+.

Larson, J., B. Damski, G. Morigi, and M. Lewenstein (2008a), Physical Review Letters 100 (5), 050401+.

Larson, J., S. Fernández-Vidal, G. Morigi, and M. Lewenstein (2008b), New Journal of Physics 10 (4), 045002+.

Larson, J., and M. Lewenstein (2009), New Journal of Physics 11 (6), 063027+.

Larson, J., G. Morigi, and M. Lewenstein (2008c), Physical Review A 78 (2), 023815.

Leggett, A. J. (1970), Physical Review Letters 25 (22), 1543.

Leibrandt, D. R., J. Labaziewicz, V. Vuletić, and I. L. Chuang (2009), Physical Review Letters 103 (10), $103001+$.

Lev, B. L., A. Vukics, E. R. Hudson, B. C. Sawyer, P. Domokos, H. Ritsch, and J. Ye (2008), Physical Review A (Atomic, Molecular, and Optical Physics) 77 (2), $023402+$.

Lewenstein, M., and L. Roso (1993), Physical Review A 47 (4), 3385.

Lewenstein, M., A. Sanpera, V. Ahufinger, B. Damski, A. SenDe, and U. Sen (2007), Advances in Physics 56 (2), 243.

Liu, N., J. Lian, J. Ma, L. Xiao, G. Chen, J. Q. Liang, and S. Jia (2011), Physical Review A 83 (3), 033601+.

Lu, W., Y. Zhao, and P. F. Barker (2007), Physical Review A 76 (1), 013417+.

Luciani, J., and R. Pellat (1987), Journal de Physique 48 (4), 591.

Ludwig, M., B. Kubala, and F. Marquardt (2008), New Journal of Physics 10 (9), 095013+.

Lugiato, L. A. (1984), "II Theory of Optical Bistability," (Elsevier) pp. 69-216.

Mabuchi, H., and A. C. Doherty (2002), Science 298 (5597), 1372.

Mabuchi, H., Q. A. Turchette, M. S. Chapman, and H. J. Kimble (1996), Opt. Lett. 21 (17), 1393.

Mancini, S., and P. Tombesi (1994), Physical Review A 49, 4055 .

Marquardt, F., J. P. Chen, A. A. Clerk, and S. M. Girvin (2007), Physical Review Letters 99 (9), 093902+.

Maschler, C., I. B. Mekhov, and H. Ritsch (2008), The European Physical Journal D - Atomic, Molecular, Optical and Plasma Physics 46 (3), 545.

Maschler, C., and H. Ritsch (2005), Physical Review Letters 95 (26), 260401+.

Maschler, C., H. Ritsch, A. Vukics, and P. Domokos (2007), Optics Communications 273 (2), 446.

Maunz, P., T. Puppe, T. Fischer, P. W. H. Pinkse, and G. Rempe (2003), Opt. Lett. 28 (1), 46.

Maunz, P., T. Puppe, I. Schuster, N. Syassen, P. W. H. Pinkse, and G. Rempe (2004), Nature 428 (6978), 50.

Maunz, P., T. Puppe, I. Schuster, N. Syassen, P. W. H. Pinkse, and G. Rempe (2005), Physical Review Letters 94 (3), 033002+.

McKeever, J., A. Boca, A. D. Boozer, R. Miller, J. R. Buck, A. Kuzmich, and H. J. Kimble (2004), Science 303 (5666), 1992.

McKeever, J., J. R. Buck, A. D. Boozer, A. Kuzmich, H. C. Nägerl, D. M. S. Kurn, and H. J. Kimble (2003), Physical Review Letters 90 (13), 133602+.

Mekhov, I., and H. Ritsch (2012a), Arxiv preprint arXiv:1203.0552. 
Mekhov, I. B., C. Maschler, and H. Ritsch (2007a), Physical Review Letters 98 (10), 100402+.

Mekhov, I. B., C. Maschler, and H. Ritsch (2007b), Physical Review A 76 (5), 053618+.

Mekhov, I. B., C. Maschler, and H. Ritsch (2007c), Nat Phys 3 (5), 319.

Mekhov, I. B., and H. Ritsch (2009), Physical Review A 80 (1), 013604+.

Mekhov, I. B., and H. Ritsch (2012b), Journal of Physics B: Atomic, Molecular and Optical Physics 45 (10), 102001+.

Metzger, C. H., and K. Karrai (2004), Nature 432 (7020), 1002.

Miyake, H., G. A. Siviloglou, G. Puentes, D. E. Pritchard, W. Ketterle, and D. M. Weld (2011), Physical Review Letters 107, 175302+.

Montgomery, D. C. (1971), Theory of the Unmagnetized Plasma (Gordon and Breach).

Moore, M. G., O. Zobay, and P. Meystre (1999), Physical Review A 60 (2), 1491.

Morigi, G., J. Eschner, and C. H. Keitel (2000), Physical Review Letters 85 (21), 4458.

Morigi, G., P. W. H. Pinkse, M. Kowalewski, and R. de Vivie Riedle (2007), Physical Review Letters 99 (7), 073001+.

Mossberg, T. W., M. Lewenstein, and D. J. Gauthier (1991), Physical Review Letters 67 (13), 1723.

Mottl, R., F. Brennecke, K. Baumann, R. Landig, T. Donner, and T. Esslinger (2012), Science 336 (6088), 1570.

Müller, M., P. Strack, and S. Sachdev (2012), Physical Review A 86, 023604+.

Münstermann, P., T. Fischer, P. Maunz, P. W. H. Pinkse, and G. Rempe (1999), Physical Review Letters 82 (19), 3791.

Münstermann, P., T. Fischer, P. Maunz, P. W. H. Pinkse, and G. Rempe (2000), Physical Review Letters 84 (18), 4068.

Murch, K. W., K. L. Moore, S. Gupta, and D. M. StamperKurn (2008), Nature Physics 4 (7), 561.

Murr, K. (2003), Journal of Physics B: Atomic, Molecular and Optical Physics 36 (12), 2515.

Murr, K. (2006), Physical Review Letters 96 (25), 253001+.

Murr, K., P. Maunz, P. W. H. Pinkse, T. Puppe, I. Schuster, D. Vitali, and G. Rempe (2006a), Physical Review A 74 (4), 043412+.

Murr, K., S. Nussmann, T. Puppe, M. Hijlkema, B. Weber, S. C. Webster, A. Kuhn, and G. Rempe (2006b), Physical Review A 73 (6), 063415+.

Nagorny, B., T. Elsasser, and A. Hemmerich (2003), Physical Review Letters 91 (15), 153003+, quant-ph/0305164.

Nagy, D., J. Asbóth, and P. Domokos (2006a), Acta Physica Hungarica B) Quantum Electronics 26, 141, 10.1556/APH.26.2006.1-2.16.

Nagy, D., J. K. Asboth, P. Domokos, and H. Ritsch (2006b), EPL (Europhysics Letters) 74 (2), 254.

Nagy, D., P. Domokos, A. Vukics, and H. Ritsch (2009), The European Physical Journal D - Atomic, Molecular, Optical and Plasma Physics 55 (3), 659.

Nagy, D., G. Kónya, G. Szirmai, and P. Domokos (2010), Physical Review Letters 104 (13), 130401+.

Nagy, D., G. Szirmai, and P. Domokos (2008), The European Physical Journal D - Atomic, Molecular, Optical and Plasma Physics 48 (1), 127.

Nagy, D., G. Szirmai, and P. Domokos (2011), Physical Review A 84, 043637+.
Nicholson, T., J. Williams, B. Bloom, S. Campbell, M. Martin, M. Swallows, M. Bishof, and J. Ye (2012), Bulletin of the American Physical Society $\mathbf{5 7 .}$

Niedenzu, W., T. Grießer, and H. Ritsch (2011), EPL (Europhysics Letters), 43001+.

Niedenzu, W., R. Schulze, A. Vukics, and H. Ritsch (2010), Physical Review A 82 (4), 043605+.

Nimmrichter, S., K. Hammerer, P. Asenbaum, H. Ritsch, and M. Arndt (2010), New Journal of Physics 12 (8), 083003+.

Nussmann, S., M. Hijlkema, B. Weber, F. Rohde, G. Rempe, and A. Kuhn (2005), Physical Review Letters 95 (17), $173602+$.

Nussmann, S., K. Murr, M. Hijlkema, B. Weber, A. Kuhn, and G. Rempe (2005), Nat Phys, Nature Physics 1 (2), 122 .

Ourjoumtsev, A., A. Kubanek, M. Koch, C. Sames, P. W. H. Pinkse, G. Rempe, and K. Murr (2011), Nature 474 (7353), 623.

Öztop, B., M. Bordyuh, O. E. Müstecaplığlu, and H. E. Türeci (2012), New Journal of Physics 14 (8), 085011+.

Perrin, M., G. L. Lippi, and A. Politi (2001), Physical Review Letters 86, 4520 .

Perrin, M., Z. Ye, and L. M. Narducci (2002), Physical Review A 66, 043809+.

Phillips, W. D. (1998), Reviews of Modern Physics 70 (3), 721.

Pinkse, P., and G. Rempe (2002), "Single Atoms Moving in a High-Finesse Cavity," in Cavity Enhanced Spectroscopies, edited by R. D. van Zee and J. P. Looney, Chap. 13 (Academic Press) pp. 255-295.

Pinkse, P. W. H., T. Fischer, P. Maunz, and G. Rempe (2000), Nature 404 (6776), 365.

Pitaevskii, L., and S. Stringari (2003), $\{B\}$ ose- $\{$ E $\}$ instein $\{C\}$ ondensation, edited by J. Birman, S. F. Friend, C. H. Llewellyn-Smith, M. Rees, D. Sherrington, and G. Veneziano (Oxford University Press, Oxford).

Posch, H., and W. Thirring (2005), Physical Review Letters 95 (25), 251101.

Puppe, T., I. Schuster, A. Grothe, A. Kubanek, K. Murr, P. W. H. Pinkse, and G. Rempe (2007a), Physical Review Letters 99 (1), 013002+.

Puppe, T., I. Schuster, P. Maunz, K. Murr, P. W. H. Pinkse, and G. Rempe (2007b), Journal of Modern Optics 54 (1315), 1927.

Purcell, E. M. (1946), Phys. Rev. 69, 681+.

Purdy, T. P., D. W. C. Brooks, T. Botter, N. Brahms, Z. Y. Ma, and D. M. S. Kurn (2010), Physical Review Letters 105 (13), 133602+.

Raimond, J. M., M. Brune, and S. Haroche (2001), Reviews of Modern Physics 73 (3), 565.

Raizen, M. G., R. J. Thompson, R. J. Brecha, H. J. Kimble, and H. J. Carmichael (1989), Phys. Rev. Lett. 63, 240.

Rangwala, S. A., T. Junglen, T. Rieger, P. W. H. Pinkse, and G. Rempe (2003), Physical Review A 67 (4), 043406+.

Rist, S., C. Menotti, and G. Morigi (2010), Physical Review A 81 (1), 013404+.

Ritsch, H. (2009), Nat Phys 5 (11), 781.

Ritter, S., F. Brennecke, K. Baumann, T. Donner, C. Guerlin, and T. Esslinger (2009), Applied Physics B: Lasers and Optics 95 (2), 213.

Robb, G. R. M., N. Piovella, A. Ferraro, R. Bonifacio, P. W. Courteille, and C. Zimmermann (2004), Physical Review A 69 (4), 041403+. 
Rolston, S. L., and W. D. Phillips (2002), Nature (London) 416, 219.

Romero-Isart, O., M. Juan, R. Quidant, and J. Cirac (2010), New Journal of Physics 12, 033015.

Rzażewski, K., K. Wódkiewicz, and W. Żakowicz (1975), Physical Review Letters 35 (7), 432.

Salzburger, T., P. Domokos, and H. Ritsch (2002), Opt. Express 10 (21), 1204.

Salzburger, T., P. Domokos, and H. Ritsch (2005), Physical Review A 72 (3), 033805+.

Salzburger, T., and H. Ritsch (2004), Physical Review Letters 93 (6), 063002+.

Salzburger, T., and H. Ritsch (2006), Physical Review A 74 (3), 033806+.

Salzburger, T., and H. Ritsch (2007), Physical Review A 75 (6), 061601+.

Salzburger, T., and H. Ritsch (2008), Physical Review A 77, $063620+$.

Salzburger, T., and H. Ritsch (2009), New Journal of Physics 11 (5), 055025+.

Sauer, J. A., K. M. Fortier, M. S. Chang, C. D. Hamley, and M. S. Chapman (2004), Physical Review A 69 (5), 051804+.

Schleier-Smith, M. H., I. D. Leroux, H. Zhang, M. A. Van Camp, and V. Vuletić (2011), Phys. Rev. Lett. 107, 143005.

Schliesser, A., O. Arcizet, R. Rivière, G. Anetsberger, and T. J. Kippenberg (2009), Nat. Phys. 5, 509.

Schulze, R. J., C. Genes, and H. Ritsch (2010), Physical Review A 81 (6), 063820+.

Schuster, I., A. Kubanek, A. Fuhrmanek, T. Puppe, P. W. H. Pinkse, K. Murr, and G. Rempe (2008), Nature Physics $4(5), 382$.

Shore, B. W., and P. L. Knight (1993), Journal of Modern Optics 40 (7), 1195.

Shuman, E. S., J. F. Barry, and D. DeMille (2010), Nature 467 (7317), 820.

Silver, A. O., M. Hohenadler, M. J. Bhaseen, and B. D. Simons (2010), Physical Review A 81, 023617+.

Slama, S., S. Bux, G. Krenz, C. Zimmermann, and P. W. Courteille (2007a), Physical Review Letters 98 (5), $053603+$.

Slama, S., G. Krenz, S. Bux, C. Zimmermann, and P. W. Courteille (2007b), Physical Review A 75 (6), 063620+.

de Souza, A., and C. Tsallis (1997), Physica A: Statistical Mechanics and its Applications 236 (1-2), 52.

Specht, H. P., C. Nolleke, A. Reiserer, M. Uphoff, E. Figueroa, S. Ritter, and G. Rempe (2011), Nature 473 (7346), 190.

Stamper-Kurn, D. M. (2012), "Cavity optomechanics with cold atoms," in Cavity optomechanics, edited by M. Aspelmeyer, T. Kippenberg, and F. Marquardt (Springer) p. (arXiv:1204.4351).

Stenger, J., S. Inouye, A. P. Chikkatur, D. M. S. Kurn, D. E. Pritchard, and W. Ketterle (1999), Physical Review Letters 82 (23), 4569.

Strack, P., and S. Sachdev (2011), Physical Review Letters 107, 277202+.

Strogatz, S. (2000), Physica D: Nonlinear Phenomena 143 (14), 1.

Sun, Q., X. H. Hu, A. C. Ji, and W. M. Liu (2011), Physical Review A 83 (4), 043606+.

Szirmai, G., D. Nagy, and P. Domokos (2009), Physical Review Letters 102 (8), 080401+.

Szirmai, G., D. Nagy, and P. Domokos (2010), Physical Re- view A 81 (4), 043639+.

Tavis, M., and F. W. Cummings (1968), Physical Review Online Archive (Prola) 170 (2), 379.

Teper, I., Y. J. Lin, and V. Vuletić (2006), Physical Review Letters 97 (2), 023002+.

Thompson, J. D., B. M. Zwickl, A. M. Jayich, F. Marquardt, S. M. Girvin, and J. G. E. Harris (2008), \{N\}ature 452 (7183), 72.

Tuchman, A. K., R. Long, G. Vrijsen, J. Boudet, J. Lee, and M. A. Kasevich (2006), PHYSICAL REVIEW A 74 (5), \%7B10.1103/PhysRevA.74.053821\%7D.

Vidal, S. F., G. De Chiara, J. Larson, and G. Morigi (2010), Physical Review A 81 (4), 043407+.

Vidal, S. F., S. Zippilli, and G. Morigi (2007), Physical Review A 76, 053829+.

Vilensky, M. Y., Y. Prior, and I. Sh (2007), Physical Review Letters 99 (10), 103002+.

Vukics, A. (2012), Computer Physics Communications 183 (6), 1381.

Vukics, A., and P. Domokos (2005), Physical Review A 72 (3), 031401+.

Vukics, A., P. Domokos, and H. Ritsch (2004), J. Opt. B: Quantum Semiclass. Opt. 6, 143.

Vukics, A., J. Janszky, and P. Domokos (2005), Journal of Physics B: Atomic, Molecular and Optical Physics 38 (10), 1453.

Vukics, A., C. Maschler, and H. Ritsch (2007), New J. Phys. $9(8), 255+$.

Vukics, A., W. Niedenzu, and H. Ritsch (2009), Physical Review A 79, 013828+.

Vukics, A., and H. Ritsch (2007), The European Physical Journal D - Atomic, Molecular, Optical and Plasma Physics $44(3), 585$.

Vuletic, V. (2001), in Laser physics at the limits, edited by H. Figger, D. Meschede, and C. Zimmermann (Springer, New York) pp. 67-74.

Vuletić, V., H. W. Chan, and A. T. Black (2001), Physical Review A 64 (3), 033405+.

Vuletić, V., and S. Chu (2000), Physical Review Letters $84(17), 3787$.

Walther, H. (2002), Adv. Chem. Phys. 122, 167.

Wang, Y. K., and F. T. Hioe (1973), Physical Review A 7 (3), 831.

Wilk, T., S. C. Webster, A. Kuhn, and G. Rempe (2007a), Science 317 (5837), 488.

Wilk, T., S. C. Webster, H. P. Specht, G. Rempe, and A. Kuhn (2007b), Physical Review Letters 98 (6), 063601+.

Wolke, M., J. Klinner, H. Keßler, and A. Hemmerich (2012), Science 337 (6090), 75.

Xuereb, A., P. Domokos, J. Asbóth, P. Horak, and T. Freegarde (2009), Physical Review A 79 (5), 053810+.

Xuereb, A., T. Freegarde, P. Horak, and P. Domokos (2010), Physical Review Letters 105 (1), 013602+.

Xuereb, A., C. Genes, and A. Dantan (2012), Arxiv preprint arXiv:1202.6210.

Zeppenfeld, M., M. Motsch, P. W. H. Pinkse, and G. Rempe (2009), Physical Review A 80 (4), 041401+.

Zhang, J. M., F. C. Cui, D. L. Zhou, and W. M. Liu (2009), Physical Review A (Atomic, Molecular, and Optical Physics) 79 (3), 033401+.

Zippilli, S., J. Asboth, G. Morigi, and H. Ritsch (2004a), Applied Physics B: Lasers and Optics 79 (8), 969.

Zippilli, S., and G. Morigi (2005), Physical Review Letters 95 (14), 143001+. 
Zippilli, S., and G. Morigi (2007), Physical Review A 72 (5), $053408+$, quant-ph/0508075.

Zippilli, S., G. Morigi, and H. Ritsch (2004b), The Euro- pean Physical Journal D - Atomic, Molecular, Optical and Plasma Physics 31 (3), 507. 